\title{
BORCHERDS PRODUCTS AND ARITHMETIC INTERSECTION THEORY ON HILBERT MODULAR SURFACES
}

\section{JAN H. BRUINIER, JOSÉ I. BURGOS GIL, and ULF KÜHN}

\begin{abstract}
We prove an arithmetic version of a theorem of Hirzebruch and Zagier saying that Hirzebruch-Zagier divisors on a Hilbert modular surface are the coefficients of an elliptic modular form of weight 2. Moreover, we determine the arithmetic selfintersection number of the line bundle of modular forms equipped with its Petersson metric on a regular model of a Hilbert modular surface, and we study Faltings heights of arithmetic Hirzebruch-Zagier divisors.
\end{abstract}

\section{Contents}

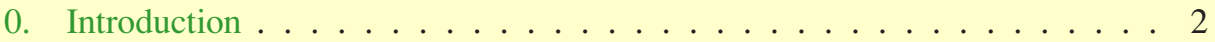

1. Arithmetic Chow rings with pre-log-log forms . . . . . . . . . 7

1.1. Differential forms with growth conditions . . . . . . . . . 8

1.2. Pre-log-log Green objects . . . . . . . . . . . . . 10

1.3. Star products of pre-log-log Green objects . . . . . . . . . . . 14

1.4. Arithmetic Chow rings with pre-log-log forms . . . . . . . . . 17

1.5. Pre-log singular hermitian line bundles and Faltings heights . . . . 19

2. Complex theory of Hilbert modular surfaces . . . . . . . . . . . . 22

2.1. Desingularization and the curve lemma . . . . . . . . . . 23

2.2. Hilbert modular forms and the Petersson metric . . . . . . . . . 26

2.3. Green functions for Hirzebruch-Zagier divisors . . . . . . . . . . . 29

3. Star products on Hilbert modular surfaces . . . . . . . . . . . . . . 38

3.1. Star products for Hirzebruch-Zagier divisors . . . . . . . . . . . . . 38

3.2. Integrals of Green functions . . . . . . . . . . . . . . . . 44

3.3. Star products on isotropic Hirzebruch-Zagier divisors . . . . . . . . . 48

3.4. Star products on Hilbert modular surfaces . . . . . . . . . . 51

4. Borcherds products on Hilbert modular surfaces . . . . . . . . . . . 52

DUKE MATHEMATICAL JOURNAL

Vol. 139, No. 1, (C) 2007

Received 23 November 2005. Revision received 7 July 2006.

2000 Mathematics Subject Classification. Primary 11G18; Secondary 11F41, 14C17, 14G40, 14 C20.

Bruinier partially supported by a Deutsche Forschungsgemeinschaft Heisenberg Fellowship.

Burgos Gil partially supported by Dirección General de Investigación grants BFM2000-0799-c02-01 and BFM2003-02914. 
4.1. Basic properties of Borcherds products . . . . . . . . 52

4.2. Density of Borcherds products . . . . . . . . . . . . . 57

5. Arithmetic theory of Hilbert modular surfaces . . . . . . . . . . . 63

5.1. Moduli spaces of abelian schemes with real multiplication . . . . 63

5.2. Modular morphisms . . . . . . . . . . . . . . . . 67

6. Arithmetic intersection theory on Hilbert modular varieties . . . . . . 75

6.1. Arithmetic Hirzebruch-Zagier divisors . . . . . . . . . . . 76

6.2. Arithmetic intersection numbers and Faltings heights . . . . . . . 78

6.3. $\mathbb{R}$-valued arithmetic intersection theory . . . . . . . . 82

References ........................... 85

\section{Introduction}

It is of special interest in Arakelov theory to determine intrinsic arithmetic intersection numbers of varieties defined over number fields and Faltings heights of its subvarieties. Here, we study Hilbert modular surfaces associated to a real quadratic field $K$. Since these Shimura varieties are noncompact, we consider suitable toroidal compactifications. As the natural metrics of automorphic vector bundles on such varieties have singularities along the boundary (see, e.g., [M], [BKK1]), we work with the extended arithmetic Chow rings constructed in [BKK2].

In their celebrated article [HZ], Hirzebruch and Zagier proved that the generating series for the cohomology classes of Hirzebruch-Zagier divisors is a holomorphic modular form of weight 2. A different proof was obtained by Borcherds [B2]. Moreover, it was shown by Franke $[\mathrm{F}]$ and by Hausmann [Ha] that the product of this generating series with the first Chern class of the line bundle of modular forms (i.e., the generating series for the hyperbolic volumes) equals a particular holomorphic Eisenstein series $E(\tau)$ of weight 2 .

In the present article, we define certain arithmetic Hirzebruch-Zagier divisors on a suitable regular model of the Hilbert modular surface. We show that the generating series of their classes in the arithmetic Chow ring is a holomorphic modular form (of the same level, weight, and character as in the case of Hirzebruch and Zagier). The main result of our work is that the product of this generating series with the square of the first arithmetic Chern class of the line bundle of modular forms equipped with its Petersson metric is equal to a multiple of $E(\tau)$. The factor of proportionality is the arithmetic self-intersection number of the line bundle of modular forms which is computed explicitly.

Since Hilbert modular surfaces can also be viewed as Shimura varieties associated to the orthogonal group of a rational quadratic space of signature $(2,2)$, these results are related to the program described by Kudla in [Ku2], [Ku3], [Ku4], [Ku6] (for a discussion of results in that direction, see also the references therein). However, notice that there are several technical differences. For instance, in the present case, 
the Shimura variety has to be compactified, the arithmetic Hirzebruch-Zagier divisors contain boundary components, and we work with different Green functions.

We show that the arithmetic self-intersection number of the line bundle of modular forms is essentially given by the logarithmic derivative at $s=-1$ of the Dedekind zeta function $\zeta_{K}(s)$ of $K$. It equals the expression conjectured in [Kü1]. We refer to Theorem B for the precise statement.

We also determine the Faltings heights of those Hirzebruch-Zagier divisors that are disjoint to the boundary. It is well known that their normalizations are isomorphic to compact Shimura curves associated with quaternion algebras. This result will be used in a subsequent work [KK] to determine the arithmetic self-intersection number of the Hodge bundle equipped with the hyperbolic metric on Shimura curves.

Our formulas provide further evidence for the conjecture of Kramer, based on results obtained in $[\mathrm{K}]$ and [Kü1], that the arithmetic volume is essentially the derivative of the zeta value for the volume of the fundamental domain, the conjecture of Kudla on the constant term of the derivative of certain Eisenstein series (see [Ku3], [Ku4], [Ku6], [KRY]), and the conjecture of Maillot and Roessler on special values of logarithmic derivatives of Artin $L$-functions (see [MR1], [MR2]).

Our approach requires various results on regular models for Hilbert modular surfaces (see $[\mathrm{R}],[\mathrm{DP}],[\mathrm{P}]$ ), as well as an extensive use of the theory of Borcherds products (see [B1], [B2], [Br1], [Br2]). Another central point is the $q$-expansion principle (see $[\mathrm{R}],[\mathrm{C}]$ ), which relates analysis to geometry. Finally, a result on Galois representations (see $[\mathrm{OS}]$ ) allows us to replace delicate calculations of finite intersection numbers with density results for Borcherds products.

We now describe the content of this article in more detail.

Since the natural metrics on automorphic line bundles have singularities along the boundary, we cannot use arithmetic intersection theory as presented in $[\mathrm{SABK}]$ and have to work with the extension developed in [BKK2]. In particular, our arithmetic cycles are classes in the generalized arithmetic Chow ring $\widehat{\mathrm{CH}}^{*}(\mathscr{X}, \mathscr{D}$ pre $)$, in which the differential forms are allowed to have certain log-log singularities along a fixed normal crossing divisor. We recall some of its basic properties in Section 1. Choosing representatives, the arithmetic intersection product of two arithmetic cycles can be split into the sum of a geometric contribution as in [SABK] and an integral over a star product of Green objects. The formulas for the latter quantity generalize those of $[\mathrm{SABK}]$ and may contain additional boundary terms.

We concentrate on the analytic aspects in Sections 2 and 3. The geometric contribution is considered in Sections 5 and 6.

We begin Section 2 by recalling some facts on the analytic theory of Hilbert modular surfaces. Throughout the article, the discriminant $D$ of $K$ is assumed to be prime. Let $\Gamma$ be a subgroup of finite index of the Hilbert modular group $\Gamma_{K}=\mathrm{SL}_{2}\left(\mathcal{O}_{K}\right)$, where $\mathcal{O}_{K}$ denotes the ring of integers in $K$. We consider an (at the outset, arbitrary) 
desingularization $\tilde{X}(\Gamma)$ of the Baily-Borel compactification $X(\Gamma)$ of $\Gamma \backslash \mathbb{H}^{2}$, where $\mathbb{H}$ is the upper complex half-plane. Here, the "curve lemma" due to Freitag [Fr1, Satz 1, Hilfssatz 2] is a main technical tool to obtain adequate local descriptions. We introduce the line bundle of modular forms $\mathscr{M}_{k}(\Gamma)$ of weight $k$ with its Petersson metric $\|\cdot\|_{\text {Pet }}$, which is singular along the normal crossing divisor formed by the exceptional curves of the desingularization. We show that the Petersson metric is a pre-log singular hermitian metric in the sense of [BKK2].

For any positive integer $m$, there is an algebraic divisor $T(m)$ on $X(\Gamma)$ called the Hirzebruch-Zagier divisor of discriminant $m$. These divisors play a central role in the study of the geometry of Hilbert modular surfaces (see, e.g., [G]). We consider the Green function $\Phi_{m}\left(z_{1}, z_{2}, s\right)$ on the quasi-projective variety $\Gamma \backslash \mathbb{H}^{2}$, associated to $T(m)$, introduced in [Br1]. A new point here is the explicit description of the constant term, given in Theorem 2.11, which involves $\zeta_{K}(s)$ and a certain generalized divisor function $\sigma_{m}(s)$ (see (2.32)). We then define the automorphic Green function $G_{m}$ for $T(m)$ to be the constant term in the Laurent expansion at $s=1$ of $\Phi_{m}\left(z_{1}, z_{2}, s\right)$ minus a natural normalizing constant, which is needed to obtain compatibility with the theory of Borcherds products as used in Section 4. By abuse of notation, we also denote by $T(m)$ the pullback of $T(m)$ to $\widetilde{X}(\Gamma)$. By means of the curve lemma, we show that the Green function $G_{m}$ defines a pre-log-log Green object in the sense of [BKK2] for the divisor $T(m)$ in the projective variety $\widetilde{X}(\Gamma)$.

In Section 3, we specialize the general formula for the star product to the Green objects $\mathfrak{g}\left(m_{j}\right)=\left(-2 \partial \bar{\partial} G_{m_{j}}, G_{m_{j}}\right)$ associated to suitable triples of Hirzebruch-Zagier divisors $T\left(m_{j}\right)$ (see Theorem 3.3). In this case, using the curve lemma, it can be shown that certain boundary terms vanish. As a consequence, the resulting formula depends only on the Baily-Borel compactification $X(\Gamma)$ and is independent of the choices made in the desingularization. An analogous formula holds for the star product associated to a Hirzebruch-Zagier divisor $T(m)$ and the divisors of two Hilbert modular forms. In that way, we reduce the analytical contributions to the arithmetic intersection numbers in question to integrals of Green functions $G_{m}$ over $X(\Gamma)$ and star products on Hirzebruch-Zagier divisors $T(m)$.

The analysis of Section 2 allows us to determine the integral of $G_{m}$ in terms of the logarithmic derivatives of $\zeta_{K}(s)$ and $\sigma_{m}(s)$ at $s=-1$ (Corollary 3.9). If $p$ is a prime that splits in $K$, then $T(p)$ is birational to the modular curve $X_{0}(p)$. We determine the star products on these $T(p)$ using the results of [Kü2] in Theorem 3.13.

The proofs of our main results rely on the theory of Borcherds products in a vital way. Therefore, in Section 4, we recall some of their basic properties with an emphasis on the construction given in [Br1]. Borcherds products on Hilbert modular surfaces are particular meromorphic Hilbert modular forms that arise as regularized theta lifts of certain weakly holomorphic elliptic modular forms of weight zero. They enjoy striking arithmetic properties. For instance, a sufficiently large power of any 
holomorphic Borcherds product has coprime integral Fourier coefficients. Hence, by the $q$-expansion principle, it defines a section of the line bundle of modular forms over $\mathbb{Z}$. Moreover, the divisors of Borcherds products are explicit linear combinations of Hirzebruch-Zagier divisors, dictated by the poles of the input modular form. By [Br1], the logarithm of their Petersson norm is precisely given by a linear combination of the Green functions $G_{m}$.

By a careful analysis of the obstruction space for the existence of Borcherds products, we prove that for any given linear combination of Hirzebruch-Zagier divisors $C$, there are infinitely many Borcherds products $F_{1}, F_{2}$ such that $C \cap \operatorname{div}\left(F_{1}\right) \cap$ $\operatorname{div}\left(F_{2}\right)=\emptyset$ and such that further technical conditions are satisfied (Theorem 4.12). This gives an ample supply of sections of the line bundle of modular forms for which the associated star products can be calculated. In the rest of this section, we essentially show that the subspace of $\operatorname{Pic}\left(X\left(\Gamma_{K}\right)\right) \otimes_{\mathbb{Z}} \mathbb{Q}$, spanned by all HirzebruchZagier divisors, is already generated by the $T(p)$, where $p$ is a prime that splits in $K$ (Corollary 4.16). This fact can be viewed as an explicit moving lemma for HirzebruchZagier divisors (see Theorem 6.1), and it is crucial in the proof of Theorem B since it noticeably simplifies the calculations at finite places.

In Section 5, we recall the arithmetic theory of Hilbert modular surfaces. Unfortunately, there are currently no references for projective regular models defined over Spec $\mathbb{Z}$. Therefore we work with regular models over the subring $\mathbb{Z}\left[\zeta_{N}, 1 / N\right]$ of the $N$ th cyclotomic field $\mathbb{Q}\left(\zeta_{N}\right)$. More precisely, we consider toroidal compactifications $\widetilde{\mathscr{H}}(N)$ of the moduli scheme associated with the Hilbert modular variety for the principal congruence subgroup $\Gamma_{K}(N)$ of arbitrary level $N \geq 3$. We define $\mathscr{T}_{N}(m) \subseteq \widetilde{\mathscr{H}}(N)$ as the Zariski closure of the Hirzebruch-Zagier divisor $T(m)$ on the generic fiber. This definition is well behaved with respect to pullbacks and is compatible with the theory of Borcherds products (see Proposition 5.7). If $p$ is a split prime, there exists a natural modular morphism from the compactified moduli space of elliptic curves with a subgroup of order $p$ to the minimal compactification of $\mathscr{H}(1)$ whose image is essentially $\mathscr{T}_{1}(p)$ (see Proposition 5.13). This allows us to determine the geometric contribution of the arithmetic intersection numbers in question using the projection formula (Proposition 5.15).

In Section 6, the arithmetic intersection theory of Hilbert modular varieties is studied. There exists no arithmetic intersection theory for the stack $\widetilde{\mathscr{H}}(1)$. Thus, following a suggestion of S. S. Kudla, we work with the tower of schemes $\{\widetilde{\mathscr{H}}(N)\}_{N \geq 3}$ as a substitute for $\widetilde{\mathscr{H}}(1)$. We define the arithmetic Chow ring for the Hilbert modular variety to be the inverse limit of the arithmetic Chow rings associated to this tower. In this way, we obtain $\mathbb{R}$-valued arithmetic intersection numbers; even so, for every $N$, we need to calculate the arithmetic intersection numbers only up to contributions at the finite places dividing the level. 
We introduce the arithmetic Hirzebruch-Zagier divisors $\widehat{\mathscr{T}}_{N}(m)=$ $\left(\mathscr{T}_{N}(m), \mathfrak{g}_{N}(m)\right)$, where $\mathfrak{g}_{N}(m)$ is the pullback of the automorphic Green object described in Section 2. The subgroup of the first arithmetic Chow group generated by these divisors has finite rank (see Theorem 6.1). We let $\widehat{\mathrm{c}}_{1}\left(\overline{\mathscr{M}}_{k}\left(\Gamma_{K}(N)\right)\right)$ be the first arithmetic Chern class of the pre-log singular hermitian line bundle $\overline{\mathscr{M}}_{k}\left(\Gamma_{K}(N)\right)$ of modular forms of weight $k$ with the Petersson metric.

The sequences $\widehat{\mathscr{T}}(m):=\left(\widehat{\mathscr{T}}_{N}(m)\right)_{N \geq 3}$ and $\widehat{\mathrm{c}}_{1}\left(\overline{\mathscr{M}}_{k}\right):=\left(\widehat{\mathrm{c}}_{1}\left(\overline{\mathscr{M}}_{k}\left(\Gamma_{K}(N)\right)\right)\right)_{N \geq 3}$ define classes in the arithmetic Chow group $\widehat{\mathrm{CH}}^{1}\left(\widetilde{\mathscr{H}}, \mathscr{D}_{\text {pre }}\right)$, which is defined as the inverse limit of the arithmetic Chow groups $\widehat{\mathrm{CH}}^{1}\left(\widetilde{\mathscr{H}}(N), \mathscr{D}_{\text {pre }}\right)$.

Let $M_{2}^{+}\left(D, \chi_{D}\right)$ be the space of holomorphic modular forms of weight 2 for the congruence subgroup $\Gamma_{0}(D) \subseteq \mathrm{SL}_{2}(\mathbb{Z})$ with character $\chi_{D}=\left(\frac{D}{.}\right)$ satisfying the "plus-condition" as in [HZ]. It has a natural $\mathbb{Q}$-structure that is given by modular forms with rational Fourier coefficients.

\section{THEOREM A}

The arithmetic generating series

$$
\widehat{A}(\tau)=\widehat{\mathrm{c}}_{1}\left(\overline{\mathscr{M}}_{1 / 2}^{\vee}\right)+\sum_{m>0} \widehat{\mathscr{T}}(m) q^{m}
$$

is a holomorphic modular form in $M_{2}^{+}\left(D, \chi_{D}\right)$ with values in $\widehat{\mathrm{CH}}^{1}\left(\widetilde{\mathscr{H}}, \mathscr{D}_{\mathrm{pre}}\right)_{\mathbb{Q}}$, that is, an element of $M_{2}^{+}\left(D, \chi_{D}\right) \otimes_{\mathbb{Q}} \widehat{\mathrm{CH}}^{1}\left(\widetilde{\mathscr{H}}, \mathscr{D}_{\mathrm{pre}}\right)_{\mathbb{Q}}$.

To establish Theorem A, we show that the Green functions $G_{m}$ are compatible with the relations in the arithmetic Chow group given by Borcherds products (Theorem 4.3). Moreover, using the $q$-expansion principle and the results of Section 4, we prove that the divisor of a Borcherds product over $\mathbb{Z}$ is horizontal and therefore compatible with the definition of the Hirzebruch-Zagier divisors over $\mathbb{Z}$ as Zariski closures (Proposition 5.7). Now, modularity follows by a duality argument due to Borcherds in the geometric situation (see [B2]).

Our main result below provides some information on the position of the generating series in the arithmetic Chow group. Its proof is much more involved since, in contrast to Theorem A, it requires the computation of arithmetic intersections.

\section{THEOREM B}

We have the following identities of arithmetic intersection numbers:

$$
\widehat{A}(\tau) \cdot \widehat{\mathrm{c}}_{1}\left(\overline{\mathscr{M}}_{k}\right)^{2}=\frac{k^{2}}{2} \zeta_{K}(-1)\left(\frac{\zeta_{K}^{\prime}(-1)}{\zeta_{K}(-1)}+\frac{\zeta^{\prime}(-1)}{\zeta(-1)}+\frac{3}{2}+\frac{1}{2} \log (D)\right) \cdot E(\tau) .
$$


Here, E( $\tau)$ denotes the Eisenstein series defined in (4.2). In particular, the arithmetic self-intersection number of $\overline{\mathscr{M}}_{k}$ is given by

$$
\overline{\mathscr{M}}_{k}^{3}=-k^{3} \zeta_{K}(-1)\left(\frac{\zeta_{K}^{\prime}(-1)}{\zeta_{K}(-1)}+\frac{\zeta^{\prime}(-1)}{\zeta(-1)}+\frac{3}{2}+\frac{1}{2} \log (D)\right) .
$$

\section{THEOREM C}

If $T(m)$ is a Hirzebruch-Zagier divisor that is disjoint to the boundary, then the Faltings height of its model $\mathscr{T}(m)$ with respect to $\overline{\mathscr{M}}_{k}$ equals

$$
\mathrm{ht}_{\overline{\mathscr{M}}_{k}}(\mathscr{T}(m))=-(2 k)^{2} \operatorname{vol}(T(m))\left(\frac{\zeta^{\prime}(-1)}{\zeta(-1)}+\frac{1}{2}+\frac{1}{2} \frac{\sigma_{m}^{\prime}(-1)}{\sigma_{m}(-1)}\right) .
$$

The arithmetic intersection theory used here can be viewed as a substitute of an arithmetic intersection theory on stacks for our particular situation. Notice that any reasonable arithmetic intersection theory for the coarse moduli space without level structure has to map into the above theory so that all arithmetic degrees and heights would agree.

Many arguments of the present article can (probably) be generalized to Shimura varieties of type $\mathrm{O}(2, n)$, where one would like to study the arithmetic intersection theory of Heegner divisors (also referred to as special divisors; see, e.g., [Ku6], [Ku1]). In fact, the work on this article already motivated generalizations of partial aspects. For instance, automorphic Green functions are investigated in [BK] and the curve lemma in [Br3]. However, progress on the whole picture will (in the near future) be limited to the exceptional cases, where regular models of such Shimura varieties are available.

\section{Arithmetic Chow rings with pre-log-log forms}

The natural metrics that appear, when considering automorphic vector bundles on noncompact locally symmetric spaces, do not extend to smooth metrics on a compactification of the space. This is the case, for instance, for the Petersson metric on the line bundle of modular forms on a Hilbert modular surface. Therefore the arithmetic intersection theory developed by Gillet and Soulé [GS1], [GS2] cannot be applied directly to study this kind of hermitian vector bundles. In the articles [BKK1] and [BKK2], there are two extensions of the arithmetic intersection theory which are suited to the study of automorphic vector bundles. The first of these theories is based on what are called pre-log and pre-log-log forms, and the second extension is based in $\log$ and $\log -\log$ forms. The difference between them is that a differential form $\omega$ is pre-log or pre-log-log if $\omega, \partial \omega, \bar{\partial} \omega$, and $\partial \bar{\partial} \omega$ satisfy certain growth conditions, whereas a differential form is called log or log-log form if all the derivatives of all the components of the differential form satisfy certain growth conditions. The main 
advantage of the second definition is that we know the cohomology computed by the log and log-log forms; therefore the arithmetic Chow groups defined with them have better properties. By contrast, the main advantage of the first approach is that it is easier to check that a particular form satisfies the conditions defining pre-log and pre-log-log forms. Since the arithmetic intersection products obtained by both theories are compatible, for simplicity we have chosen to use in this article the first of these theories.

\subsection{Differential forms with growth conditions}

\section{Notation 1.1}

Let $X$ be a complex algebraic manifold of dimension $d$, and let $D$ be a normal crossing divisor of $X$. We denote by $\mathscr{E}_{X}^{*}$ the sheaf of smooth complex differential forms on $X$. Moreover, we write $U=X \backslash D$, and we let $j: U \rightarrow X$ be the inclusion.

Let $V$ be an open coordinate subset of $X$ with coordinates $z_{1}, \ldots, z_{d}$; we put $r_{i}=\left|z_{i}\right|$. We say that $V$ is adapted to $D$ if the divisor $D$ is locally given by the equation $z_{1} \cdots z_{k}=0$. We assume that the coordinate neighborhood $V$ is small enough; more precisely, we assume that all the coordinates satisfy $r_{i}<1 / e^{e}$, which implies that $\log 1 / r_{i}>e$ and $\log \left(\log 1 / r_{i}\right)>1$.

If $f$ and $g$ are two complex functions, we write $f \prec g$ if there exists a constant $C>0$ such that $|f(x)| \leq C|g(x)|$ for all $x$ in the domain of definition under consideration.

\section{Definition 1.2}

We say that a smooth complex function $f$ on $X \backslash D$ has $\log$-log growth along $D$ if we have

$$
f\left(z_{1}, \ldots, z_{d}\right) \prec \prod_{i=1}^{k} \log \left(\log \frac{1}{r_{i}}\right)^{M}
$$

for any coordinate subset $V$ adapted to $D$ and some positive integer $M$. The sheaf of differential forms on $X$ with log-log growth along $D$ is the subalgebra of $j_{*} \mathscr{E}_{U}^{*}$ generated, in each coordinate neighborhood $V$ adapted to $D$, by the functions with $\log -\log$ growth along $D$ and the differentials

$$
\begin{array}{ll}
\frac{\mathrm{d} z_{i}}{z_{i} \log \left(1 / r_{i}\right)}, \frac{\mathrm{d} \bar{z}_{i}}{\bar{z}_{i} \log \left(1 / r_{i}\right)}, & \text { for } i=1, \ldots, k, \\
\mathrm{~d} z_{i}, \mathrm{~d} \bar{z}_{i}, & \text { for } i=k+1, \ldots, d .
\end{array}
$$

A differential form with log-log growth along $D$ is called a log-log growth form. 


\section{Definition 1.3}

A $\log -\log$ growth form $\omega$ such that $\partial \omega, \bar{\partial} \omega$, and $\partial \bar{\partial} \omega$ are also $\log -\log$ growth forms is called a pre-log-log form. The sheaf of pre-log-log forms is the subalgebra of $j_{*} \mathscr{E}_{U}^{*}$ generated by the pre-log-log forms. We denote this complex by $\mathscr{E}_{X}^{*}\langle\langle D\rangle\rangle_{\text {pre }}$.

In [BKK2, Proposition 7.6], it is shown that pre-log-log forms are integrable and the currents associated to them do not have residues.

The sheaf $\mathscr{E}_{X}^{*}\langle\langle D\rangle\rangle_{\text {pre }}$, together with its real structure, its bigrading, and the usual differential operators $\partial, \bar{\partial}$, is easily checked to be a sheaf of Dolbeault algebras. We call it the Dolbeault algebra of pre-log-log forms. Observe that it is the maximal subsheaf of Dolbeault algebras of the sheaf of differential forms with log-log growth.

\section{Definition 1.4}

We say that a smooth complex function $f$ on $U$ has $\log$ growth along $D$ if we have

$$
f\left(z_{1}, \ldots, z_{d}\right) \prec \prod_{i=1}^{k} \log \left(\frac{1}{r_{i}}\right)^{M}
$$

for any coordinate subset $V$ adapted to $D$ and some positive integer $M$. The sheaf of differential forms on $X$ with log growth along $D$ is the subalgebra of $j_{*} \mathscr{E}_{U}^{*}$ generated, in each coordinate neighborhood $V$ adapted to $D$, by the functions with $\log$ growth along $D$ and the differentials

$$
\begin{aligned}
& \frac{\mathrm{d} z_{i}}{z_{i}}, \frac{\mathrm{d} \bar{z}_{i}}{\bar{z}_{i}}, \quad \text { for } i=1, \ldots, k, \\
& \mathrm{~d} z_{i}, \mathrm{~d} \bar{z}_{i}, \quad \text { for } i=k+1, \ldots, d \text {. }
\end{aligned}
$$

A differential form with $\log$ growth along $D$ is called a $\log$ growth form.

\section{Definition 1.5}

A $\log$ growth form $\omega$ such that $\partial \omega, \bar{\partial} \omega$, and $\partial \bar{\partial} \omega$ are also log growth forms is called a pre-log form. The sheaf of pre-log forms is the subalgebra of $j_{*} \mathscr{E}_{U}^{*}$ generated by the pre-log forms. We denote this complex by $\mathscr{E}_{X}^{*}\langle D\rangle_{\text {pre }}$.

The sheaf $\mathscr{E}_{X}^{*}\langle D\rangle_{\text {pre }}$, together with its real structure, its bigrading, and the usual differential operators $\partial, \bar{\partial}$, is easily checked to be a sheaf of Dolbeault algebras. We call it the Dolbeault algebra of pre-log forms. It is the maximal subsheaf of Dolbeault algebras of the sheaf of differential forms with log growth.

For the general situation of interest to us, we need a combination of the concepts of pre-log-log and pre-log forms. 


\section{Notation 1.6}

Let $X, D, U$, and $j$ be as above. Let $D_{1}$ and $D_{2}$ be normal crossing divisors, which may have common components, and such that $D=D_{1} \cup D_{2}$. We denote by $D_{2}^{\prime}$ the union of the components of $D_{2}$ which are not contained in $D_{1}$. We say that the open coordinate subset $V$ is adapted to $D_{1}$ and $D_{2}$, if $D_{1}$ has the equation $z_{1} \cdots z_{k}=0, D_{2}^{\prime}$ has the equation $z_{k+1} \cdots z_{l}=0$, and $r_{i}=\left|z_{i}\right|<1 / e^{e}$ for $i=1, \ldots, d$.

\section{Definition 1.7}

We define the sheaf of differential forms with log growth along $D_{1}$ and log-log growth along $D_{2}$ to be the subalgebra of $j_{*} \mathscr{E}_{U}^{*}$ generated by differential forms with log growth along $D_{1}$ and $\log$-log growth along $D_{2}$.

A differential form with $\log$ growth along $D_{1}$ and $\log$-log growth along $D_{2}$ is called a mixed growth form if the divisors $D_{1}$ and $D_{2}$ are clear from the context.

\section{Definition 1.8}

Let $X, D=D_{1} \cup D_{2}, U$, and $j$ be as before. A mixed growth form $\omega$ such that $\partial \omega, \bar{\partial} \omega$, and $\partial \bar{\partial} \omega$ are also mixed growth forms is called a mixed form. The sheaf of mixed forms is the subalgebra of $j_{*} \mathscr{E}_{U}^{*}$ generated by the mixed forms. We denote this complex by $\mathscr{E}_{X}^{*}\left\langle D_{1}\left\langle D_{2}\right\rangle\right\rangle_{\text {pre }}$.

The sheaf $\mathscr{E}_{X}^{*}\left\langle D_{1}\left\langle D_{2}\right\rangle\right\rangle_{\text {pre }}$, together with its real structure, its bigrading, and the usual differential operators $\partial, \bar{\partial}$, is easily checked to be a sheaf of Dolbeault algebras. We call it the Dolbeault algebra of mixed forms. Observe that we have, by definition,

$$
\mathscr{E}_{X}^{*}\left\langle D_{1}\left\langle D_{2}\right\rangle\right\rangle_{\mathrm{pre}}=\mathscr{E}_{X}^{*}\left\langle D_{1}\left\langle D_{2}^{\prime}\right\rangle\right\rangle_{\mathrm{pre}} .
$$

\subsection{Pre-log-log Green objects}

\section{Notation 1.9}

Let $X$ be a complex algebraic manifold of dimension $d$, and let $D$ be a normal crossing divisor. We denote by $\underline{X}$ the pair $(X, D)$. If $W \subseteq X$ is an open subset, we write $\underline{W}=(W, D \cap W)$.

In the sequel, we consider all operations adapted to the pair $\underline{X}$. For instance, if $Y \subsetneq X$ is a closed algebraic subset and $W=X \backslash Y$, then an embedded resolution of singularities of $Y$ in $\underline{X}$ is a proper modification $\pi: \widetilde{X} \rightarrow X$ such that $\left.\pi\right|_{\pi^{-1}(W)}$ : $\pi^{-1}(W) \rightarrow W$ is an isomorphism, and

$$
\pi^{-1}(Y), \quad \pi^{-1}(D), \quad \pi^{-1}(Y \cup D)
$$

are normal crossing divisors on $\widetilde{X}$. Using Hironaka's theorem on the resolution of singularities (see [Hi]), one can see that such an embedded resolution of singularities exists. 
Analogously, a normal crossing compactification of $\underline{X}$ is a smooth compactification $\bar{X}$ such that the closure $\bar{D}$ of $D$, the subset $B_{\bar{X}}=\bar{X} \backslash X$, and the subset $B_{\bar{X}} \cup \bar{D}$ are normal crossing divisors.

Given a diagram of normal crossing compactifications of $\underline{X}$,

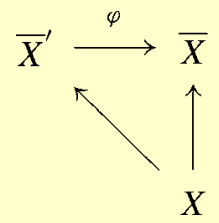

with divisors $B_{\bar{X}^{\prime}}$ and $B_{\bar{X}}$ at infinity, respectively, then by functoriality of mixed forms, there is an induced morphism

$$
\varphi^{*}: \mathscr{E}_{\bar{X}}^{*}\left\langle B_{\bar{X}}\langle\bar{D}\rangle\right\rangle_{\mathrm{pre}} \longrightarrow \mathscr{E}_{\bar{X}^{\prime}}^{*}\left\langle B_{\bar{X}^{\prime}}\left\langle\bar{D}^{\prime}\right\rangle\right\rangle_{\mathrm{pre}} .
$$

In order to have a complex that is independent of the choice of a particular compactification, we take the limit over all possible compactifications. Namely, we denote

$$
E_{\mathrm{pre}}^{*}(\underline{X})^{\circ}=\lim _{\longrightarrow} \Gamma\left(\bar{X}, \mathscr{E} \bar{X} *\left(B_{\bar{X}}\langle\bar{D}\rangle\right\rangle_{\mathrm{pre}}\right),
$$

where the limit is taken over all normal crossing compactifications $\bar{X}$ of $\underline{X}$.

The assignment that sends an open subset $U$ of $X$ to $E_{\text {pre }}^{*}(U)$ is a totally acyclic sheaf in the Zariski topology (see [BKK1, Remark $\left.3.8^{\prime}\right]$ ); we denote it by $E_{\mathrm{pre}, \underline{X}}^{*}$.

\section{Definition 1.10}

Let $\underline{X}=(X, D)$ be as above. Then we define the complex $E_{\mathrm{pre}}^{*}(\underline{X})$ of differential forms on $X$, pre-log along infinity, and pre-log-log along $D$ as the complex of global sections of $E_{\mathrm{pre}, \underline{X}}^{*}$, that is,

$$
E_{\mathrm{pre}}^{*}(\underline{X})=\Gamma\left(X, E_{\mathrm{pre}, \underline{X}}^{*}\right) .
$$

Notation 1.11 (see [BKK2, Section 5.2])

Let $X$ be a smooth real variety, and let $D$ be a normal crossing divisor defined over $\mathbb{R}$; as before, we write $\underline{X}=(X, D)$. For any $U \subseteq X$, the complex $E_{\text {pre }}^{*}(\underline{U})$ is a Dolbeault algebra with respect to the wedge product. For any Zariski open subset $U \subseteq X$, we put

$$
\mathscr{D}_{\mathrm{pre}, \underline{X}}^{*}(U, *)=\left(\mathscr{D}_{\mathrm{pre}, \underline{X}}^{*}(U, *), \mathrm{d}_{\mathscr{D}_{\mathrm{pre}}}\right)=\left(\mathscr{D}^{*}\left(E_{\mathrm{pre}}\left(\underline{U}_{\mathbb{C}}\right), *\right)^{\sigma}, \mathrm{d}_{\mathscr{D}_{\mathrm{pre}}}\right),
$$


where $\mathscr{D}^{*}\left(E_{\mathrm{pre}}\left(\underline{U}_{\mathbb{C}}\right), *\right)$ is the Deligne algebra associated to the Dolbeault algebra $E_{\mathrm{pre}}^{*}(\underline{U})$ and $\sigma$ is the antilinear involution $\omega \mapsto \overline{F_{\infty}(\omega)}$ (see [BKK2, Definition 7.18]). When $\underline{X}=(X, D)$ is clear from the context, we write $\mathscr{D}_{\text {pre }}^{*}(U, *)$ instead of $\mathscr{D}_{\text {pre }, \underline{X}}^{*}(U, *)$.

The arithmetic complex $\mathscr{D}_{\text {pre }}$ made out of pre-log and pre-log-log forms can be seen as the complex that satisfies the minimal requirement needed to allow log-log singularities along a fixed divisor with normal crossing as well as to have a theory of arithmetic intersection numbers. Observe that such singularities naturally occur if one works with automorphic vector bundles (see $[\mathrm{M}]$ ).

Let $U \rightarrow X$ be an open immersion, and let $Y=X \backslash U$. For integers $n, p$, we write

$$
\widehat{H}_{\mathscr{D}_{\mathrm{pre}}, Y}^{n}(X, p)=\widehat{H}^{n}\left(\mathscr{D}_{\mathrm{pre}, \underline{X}}^{*}(X, p), \mathscr{D}_{\mathrm{pre}, \underline{X}}^{*}(U, p)\right),
$$

where the latter groups are truncated relative cohomology groups (see [BKK2, Definition 2.55]). Recall that a class $\mathfrak{g} \in \widehat{H}_{\mathscr{D}_{\mathrm{pre}}, Y}^{n}(X, p)$ is represented by a pair $\mathfrak{g}=(\omega, \widetilde{g})$, with $\omega \in \mathrm{Z}\left(\mathscr{D}_{\text {pre }}^{n}(X, p)\right)$ a cocycle and $\widetilde{g} \in \widetilde{\mathscr{D}}_{\text {pre }}^{n-1}(U, p):=\mathscr{D}_{\text {pre }}^{n-1}(U, p) / \operatorname{Imd}_{\mathscr{D}_{\text {pre }}}$, such that $\mathrm{d}_{\mathscr{D}_{\text {pre }}} \tilde{g}=\omega$. There are morphisms

$$
\omega: \widehat{H}_{\mathscr{D}_{\mathrm{pre}}, Y}^{n}(X, p) \longrightarrow \mathrm{Z}\left(\mathscr{D}_{\mathrm{pre}}^{n}(X, p)\right),
$$

given by $\omega(\mathfrak{g})=\omega(\omega, \widetilde{g})=\omega$, and surjective morphisms

$$
\mathrm{cl}: \widehat{H}_{\mathscr{D}_{\mathrm{pre}}, Y}^{n}(X, p) \longrightarrow H_{\mathscr{D}_{\mathrm{pre}}, Y}^{n}(X, p),
$$

given by sending the class of the pair $(\omega, \widetilde{g})$ in $\widehat{H}_{\mathscr{D}_{\mathrm{pre}}, Y}^{n}(X, p)$ to its class $[\omega, \widetilde{g}]$ in the cohomology group $H^{n}\left(\mathscr{D}_{\text {pre }}^{*}(X, p), \mathscr{D}_{\text {pre }}^{*}(U, p)\right)$.

\section{Definition 1.12}

Let $y$ be a $p$-codimensional algebraic cycle on $X$ with supp $y \subseteq Y$. A weak pre-log$\log$ Green object for $y$ (with support in $Y$ ) is an element $\mathfrak{g}_{y} \in \widehat{H}_{\mathscr{D}_{\text {pre }}, Y}^{2 p}(X, p)$ such that

$$
\operatorname{cl}\left(\mathfrak{g}_{y}\right)=\operatorname{cl}(y) \in H_{\mathscr{D}_{\mathrm{pr}}, Y}^{2 p}(X, p)
$$

here, the class $\operatorname{cl}(y)$ is given by the image of the class of the cycle $y$ in real DeligneBeilinson cohomology via the natural morphism $H_{\mathscr{D}, Y}^{2 p}(X, \mathbb{R}(p)) \rightarrow H_{\mathscr{D}_{\mathrm{pre}}, Y}^{2 p}(X, p)$. If $Y=\operatorname{supp} y$, then $\mathfrak{g}_{y}$ is called a pre-log-log Green object for $y$.

The surjectivity of the morphism cl implies that any algebraic cycle as before has a weak pre-log-log Green object with support in $Y$. For the convenience of the reader, 
we now recall that

$$
\mathrm{Z}\left(\mathscr{D}_{\mathrm{pre}}^{2 p}(X, p)\right)=\left\{\omega \in E_{\mathrm{pre}}^{p, p}(\underline{X}) \cap E_{\mathrm{pre}, \mathbb{R}}^{2 p}(\underline{X}, p) \mid \mathrm{d} \omega=0\right\}
$$

and

$$
\widetilde{\mathscr{D}}_{\text {pre }}^{2 p-1}(U, p)=\left\{g \in E_{\mathrm{pre}}^{p-1, p-1}(\underline{U}) \cap E_{\mathrm{pre}, \mathbb{R}}^{2 p-2}(\underline{U}, p-1)\right\} /(\operatorname{Im} \partial+\operatorname{Im} \bar{\partial}),
$$

where $\mathrm{d}=\partial+\bar{\partial}$ and $\partial$, respectively, $\bar{\partial}$, are the usual holomorphic, respectively, antiholomorphic, derivatives. Then a weak pre-log-log Green object for $y$, as above, is represented by a pair

$$
\left(-2 \partial \bar{\partial} g_{y}, \widetilde{g}_{y}\right) \in \mathrm{Z}\left(\mathscr{D}_{\text {pre }}^{2 p}(X, p)\right) \oplus \widetilde{\mathscr{D}}_{\text {pre }}^{2 p-1}(U, p) .
$$

Observe that a weak pre-log-log Green object carries less information than a pre$\log$-log Green object. For instance, the subsequent proposition is not true, in general, for weak Green objects. Nevertheless, weak Green objects appear naturally when considering nonproper intersections, and they are useful as intermediate steps (see [BKK2, Remark 3.55]).

PROPOSITION 1.13

Let $\underline{X}=(X, D)$, where $X$ is a proper smooth real variety and $D$ is a fixed normal crossing divisor. Let $y$ be a p-codimensional cycle on $X$ with support $Y$.

(i) If the class of a cycle $(\omega, g)$ in $H_{\mathscr{D}_{\mathrm{pr}}, Y}^{2 p}(X, p)$ is equal to the class of $y$, then

$$
-2 \partial \bar{\partial}[g]=[\omega]-\delta_{y} .
$$

Here, the current associated to a locally integrable form [.] and the Dirac current associated to a cycle $\delta_{y}$ are defined as in [BKK2, (5.32), Definition 5.35].

(ii) Assume that $y=\sum_{j} n_{j} Y_{j}$ with irreducible subvarieties $Y_{j}$ and certain multiplicities $n_{j}$. If the cycle $(\omega, g)$ represents the class of $y$, then the equality

$$
-\lim _{\varepsilon \rightarrow 0} \int_{\partial B_{\varepsilon}(Y)} \alpha \mathrm{d}^{c} g=\frac{(2 \pi i)^{p-1}}{2} \sum_{j} n_{j} \int_{Y_{j}} \alpha
$$

holds for any differential form $\alpha$; here, $\mathrm{d}^{c}=(1 /(4 \pi i))(\partial-\bar{\partial})$ and $B_{\varepsilon}(Y)$ is an $\varepsilon$-neighborhood of $Y$ such that the orientation of $\partial B_{\varepsilon}(Y)$ is induced from the orientation of $B_{\varepsilon}(Y)$.

Notice that in contrast to the theory by Gillet and Soulé, a pre-log-log Green object is not characterized by (1.3). However, if $\omega$ is a smooth form, then a pre-log-log Green object determines a Green current in the sense of Gillet and Soulé. 
In the sequel, we use (for a cycle $y$, as above) the shorthand

$$
\int_{y} \alpha=\sum_{j} n_{j} \int_{Y_{j}} \alpha,
$$

where, as usual,

$$
\int_{Y_{j}} \alpha=\int_{\widetilde{Y}_{j}} \varphi^{*} \alpha
$$

with $\varphi: \widetilde{Y}_{j} \longrightarrow Y_{j}$ any resolution of singularities of $Y_{j}$.

\subsection{Star products of pre-log-log Green objects}

Let $\underline{X}=(X, D)$ be a proper smooth real variety of dimension $d$ with fixed normal crossing divisor $D$. Moreover, let $Y, Z$ be closed subsets of $X$. Then it is shown in [BKK2] that the product $\bullet$ of Deligne-Beilinson cohomology induces a star product

$$
*: \widehat{H}_{\mathscr{D}_{\mathrm{pre}}, Y}^{n}(X, p) \times \widehat{H}_{\mathscr{D}_{\mathrm{pre}}, Z}^{m}(X, q) \longrightarrow \widehat{H}_{\mathscr{D}_{\mathrm{pre}}, Y \cap Z}^{n+m}(X, p+q),
$$

which is graded commutative, associative, and compatible with the morphisms $\omega$ and cl. We fix a cycle $y \in Z^{p}\left(X_{\mathbb{R}}\right)$ with supp $y \subseteq Y$ and a cycle $z \in Z^{q}\left(X_{\mathbb{R}}\right)$ with $\operatorname{supp} z \subseteq Z$. If $y$ and $z$ intersect properly, there is a well-defined intersection cycle $y \cdot z$. If they do not intersect properly, then the cycle $y \cdot z$ is defined in the Chow group of $X$ with supports on $Y \cap Z$. Let $\mathfrak{g}_{y}=\left(\omega_{y}, \widetilde{g}_{y}\right) \in \widehat{H}_{\mathscr{D}_{\mathrm{pre}}, Y}^{2 p}(X, p)$ and $\mathfrak{g}_{z}=\left(\omega_{z}, \widetilde{g}_{z}\right) \in \widehat{H}_{\mathscr{D}_{\mathrm{pre}}, Z}^{2 q}(X, q)$ be weak pre-log-log Green objects for $y$ and $z$, respectively. Then $\mathfrak{g}_{y} * \mathfrak{g}_{z}$ is a weak pre-log-log Green object for the cycle $y \cdot z$ with support $Y \cap Z$. Moreover, if supp $y=Y$, supp $z=Z$, and $Y$ and $Z$ intersect properly, then $\mathfrak{g}_{y} * \mathfrak{g}_{z}$ is a pre-log-log Green object for the cycle $y \cdot z$. We now recall how to find a representative of this Green object.

Adapting the argument of [Bu, p. 362], we can find an embedded resolution of singularities of $Y \cup Z, \pi: \widetilde{X}_{\mathbb{R}} \rightarrow X_{\mathbb{R}}$, which factors through embedded resolutions of $Y, Z$, and $Y \cap Z$. In particular, we can assume that

$$
\pi^{-1}(Y), \quad \pi^{-1}(Z), \quad \pi^{-1}(Y \cap Z), \quad \pi^{-1}(Y \cap D), \quad \text { and } \quad \pi^{-1}(Z \cap D)
$$

are also normal crossing divisors. Let us denote by $\widehat{Y}$ the normal crossing divisor formed by the components of $\pi^{-1}(Y)$ which are not contained in $\pi^{-1}(Y \cap Z)$. Analogously, we denote by $\widehat{Z}$ the normal crossing divisor formed by the components of $\pi^{-1}(Z)$ which are not contained in $\pi^{-1}(Y \cap Z)$. Then $\widehat{Y}$ and $\widehat{Z}$ are closed subsets of $\tilde{X}$ which do not meet. Therefore there exist two smooth, $F_{\infty}$-invariant functions $\sigma_{Y Z}$ and $\sigma_{Z Y}$ satisfying $0 \leq \sigma_{Y Z}, \sigma_{Z Y} \leq 1, \sigma_{Y Z}+\sigma_{Z Y}=1, \sigma_{Y Z}=1$ in a neighborhood of $\widehat{Y}$, and $\sigma_{Z Y}=1$ in a neighborhood of $\widehat{Z}$. Finally, in the group $\widehat{H}_{\mathscr{D}_{\mathrm{pre}}, Y \cap Z}^{2 p+2 q}(X, p+q)$, we 
then have the identity

$$
\mathfrak{g}_{y} * \mathfrak{g}_{z}=\left(\omega_{y} \wedge \omega_{z},\left(-2 \sigma_{Z Y} g_{y} \wedge \partial \bar{\partial} g_{z}-2 \partial \bar{\partial}\left(\sigma_{Y Z} g_{y}\right) \wedge g_{z}\right)^{\sim}\right) .
$$

In order to compute the arithmetic degree of an arithmetic intersection, we need formulas for the pushforward of certain $*$-products in the top degree of truncated cohomology groups. We make the convention that for $\mathfrak{g}=(\omega, \widetilde{g}) \in \widehat{H}_{\mathscr{D}_{\mathrm{pre}}, \emptyset}^{2 d+2}(X, d)$, we write $\int_{X} \mathfrak{g}$ instead of $\int_{X} g$.

\section{THEOREM 1.14}

Let $\underline{X}$ be as before, and assume that $D=D_{1} \cup D_{2}$, where $D_{1}$ and $D_{2}$ are normal crossing divisors of $X$ satisfying $D_{1} \cap D_{2}=\emptyset$. Let $y$ and $z$ be cycles of $X$ such that supp $y=Y$ and $\operatorname{supp} z \subseteq Z$. Let $\mathfrak{g}_{y}$ be a pre-log-log Green object for $y$, and let $\mathfrak{g}_{z}$ be a weak pre-log-log Green object for $z$ with support $Z$. Assume that $p+q=d+1$, and assume that $Y \cap Z=\emptyset, Y \cap D_{2}=\emptyset$, and $Z \cap D_{1}=\emptyset$. Then

$$
\begin{aligned}
\frac{1}{(2 \pi i)^{d}} \int_{X} \mathfrak{g}_{y} * \mathfrak{g}_{z}=\lim _{\varepsilon \rightarrow 0}( & \frac{1}{(2 \pi i)^{d}} \int_{X \backslash B_{\varepsilon}(D)} g_{y} \wedge \omega_{z} \\
& \left.\quad-\frac{2}{(2 \pi i)^{d-1}} \int_{\partial\left(B_{\varepsilon}\left(D_{1}\right)\right)}\left(g_{z} \wedge \mathrm{d}^{c} g_{y}-g_{y} \wedge \mathrm{d}^{c} g_{z}\right)\right) \\
& +\frac{1}{(2 \pi i)^{q-1}} \int_{\frac{y \backslash\left(y \cap D_{1}\right)}{}} g_{z},
\end{aligned}
$$

where $B_{\varepsilon}\left(D_{j}\right)$ denotes an $\varepsilon$-neighborhood of $D_{j}(j=1,2)$ and $B_{\varepsilon}(D)=B_{\varepsilon}\left(D_{1}\right) \cup$ $B_{\varepsilon}\left(D_{2}\right)$.

\section{Proof}

Let $\widetilde{X}$ be an embedded resolution of $Y \cup Z$ as described above. We write $Y^{\prime}$, respectively, $Z^{\prime}$, for the strict transforms of $Y$, respectively, $Z$; we note that $Y^{\prime} \cap Z^{\prime}=\emptyset$. Furthermore, we write $Y^{\prime \prime}$ for the strict transform of the closure of $Y \backslash(Y \cap D)$. Choosing $\sigma_{Y Z}$ and $\sigma_{Z Y}$ as above, we may assume that $\sigma_{Y Z}$ has value 1 in a neighborhood of $D_{1}$ (since $D_{1} \cap Z=\emptyset$ ) and vanishes in a neighborhood of $D_{2}$.

Using $-2 \partial \bar{\partial}=(4 \pi i) \mathrm{dd}^{c}$, we get, by means of $(1.5)$,

$$
\mathfrak{g}_{y} * \mathfrak{g}_{z}=\left(\omega_{y} \wedge \omega_{z}, 4 \pi i\left(\operatorname{dd}^{c}\left(\sigma_{Y Z} g_{y}\right) \wedge g_{z}+\sigma_{Z Y} g_{y} \wedge \operatorname{dd}^{c} g_{z}\right)^{\sim}\right) .
$$

In order to perform the following calculations, we put

$$
X_{\varepsilon}=\tilde{X} \backslash\left(B_{\varepsilon}(D) \cup B_{\varepsilon}\left(Y^{\prime \prime}\right) \cup B_{\varepsilon}\left(Z^{\prime}\right)\right),
$$


where $B_{\varepsilon}(\cdot)$ denotes an $\varepsilon$-neighborhood of the quantities in question. On $X_{\varepsilon}$, one can split up the integral in question by means of [BKK2, (7.32)]:

$$
\begin{aligned}
& \int_{X_{\varepsilon}}\left(\operatorname{dd}^{c}\left(\sigma_{Y Z} g_{y}\right) \wedge g_{z}+\sigma_{Z Y} g_{y} \wedge \mathrm{dd}^{c} g_{z}\right) \\
& =\int_{X_{\varepsilon}} g_{y} \wedge \mathrm{dd}^{c} g_{z}+\int_{X_{\varepsilon}} \mathrm{d}\left(g_{z} \wedge \mathrm{d}^{c}\left(\sigma_{Y Z} g_{y}\right)-\sigma_{Y Z} g_{y} \wedge \mathrm{d}^{c} g_{z}\right) .
\end{aligned}
$$

Applying Stokes's theorem to the latter integral and using the properties of the function $\sigma_{Y Z}$, we obtain, for sufficiently small $\varepsilon>0$,

$$
\begin{aligned}
\int_{X_{\varepsilon}} \mathrm{d}\left(g_{z} \wedge \mathrm{d}^{c}\left(\sigma_{Y Z} g_{y}\right)-\sigma_{Y Z} g_{y} \wedge \mathrm{d}^{c} g_{z}\right) \\
=-\int_{\partial\left(B_{\varepsilon}\left(D_{1}\right) \cup B_{\varepsilon}\left(Y^{\prime \prime}\right)\right)}\left(g_{z} \wedge \mathrm{d}^{c} g_{y}-g_{y} \wedge \mathrm{d}^{c} g_{z}\right) \\
=-\int_{\partial\left(B_{\varepsilon}\left(D_{1}\right)\right)}\left(g_{z} \wedge \mathrm{d}^{c} g_{y}-g_{y} \wedge \mathrm{d}^{c} g_{z}\right) \\
\quad-\int_{\partial\left(B_{\varepsilon}\left(Y^{\prime \prime}\right) \backslash\left(B_{\varepsilon}\left(Y^{\prime \prime}\right) \cap B_{\varepsilon}\left(D_{1}\right)\right)\right)}\left(g_{z} \wedge \mathrm{d}^{c} g_{y}-g_{y} \wedge \mathrm{d}^{c} g_{z}\right) .
\end{aligned}
$$

Taking into account that $\left(\omega_{y}, \widetilde{g}_{y}\right)$ is a Green object for $y$ and that $g_{z}$ is a smooth $(n-p, n-p)$-form on $B_{\varepsilon}\left(Y^{\prime \prime}\right) \backslash\left(B_{\varepsilon}\left(Y^{\prime \prime}\right) \cap B_{\varepsilon}\left(D_{1}\right)\right)$, we derive, from (1.4),

$$
\begin{aligned}
& -\int_{\partial\left(B_{\varepsilon}\left(Y^{\prime \prime}\right) \backslash\left(B_{\varepsilon}\left(Y^{\prime \prime}\right) \cap B_{\varepsilon}\left(D_{1}\right)\right)\right)}\left(g_{z} \wedge \mathrm{d}^{c} g_{y}-g_{y} \wedge \mathrm{d}^{c} g_{z}\right) \\
& =\frac{(2 \pi i)^{p-1}}{2} \int_{\overline{y \backslash\left(y \cap D_{1}\right)}} g_{z}+f(\varepsilon) .
\end{aligned}
$$

Here, $f(\varepsilon)$ is a continuous function with $\lim _{\varepsilon \rightarrow 0} f(\varepsilon)=0$. Combining (1.6), (1.7), and (1.8), we finally find

$$
\begin{aligned}
\int_{X_{\varepsilon}} \mathfrak{g}_{y} * \mathfrak{g}_{z}=\lim _{\varepsilon \rightarrow 0}( & \int_{X \backslash B_{\varepsilon}(D)} g_{y} \wedge\left(-2 \partial \bar{\partial} g_{z}\right)+(2 \pi i)^{p} \int_{\overline{y \backslash\left(y \cap D_{1}\right)}} g_{z} \\
& \left.-4 \pi i \int_{\partial\left(B_{\varepsilon}\left(D_{1}\right)\right)}\left(g_{z} \wedge \mathrm{d}^{c} g_{y}-g_{y} \wedge \mathrm{d}^{c} g_{z}\right)+f(\varepsilon)\right) .
\end{aligned}
$$

Hence the claim follows.

Observe that if $D$ is empty and $\operatorname{supp} z=Z$, then the formula of Theorem 1.14 specializes to the formula for the star product given by Gillet and Soulé (see [GS1]). Nevertheless, when $D$ is not empty, both terms in the formula of Gillet and Soulé may 
be divergent. Therefore one can view the theory of cohomological arithmetic Chow groups as a device that gives, in the nonsmooth situation, the necessary correction terms.

\subsection{Arithmetic Chow rings with pre-log-log forms}

Let $K$ be a number field, let $A$ be a subring of $K$ with field of fractions $K$, and let $\Sigma$ be a complete set of complex embeddings of $K$ into $\mathbb{C}$. Let $\mathscr{X}$ be an arithmetic variety over $A$ of (relative) dimension $d$ over $S=\operatorname{Spec} A$ (i.e., a regular scheme $\mathscr{X}$ that is flat and quasi-projective over $\operatorname{Spec} A$ ). We let $\mathscr{X}_{\infty}=\bigsqcup_{\sigma \in \Sigma} \mathscr{X}_{\sigma}(\mathbb{C})$. This complex variety has a natural antilinear involution denoted $F_{\infty}$. We denote by $\mathscr{X}_{\mathbb{R}}$ the real variety defined by $\mathscr{X}_{\infty}$ and $F_{\infty}$. Let $D_{K}$ be a fixed normal crossing divisor of $\mathscr{X}_{K}$. We denote by $D$ the induced normal crossing divisor on $\mathscr{X}_{\mathbb{R}}$. In this section, we recall basic properties of the arithmetic Chow groups $\widehat{\mathrm{CH}}^{*}\left(\mathscr{X}, \mathscr{D}_{\text {pre }}\right)$ defined in [BKK2].

Let $\mathrm{Z}^{p}(\mathscr{X})$ be the group formed by cycles on $\mathscr{X}$ of codimension $p$. Given $y \in \mathrm{Z}^{p}(\mathscr{X})$, we write $y_{\infty}=\bigsqcup_{\sigma \in \Sigma} y_{\sigma}(\mathbb{C})$ and let $Y=\operatorname{supp} y_{\infty}$. We define

$$
\widehat{H}_{\mathscr{O}_{\mathrm{pre}}, Y}^{2 p}(\mathscr{X}, p)=\widehat{H}_{\mathscr{D}_{\mathrm{pre}}, Y}^{2 p}\left(\mathscr{X}_{\infty}, p\right)^{F_{\infty}},
$$

and we put

$$
\widehat{H}_{\mathscr{D}_{\mathrm{pre}}, \mathscr{Z} p}^{2 p}(\mathscr{X}, p)=\lim _{\overrightarrow{\mathscr{Z}^{p}}} \widehat{H}_{\mathscr{D}_{\mathrm{pre}}, Y}^{2 p}(\mathscr{X}, p),
$$

where in the limit, $\mathscr{Z}^{p}$ is the set of cycles on $\mathscr{X}_{\infty}$ of codimension at least $p$ ordered by inclusion.

\section{Definition 1.15}

The group of p-codimensional arithmetic cycles on $\mathscr{X}$ is the group

$$
\widehat{\mathrm{Z}}^{p}\left(\mathscr{X}, \mathscr{D}_{\text {pre }}\right)=\left\{\left(y, \mathfrak{g}_{y}\right) \in \mathrm{Z}^{p}(\mathscr{X}) \oplus \widehat{H}_{\mathscr{D}_{\text {pre }}, \mathscr{Z}^{p}}^{2 p}(\mathscr{X}, p) \mid \operatorname{cl}\left(\mathfrak{g}_{y}\right)=\operatorname{cl}\left(y_{\infty}\right)\right\} .
$$

Let $w$ be a codimension $p-1$ irreducible subvariety of $\mathscr{X}$, and let $h \in k(w)^{*}$. Write $h_{\infty}$ for the induced function on $w_{\infty}$, and set $Y=\operatorname{supp}\left(\operatorname{div}\left(h_{\infty}\right)\right)$. Then there is a distinguished pre-log-log Green object $\mathfrak{g}(h) \in \widehat{H}_{\mathscr{P}_{\mathrm{pre}}, Y}^{2 p}(\mathscr{X}, p)$ for $\operatorname{div}\left(h_{\infty}\right)$. We point out that $\mathfrak{g}(h)$ depends only on the class of $h_{\infty}$ in $H_{\mathscr{D}_{\text {pre }}}^{2 p-1}(X \backslash Y, p)$. We write $\widehat{\operatorname{div}}(h)=(\operatorname{div}(h), \mathfrak{g}(h))$ for this arithmetic cycle, and we denote by $\widehat{\operatorname{Rat}}^{p}\left(\mathscr{X}, \mathscr{D}_{\text {pre }}\right)$ the subgroup of $\widehat{Z}^{p}(\mathscr{X}, \mathscr{D}$ pre $)$ generated by arithmetic cycles of the form $\widehat{\operatorname{div}}(h)$. Then the pth arithmetic Chow group of $\mathscr{X}$ with log-log growth along $D$ is defined by

$$
\widehat{\mathrm{CH}}^{p}\left(\mathscr{X}, \mathscr{D}_{\text {pre }}\right)=\widehat{\mathrm{Z}}^{p}\left(\mathscr{X}, \mathscr{D}_{\text {pre }}\right) / \widehat{\mathrm{Rat}}^{p}\left(\mathscr{X}, \mathscr{D}_{\text {pre }}\right) .
$$


A key result of [BKK2] is the definition of an arithmetic intersection product

$$
\widehat{\mathrm{CH}}^{p}\left(\mathscr{X}, \mathscr{D}_{\text {pre }}\right) \otimes \widehat{\mathrm{CH}}^{q}\left(\mathscr{X}, \mathscr{D}_{\text {pre }}\right) \longrightarrow \widehat{\mathrm{CH}}^{p+q}\left(\mathscr{X}, \mathscr{D}_{\text {pre }}\right) \otimes_{\mathbb{Z}} \mathbb{Q},
$$

so that

$$
\widehat{\mathrm{CH}}^{*}\left(\mathscr{X}, \mathscr{D}_{\mathrm{pre}}\right)_{\mathbb{Q}}=\bigoplus_{p \geq 0} \widehat{\mathrm{CH}}^{p}\left(\mathscr{X}, \mathscr{D}_{\mathrm{pre}}\right) \otimes_{\mathbb{Z}} \mathbb{Q}
$$

equipped with this product has the structure of a commutative associative ring. We call $\widehat{C H}^{*}\left(\mathscr{X}, \mathscr{D}_{\text {pre }}\right)_{\mathbb{Q}}$ the arithmetic Chow ring of $\mathscr{X}$ with log-log growth along $D$ (for a detailed description of the arithmetic intersection product, we refer to [BKK2, Theorems $4.18,4.19])$. We now briefly discuss the special case of $p+q=d+1$.

Let $\left(y, \mathfrak{g}_{y}\right) \in \widehat{\mathrm{Z}}^{p}\left(\mathscr{X}, \mathscr{D}_{\text {pre }}\right)$ and $\left(z, \mathfrak{g}_{z}\right) \in \widehat{\mathrm{Z}}^{q}\left(\mathscr{X}, \mathscr{D}_{\text {pre }}\right)$ be such that $y_{\infty}$ and $z_{\infty}$ have proper intersection on $\mathscr{X}$. Since $p+q=d+1$, this means $y_{\infty} \cap z_{\infty}=\emptyset$, and the intersection of $y$ and $z$ defines a class $[y \cdot z]_{\text {fin }}$ in the Chow group with finite support $\mathrm{CH}_{\text {fin }}^{d+1}(\mathscr{X})_{\mathbb{Q}}$. One obtains

$$
\left[\left(y, \mathfrak{g}_{y}\right) \cdot\left(z, \mathfrak{g}_{z}\right)\right]=\left[[y \cdot z]_{\mathrm{fin}}, \mathfrak{g}_{y} * \mathfrak{g}_{z}\right] \in \widehat{\mathrm{CH}}^{d+1}\left(\mathscr{X}, \mathscr{D}_{\mathrm{pre}}\right)_{\mathbb{Q}} .
$$

\section{Definition 1.16}

Let $K$ be a number field, let $\mathcal{O}_{K}$ be its ring of integers, and let $\Sigma$ be a complete set of complex embeddings of $K$ into $\mathbb{C}$. Then $\operatorname{Spec} \mathcal{O}_{K}$ is an arithmetic variety, and due to the product formula for $K$, we have as in [SABK] a well-defined arithmetic degree map

$$
\widehat{\operatorname{deg}}: \widehat{\mathrm{CH}}^{1}\left(\operatorname{Spec} \mathcal{O}_{K}, \mathscr{D}_{\text {pre }}\right) \longrightarrow \mathbb{R}
$$

induced by the assignment

$$
\left(\sum_{\mathfrak{p}_{j} \in S} n_{j} \mathfrak{p}_{j}, \sum_{\sigma \in \Sigma}\left(0, \widetilde{g}_{\sigma}\right)\right) \mapsto \sum_{\mathfrak{p}_{j} \in S} n_{j} \log \left|\mathcal{O}_{K} / \mathfrak{p}_{j}\right|+\sum_{\sigma \in \Sigma} g_{\sigma} .
$$

In particular, this map is a group homomorphism, which is an isomorphism in the case of $K=\mathbb{Q}$; it is common to identify $\widehat{\mathrm{CH}}^{1}\left(\operatorname{Spec}(\mathbb{Z}), \mathscr{D}_{\text {pre }}\right)$ with $\mathbb{R}$.

\section{Remark 1.17}

If $N$ is an integer and $A=\mathcal{O}_{K}[1 / N]$, then we obtain a homomorphism

$$
\widehat{\operatorname{deg}}: \widehat{\mathrm{CH}}^{1}\left(S, \mathscr{D}_{\text {pre }}\right) \longrightarrow \mathbb{R}_{N} \text {, }
$$

where $\mathbb{R}_{N}=\mathbb{R} /\left\langle\sum_{p \mid N} \mathbb{Q} \cdot \log (p)\right\rangle$, and the sum extends over all prime divisors $p$ of $N$. 
If $\mathscr{X}$ is a $d$-dimensional projective arithmetic variety over $A$ and $\pi: \mathscr{X} \rightarrow S$ is the structure morphism, another key result of [BKK2] is the definition of a pushforward morphism

$$
\pi_{*}: \widehat{\mathrm{CH}}^{d+1}\left(\mathscr{X}, \mathscr{D}_{\mathrm{pre}}\right) \longrightarrow \widehat{\mathrm{CH}}^{1}\left(S, \mathscr{D}_{\mathrm{pre}}\right) .
$$

The arithmetic Chow ring $\widehat{\mathrm{CH}}^{*}\left(\mathscr{X}, \mathscr{D}_{\text {pre }}\right)$ is a generalization of the classical construction $\widehat{\mathrm{CH}}^{*}(\mathscr{X})$ due to Gillet and Soulé (see, e.g., [SABK]), in which the differential forms are allowed to have certain log-log singularities along a fixed normal crossing divisor.

THEOREM 1.18

If $\mathscr{X}$ is projective, then there is a commutative diagram

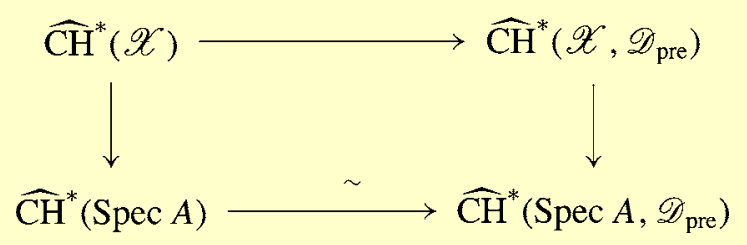

in which the upper morphism is compatible with the product structure, the vertical morphisms are the pushforward morphisms, and the lower morphism is an isomorphism compatible with arithmetic degrees. In particular, this diagram implies the compatibility of the arithmetic intersection numbers that can be computed in both theories.

Remark 1.19

Let $A$ be as in Remark 1.17; then the morphism

$$
\widehat{\operatorname{deg}} \pi_{*}: \widehat{\mathrm{CH}}^{d+1}\left(\mathscr{X}, \mathscr{D}_{\text {pre }}\right) \longrightarrow \mathbb{R}_{N}
$$

is induced by the assignment

$$
\sum_{P \in \mathbb{Z}^{d+1}(\mathscr{X})} n_{P}\left(P, \mathfrak{g}_{P}\right) \mapsto \sum_{P \in \mathbb{Z}^{d+1}(\mathscr{X})} n_{P}\left(\log \#|k(P)|+\frac{1}{(2 \pi i)^{d}} \int_{\mathscr{X}_{\infty}} \mathfrak{g}_{P}\right) .
$$

Here, we used the convention that for $\mathfrak{g}_{P}=\left(\omega_{P}, \widetilde{g}_{P}\right)$, we write $\int_{\mathscr{X}_{\infty}} \mathfrak{g}_{P}$ instead of $\int_{\mathscr{X}_{\infty}} g_{P}$.

In order to ease notation, we sometimes write for $\alpha \in \widehat{\mathrm{CH}}^{d+1}\left(\mathscr{X}, \mathscr{D}_{\text {pre }}\right)$ simply $\alpha$ instead of $\widehat{\operatorname{deg}} \pi_{*}(\alpha)$.

1.5. Pre-log singular hermitian line bundles and Faltings heights

Let $\mathscr{X}$ and $D$ be as in Section 1.4. 


\section{Definition 1.20}

Let $\mathscr{L}$ be a line bundle on $\mathscr{X}$ equipped with an $F_{\infty}$-invariant singular hermitian metric $\|\cdot\|$ on the induced line bundle $\mathscr{L}_{\infty}$ over $\mathscr{X}_{\infty}$. If there is an analytic trivializing cover $\left\{U_{\alpha}, s_{\alpha}\right\}_{\alpha}$ such that, for all $\alpha$,

$$
-\log \left\|s_{\alpha}\right\| \in \Gamma\left(U_{\alpha}, \mathscr{E}_{\mathscr{X}_{\infty}}^{0}\langle\langle D\rangle\rangle_{\mathrm{pre}}\right),
$$

then the metric is called a pre-log singular hermitian metric. The pair $(\mathscr{L},\|\cdot\|)$ is called a pre-log singular hermitian line bundle and denoted by $\overline{\mathscr{L}}$.

LEMMA 1.21

If $\overline{\mathscr{L}}$ is a pre-log singular hermitian line bundle on $\mathscr{X}$, then for any rational section $s$ of $\mathscr{L}$,

$$
(\operatorname{div}(s),(2 \partial \bar{\partial} \log \|s\|,-\log \|s\|)) \in \widehat{Z}^{1}\left(\mathscr{X}, \mathscr{D}_{\text {pre }}\right) .
$$

\section{Proof}

Let $\|\cdot\|_{0}$ be an $F_{\infty}$-invariant smooth hermitian metric on the line bundle $\mathscr{L}_{\infty}$. Since the quotient $\|s\| /\|s\|_{0}$ does not depend on the section $s$, Definition 1.20 implies that

$$
f=-\log \frac{\|s\|}{\|s\|_{0}}
$$

is a pre-log-log function. Consequently, $(2 \partial \bar{\partial} f,-f)$ is a pre-log-log Green object for the empty divisor (see [BKK2, Section 7.7]). Since $\left(2 \partial \bar{\partial} \log \|s\|_{0},-\log \|s\|_{0}\right)$ is a Green object for $\operatorname{div}(s)$ and

$$
(2 \partial \bar{\partial} \log \|s\|,-\log \|s\|)=\left(2 \partial \bar{\partial} \log \|s\|_{0},-\log \|s\|_{0}\right)-\mathrm{a}(f),
$$

we obtain that $(2 \partial \bar{\partial} \log \|s\|,-\log \|s\|)$ is a pre-log-log Green object for $\operatorname{div}(s)$.

It is easy to see that the class of (1.12) depends only on the pair $(\mathscr{L},\|\cdot\|)$. We denote it by $\widehat{\mathrm{c}}_{1}(\overline{\mathscr{L}})$, and we call it the first arithmetic Chern class of $\overline{\mathscr{L}}$.

\section{Definition 1.22}

The arithmetic Picard group $\widehat{\operatorname{Pic}}\left(\mathscr{X}, \mathscr{D}_{\text {pre }}\right)$ is the group of isomorphy classes of pre$\log$ singular hermitian line bundles, where the group structure is given by the tensor product.

We have an inclusion $\widehat{\operatorname{Pic}}(\mathscr{X}) \subseteq \widehat{\operatorname{Pic}}\left(\mathscr{X}, \mathscr{D}_{\text {pre }}\right)$, where $\widehat{\operatorname{Pic}}(\mathscr{X})$ is the arithmetic Picard group defined by Gillet and Soulé. Moreover, the morphism

$$
\widehat{\mathrm{c}}_{1}: \widehat{\operatorname{Pic}}\left(\mathscr{X}, \mathscr{D}_{\mathrm{pre}}\right) \longrightarrow \widehat{\mathrm{CH}}^{1}\left(\mathscr{X}, \mathscr{D}_{\mathrm{pre}}\right) \text {, }
$$


given by equation (1.12), is an isomorphism. Finally, given a pre-log singular hermitian line bundle $\overline{\mathscr{L}}$ on an arithmetic variety of (relative) dimension $d$ over $A$, we write

$$
\overline{\mathscr{L}}^{d+1}=\widehat{\operatorname{deg}} \pi_{*}\left(\widehat{\mathrm{c}}_{1}(\overline{\mathscr{L}})^{d+1}\right),
$$

and we call it the arithmetic self-intersection number of $\overline{\mathscr{L}}$.

Putting $U=\mathscr{X}_{\mathbb{R}} \backslash D$, we write $\mathrm{Z}_{U}^{q}(\mathscr{X})$ for the group of the $q$-codimensional cycles $z$ of $\mathscr{X}$ so that $z_{\mathbb{R}}$ intersects $D$ properly. We introduce $U_{K}=\mathscr{X}_{K} \backslash D_{K}$ and observe that there is a natural injective map $\mathrm{Z}^{q}\left(U_{K}\right) \rightarrow \mathrm{Z}_{U}^{q}(\mathscr{X})$. In [BKK2], a height pairing

$$
(\cdot \mid \cdot): \widehat{\mathrm{CH}}^{p}\left(\mathscr{X}, \mathscr{D}_{\mathrm{pre}}\right) \otimes \mathrm{Z}_{U}^{q}(\mathscr{X}) \longrightarrow \widehat{\mathrm{CH}}^{p+q-d}\left(S, \mathscr{D}_{\text {pre }}\right)_{\mathbb{Q}}
$$

is defined. Observe that since the height of a cycle, whose generic part is supported in $D$, may be infinite, one cannot expect that the height pairing due to Bost, Gillet, and Soulé [BGS] unconditionally generalizes to a height pairing between the arithmetic Chow groups $\widehat{\mathrm{CH}}^{p}\left(\mathscr{X}, \mathscr{D}_{\text {pre }}\right)$ and the whole group of cycles $\mathrm{Z}^{q}(\mathscr{X})$.

We now let $\mathscr{X}, D, U$ be as before, and we let $p, q$ be integers satisfying $p+q=$ $d+1$. Let $z \in \mathrm{Z}_{U}^{q}(\mathscr{X})$ be an irreducible, reduced cycle, and let $\alpha \in \widehat{\mathrm{CH}}^{p}\left(\mathscr{X}, \mathscr{D}_{\text {pre }}\right)$. We represent $\alpha$ by the class of an arithmetic cycle $\left(y, \mathfrak{g}_{y}\right)$, where $y$ is a $p$-codimensional cycle such that $y_{K}$ intersects $z_{K}$ properly and where $\mathfrak{g}_{y}=\left(\omega_{y}, \widetilde{g}_{y}\right)$ is a pre-log-log Green object for $y$. We have

$$
(\alpha \mid z)=\left[\pi_{*}\left([y \cdot z]_{\text {fin }}\right),\left(0, \pi_{\#}\left(\widetilde{g_{y} \wedge} \delta_{z}\right)\right)\right] \in \widehat{\mathrm{CH}}^{1}\left(S, \mathscr{D}_{\text {pre }}\right)_{\mathbb{Q}} .
$$

Here, the quantity $\left.\pi_{\#} \widetilde{\left(g_{y} \wedge\right.} \delta_{z}\right)$ has to be understood as follows. Let $Z=\operatorname{supp} z_{\mathbb{R}}$, and let $\imath: \widetilde{Z} \rightarrow Z$ be a resolution of singularities of $Z$ adapted to $D$. Since $y_{K} \cap z_{K}=\emptyset$, the functoriality of pre-log-log forms shows that $l^{*}\left(g_{y}\right)$ is a pre-log-log form on $\widetilde{Z}$; hence it is locally integrable on $\widetilde{Z}$, and we have

$$
\widetilde{\pi_{\#}\left(g_{y} \wedge \delta_{z}\right)}=\frac{1}{(2 \pi i)^{p-1}} \int_{\widetilde{Z}} l^{*}\left(g_{y}\right) .
$$

The pairing (1.14) is now obtained by linearly extending the above definitions.

If we choose a basic pre-log-log Green form $g_{z}$ for $z$ and put $\mathfrak{g}_{z}=\left(-2 \partial \bar{\partial} g_{z}, \widetilde{g}_{z}\right)$, then the height pairing (1.14) satisfies

$$
(\alpha \mid z)=\pi_{*}\left(\alpha \cdot\left[z, \mathfrak{g}_{z}\right]\right)+\left(0,\left(0, \pi_{\#}\left(\left[\omega\left(\widetilde{\alpha) \wedge g_{z}}\right]_{\mathscr{X}_{\mathbb{R}}}\right)\right) \in \widehat{\mathrm{CH}}^{1}\left(S, \mathscr{D}_{\mathrm{pre}}\right)_{\mathbb{Q}} .\right.\right.
$$

The height pairing (1.14) is of particular interest when $\alpha=\widehat{\mathrm{c}}_{1}(\overline{\mathscr{L}})^{p}$ for some pre-log singular hermitian line bundle $\overline{\mathscr{L}}$ on $\mathscr{X}$. We call the real number

$$
\mathrm{ht}_{\overline{\mathscr{L}}}(z)=\widehat{\operatorname{deg}}\left(\widehat{\mathrm{c}}_{1}(\overline{\mathscr{L}})^{p} \mid z\right)
$$

the Faltings height of $z$ (with respect to $\overline{\mathscr{L}}$ ). 


\section{Remark 1.23}

As in Theorem 1.18, if the hermitian metric of $\overline{\mathscr{L}}$ is smooth, the Faltings height computed in this arithmetic intersection theory agrees with the one defined by Bost, Gillet, and Soulé.

\section{Complex theory of Hilbert modular surfaces}

We begin by recalling some basic facts on Hilbert modular surfaces. This mainly serves to fix notation (for a detailed account, we refer to [Fr3] and [G]).

Let $K$ be a real quadratic field with discriminant $D$. Let $\mathcal{O}_{K}$ be its ring of integers, and let $\mathfrak{d}=(\sqrt{D})$ be the different. We write $x \mapsto x^{\prime}$ for the conjugation in $K$, $\operatorname{tr}(x)=x+x^{\prime}$ for the trace, and $\mathrm{N}(x)=x x^{\prime}$ for the norm of an element. Given an $a \in K$, we write $a \gg 0$ if $a$ is totally positive. Furthermore, we denote by $\varepsilon_{0}>1$ the fundamental unit of $K$. We write $\chi_{D}$ for the quadratic character associated with $K$ given by the Legendre symbol $\chi_{D}(x)=\left(\frac{D}{x}\right)$. The Dirichlet $L$-function corresponding to $\chi_{D}$ is denoted by $L\left(s, \chi_{D}\right)$. Moreover, we write $\zeta(s)$ for the Riemann zeta function.

Let $\mathbb{H}=\{z \in \mathbb{C} ; \Im(z)>0\}$ be the upper complex half-plane. The group $\mathrm{SL}_{2}(\mathbb{R}) \times \mathrm{SL}_{2}(\mathbb{R})$ acts on the product $\mathbb{H}^{2}$ of two copies of $\mathbb{H}$ via Möbius transformations on both factors. As usual, we identify $\mathrm{SL}_{2}(K)$ with a subgroup of $\mathrm{SL}_{2}(\mathbb{R}) \times \mathrm{SL}_{2}(\mathbb{R})$ by the embedding $M \mapsto\left(M, M^{\prime}\right)$, where $M^{\prime}=\left(\begin{array}{c}a^{\prime} \\ c^{\prime} \\ d^{\prime}\end{array}\right)$ denotes the conjugate of the matrix $M=\left(\begin{array}{ll}a & b \\ c & d\end{array}\right)$. If $\mathfrak{a}$ is a fractional ideal of $K$, we write

$$
\Gamma\left(\mathcal{O}_{K} \oplus \mathfrak{a}\right)=\left\{\left(\begin{array}{ll}
a & b \\
c & d
\end{array}\right) \in \mathrm{SL}_{2}(K) ; a, d \in \mathcal{O}_{K}, b \in \mathfrak{a}^{-1}, c \in \mathfrak{a}\right\}
$$

for the Hilbert modular group corresponding to $\mathfrak{a}$. Moreover, we briefly write

$$
\Gamma_{K}=\Gamma\left(\mathcal{O}_{K} \oplus \mathcal{O}_{K}\right)=\mathrm{SL}_{2}\left(\mathcal{O}_{K}\right),
$$

and we denote by $\Gamma_{K}(N)$ the principal congruence subgroup of level $N$, that is, the kernel of the natural homomorphism $\Gamma_{K} \rightarrow \mathrm{SL}_{2}\left(\mathcal{O}_{K} / N \mathcal{O}_{K}\right)$. Throughout, we use $z=\left(z_{1}, z_{2}\right)$ as a standard variable on $\mathbb{H}^{2}$. We denote its real part by $\left(x_{1}, x_{2}\right)$ and its imaginary part by $\left(y_{1}, y_{2}\right)$.

Let $\Gamma \leq \mathrm{SL}_{2}(K)$ be a subgroup that is commensurable with $\Gamma_{K}$. The quotient $\Gamma \backslash \mathbb{H}^{2}$ is called the Hilbert modular surface associated with $\Gamma$. It is a noncompact normal complex space that can be compactified by adding the cusps of $\Gamma$, that is, the $\Gamma$-classes of $\mathbb{P}^{1}(K)$. By the theory of Baily and Borel, the quotient

$$
X(\Gamma)=\Gamma \backslash\left(\mathbb{H}^{2} \cup \mathbb{P}^{1}(K)\right),
$$

together with the Baily-Borel topology, can be given the structure of a normal projective algebraic variety over $\mathbb{C}$. It is called the Baily-Borel compactification of $\Gamma \backslash \mathbb{H}^{2}$. Recall that the cusps of $\Gamma\left(\mathcal{O}_{K} \oplus \mathfrak{a}\right)$ are in bijection with the ideal classes of $K$ by 
mapping $(\alpha: \beta) \in \mathbb{P}^{1}(K)$ to the ideal $\alpha \mathcal{O}_{K}+\beta \mathfrak{a}^{-1}$. So, in particular, the cusp $\infty$ corresponds to the principal class and 0 to the class of $\mathfrak{a}^{-1}$. For any point $\xi \in \mathbb{H}^{2} \cup \mathbb{P}^{1}(K)$, we denote by $\Gamma_{\xi}$ the stabilizer of $\xi$ in $\Gamma$. If $\xi \in \mathbb{H}^{2}$, then the quotient $G=\Gamma_{\xi} /\{ \pm 1\}$ is a finite cyclic group. If $|G|>1$, then $\xi$ is called an elliptic fixed point. Notice that $\Gamma_{K}$ always has elliptic fixed points of orders 2 and 3. On the other hand, $\Gamma_{K}(N)$ acts fixed-point-freely if $N \geq 3$.

If $\mathfrak{a}$ is a fractional ideal from the principal genus of $K$, there are a fractional ideal $\mathfrak{c}$ and a totally positive $\lambda \in K$ such that $\mathfrak{a}=\lambda \mathfrak{c}^{2}$. If $M$ denotes a matrix in

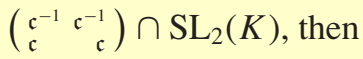

$$
\left(\begin{array}{ll}
\lambda^{-1} & 0 \\
0 & 1
\end{array}\right) M \Gamma_{K} M^{-1}\left(\begin{array}{ll}
\lambda & 0 \\
0 & 1
\end{array}\right)=\Gamma\left(\mathcal{O}_{K} \oplus \mathfrak{a}\right) .
$$

This induces an isomorphism of algebraic varieties over $\mathbb{C}$,

$$
\Gamma_{K} \backslash \mathbb{H}^{2} \longrightarrow \Gamma\left(\mathcal{O}_{K} \oplus \mathfrak{a}\right) \backslash \mathbb{H}^{2}, \quad z \mapsto\left(\begin{array}{cc}
\lambda^{-1} & 0 \\
0 & 1
\end{array}\right) M z .
$$

It extends to an isomorphism $X\left(\Gamma_{K}\right) \rightarrow X\left(\Gamma\left(\mathcal{O}_{K} \oplus \mathfrak{a}\right)\right)$ mapping the cusp $\mathfrak{b}$ of $X\left(\Gamma_{K}\right)$ to the cusp $\mathfrak{b} / \mathfrak{c}$ of $X\left(\Gamma\left(\mathcal{O}_{K} \oplus \mathfrak{a}\right)\right)$. In particular, the cusp $\mathfrak{c}$ is mapped to the cusp $\infty$ of $X\left(\Gamma\left(\mathcal{O}_{K} \oplus \mathfrak{a}\right)\right)$, and $\mathfrak{c}^{-1}$ is mapped to the cusp 0 .

\subsection{Desingularization and the curve lemma}

Throughout, we write $\mathbf{e}(z)=e^{2 \pi i z}$. We denote by $\mathbb{E}(\delta)=\{q \in \mathbb{C} ;|q|<\delta\}$ the $\delta$-disc around the origin, and we put $\mathbb{E}=\mathbb{E}(1)$. Moreover, we write $\dot{\mathbb{E}}=\{q \in \mathbb{E} ; q \neq 0\}$.

The singular locus $X(\Gamma)^{\text {sing }}$ of $X(\Gamma)$ consists of the cusps and the elliptic fixed points. Throughout, we work with desingularizations of $X(\Gamma)$ such that the pullback of the singular locus is a divisor with normal crossings. Given such a desingularization

$$
\pi: \tilde{X}(\Gamma) \longrightarrow X(\Gamma)
$$

we denote this divisor by

$$
\mathbf{D}_{\Gamma}=\pi^{*}\left(X(\Gamma)^{\text {sing }}\right) .
$$

We now present a local description of $\widetilde{X}(\Gamma)$ using the "curve lemma" due to Freitag.

Let $\kappa \in \mathbb{P}^{1}(K)$ be a cusp of $\Gamma$, and let $g \in \mathrm{SL}_{2}(K)$ with $\kappa=g \infty$. By replacing $\Gamma$ by the commensurable group $g^{-1} \Gamma g$, we may assume that $\kappa=\infty$. There are a $\mathbb{Z}$-module $\mathfrak{t} \subset K$ of rank 2 and a finite index subgroup $\Lambda$ of the units of $\mathcal{O}_{K}$ acting on $\mathfrak{t}$ such that $\Gamma_{\infty}$ has finite index in the semidirect product $\mathfrak{t} \rtimes \Lambda$ (see [G, Chapter 2.1]). In particular, if $\gamma \in \Gamma_{\infty}$, then $\gamma\left(z_{1}, z_{2}\right)=\left(\varepsilon z_{1}+\mu, \varepsilon^{\prime} z_{2}+\mu^{\prime}\right)$ for some $\mu \in \mathfrak{t}$ and some totally positive unit $\varepsilon \in \Lambda$. A fundamental system of open neighborhoods 
of $\infty \in X(\Gamma)$ is given by

$$
V_{C}=\Gamma_{\infty} \backslash\left\{\left(z_{1}, z_{2}\right) \in \mathbb{H}^{2} ; y_{1} y_{2}>C\right\} \cup \infty, \quad C>0 .
$$

Let $C>0$. Let $a \in \tilde{X}(\Gamma)$ be a point with $\pi(a)=\infty$, and let $U \subset \widetilde{X}(\Gamma)$ be a small open neighborhood of $a$ such that $\pi(U) \subset V_{C}$. Possibly replacing $U$ by a smaller neighborhood, after a biholomorphic change of coordinates we may assume that $U=\mathbb{E}^{2}$ is the product of two unit discs, $a=(0,0)$, and that $\pi^{*} \infty=\operatorname{div}\left(q_{1}^{\alpha} q_{2}^{\beta}\right)$ on $U$ with nonnegative integers $\alpha, \beta$.

The desingularization map induces a holomorphic map $\mathbb{E}^{2} \rightarrow V_{C}$, which we also denote by $\pi$. If we restrict it to $\dot{\mathbb{E}}^{2}$, we get a holomorphic map $\dot{\mathbb{E}}^{2} \rightarrow \Gamma_{\infty} \backslash \mathbb{H}^{2}$. Lifting it to the universal covers, we obtain a commutative diagram

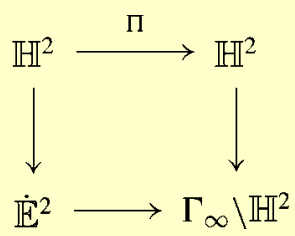

Here, $\Pi$ is a holomorphic function satisfying

$$
\begin{aligned}
& \Pi\left(\tau_{1}+1, \tau_{2}\right)=\left(\begin{array}{cc}
\varepsilon_{1} & 0 \\
0 & \varepsilon_{1}^{\prime}
\end{array}\right) \Pi\left(\tau_{1}, \tau_{2}\right)+\left(\begin{array}{l}
\mu_{1} \\
\mu_{1}^{\prime}
\end{array}\right), \\
& \Pi\left(\tau_{1}, \tau_{2}+1\right)=\left(\begin{array}{cc}
\varepsilon_{2} & 0 \\
0 & \varepsilon_{2}^{\prime}
\end{array}\right) \Pi\left(\tau_{1}, \tau_{2}\right)+\left(\begin{array}{l}
\mu_{2} \\
\mu_{2}^{\prime}
\end{array}\right),
\end{aligned}
$$

with $\mu_{1}, \mu_{2} \in \mathfrak{t}$ and totally positive units $\varepsilon_{1}, \varepsilon_{2} \in \Lambda$.

\section{LEMMA 2.1}

Let $\Pi: \mathbb{H}^{2} \rightarrow \mathbb{H}^{2}$ be a holomorphic function satisfying (2.10) and (2.11). Then $\varepsilon_{1}=\varepsilon_{2}=1$, and there is a holomorphic function $H: \mathbb{E}^{2} \rightarrow \mathbb{H}^{2}$ such that

$$
\Pi\left(\tau_{1}, \tau_{2}\right)=\left(\begin{array}{l}
\Pi_{1}\left(\tau_{1}, \tau_{2}\right) \\
\Pi_{2}\left(\tau_{1}, \tau_{2}\right)
\end{array}\right)=\left(\begin{array}{c}
\mu_{1} \tau_{1}+\mu_{2} \tau_{2} \\
\mu_{1}^{\prime} \tau_{1}+\mu_{2}^{\prime} \tau_{2}
\end{array}\right)+H\left(q_{1}, q_{2}\right),
$$

where $q_{1}=\mathbf{e}\left(\tau_{1}\right)$ and $q_{2}=\mathbf{e}\left(\tau_{2}\right)$. Moreover, $\mu_{1}, \mu_{2}$, and their conjugates are nonnegative.

\section{Proof}

Applying the "curve lemma" (see [Fr1, Satz 1, Hilfssatz 2]) to the functions $\mathbb{H} \rightarrow \mathbb{H}$ given by $\tau_{i} \mapsto \Pi_{j}\left(\tau_{1}, \tau_{2}\right)$ with $i, j \in\{1,2\}$, one finds that $\Pi\left(\tau_{1}, \tau_{2}\right)$ has the form (2.12) with $\mu_{1}, \mu_{2} \in \mathfrak{t}$. The fact that $\Im\left(\Pi_{j}\left(\tau_{1}, \tau_{2}\right)\right)$ is positive implies that $\mu_{1}, \mu_{2}$, and their conjugates have to be nonnegative. 


\section{Remark 2.2}

The properties of the Baily-Borel topology on $X(\Gamma)$ imply that the exceptional divisor $\pi^{*}(\infty)$ contains the component $\left\{q_{j}=0\right\}$ if and only if $\mu_{j}$ is totally positive.

Now, let $\xi \in \mathbb{H}^{2}$ be an elliptic fixed point of $\Gamma$, let $G$ be the cyclic group $\Gamma_{\xi} /\{ \pm 1\}$, and let $n=|G|$. Let $V \subset \mathbb{H}^{2}$ be a small open neighborhood of $\xi$ on which $\Gamma_{\xi}$ acts. Then $\Gamma_{\xi} \backslash V$ is an open neighborhood of $\xi \in \Gamma \backslash \mathbb{H}^{2}$. Let $a \in \tilde{X}(\Gamma)$ be a point with $\pi(a)=\xi$, and let $U \subset \tilde{X}(\Gamma)$ be an open neighborhood of $a$ such that $\pi(U) \subset \Gamma_{\xi} \backslash V$. Without loss of generality, we may assume that $U=\mathbb{E}^{2}$ is the product of two unit $\operatorname{discs}, a=(0,0)$, and that $\pi^{*} \xi=\operatorname{div}\left(q_{1}^{\alpha} q_{2}^{\beta}\right)$ on $U$.

The desingularization map induces a holomorphic map $\mathbb{E}^{2} \rightarrow \Gamma_{\xi} \backslash V$. Arguing as in $[$ Fr2, Hilfssatz 5.19, p. 200] and using the fact that $|G|=n$, we get a commutative diagram of holomorphic maps

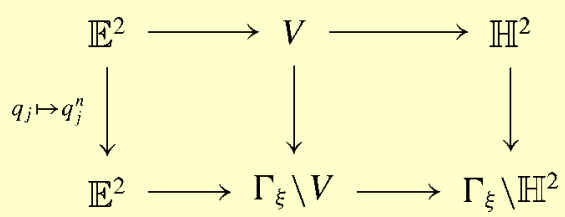

From this, one derives an analogue of the curve lemma for the elliptic fixed points.

There is a (unique up-to-a-positive multiple) symmetric $\left(\mathrm{SL}_{2}(\mathbb{R}) \times \mathrm{SL}_{2}(\mathbb{R})\right.$ )invariant Kähler metric on $\mathbb{H}^{2}$. Its corresponding $(1,1)$-form is given by

$$
\omega=\frac{1}{4 \pi}\left(\frac{\mathrm{d} x_{1} \mathrm{~d} y_{1}}{y_{1}^{2}}+\frac{\mathrm{d} x_{2} \mathrm{~d} y_{2}}{y_{2}^{2}}\right) .
$$

PROPOSITION 2.3

The form $\omega$ induces a pre-log-log form on $\widetilde{X}(\Gamma)$ with respect to $\mathbf{D}_{\Gamma}$.

\section{Proof}

We show that if $\kappa$ is a cusp of $\Gamma$, and $a \in \widetilde{X}(\Gamma)$ with $\pi(a)=\kappa$, then $\pi^{*} \omega$ satisfies the growth conditions of Definition 1.2 in a small neighborhood of $a$. The corresponding assertion for the elliptic fixed points is easy and is left to the reader.

Without loss of generality, we may assume that $\kappa=\infty$ and that $\pi$ looks locally near $a$ as in (2.9).

By means of the $\Gamma_{\infty}$-invariant function $\log \left(y_{1} y_{2}\right)$, we may write $\omega=$ $(1 /(2 \pi i)) \partial \bar{\partial} \log \left(y_{1} y_{2}\right)$. Using the notation of Lemma 2.1, we see that

$$
\Pi^{*}\left(\begin{array}{l}
y_{1} \\
y_{2}
\end{array}\right)=-\frac{1}{2 \pi}\left(\begin{array}{l}
\mu_{1} \log \left|q_{1}\right|+\mu_{2} \log \left|q_{2}\right| \\
\mu_{1}^{\prime} \log \left|q_{1}\right|+\mu_{2}^{\prime} \log \left|q_{2}\right|
\end{array}\right)+\Im\left(\begin{array}{l}
H_{1}\left(q_{1}, q_{2}\right) \\
H_{2}\left(q_{1}, q_{2}\right)
\end{array}\right) .
$$


Consequently,

$$
\pi^{*}\left(\log \left(y_{1} y_{2}\right)\right)=\log \left(-\frac{1}{4 \pi} g_{1}\left(q_{1}, q_{2}\right)\right)+\log \left(-\frac{1}{4 \pi} g_{2}\left(q_{1}, q_{2}\right)\right),
$$

where

$$
\begin{aligned}
& g_{1}\left(q_{1}, q_{2}\right)=\mu_{1} \log \left|q_{1}\right|^{2}+\mu_{2} \log \left|q_{2}\right|^{2}-4 \pi \Im H_{1}\left(q_{1}, q_{2}\right), \\
& g_{2}\left(q_{1}, q_{2}\right)=\mu_{1}^{\prime} \log \left|q_{1}\right|^{2}+\mu_{2}^{\prime} \log \left|q_{2}\right|^{2}-4 \pi \Im H_{2}\left(q_{1}, q_{2}\right) .
\end{aligned}
$$

Hence we find

$$
\begin{aligned}
2 \pi i \pi^{*}(\omega)= & \partial \bar{\partial} \pi^{*}\left(\log \left(y_{1} y_{2}\right)\right) \\
= & -\frac{1}{g_{1}\left(q_{1}, q_{2}\right)^{2}}\left(\mu_{1} \frac{\mathrm{d} q_{1}}{q_{1}}+\mu_{2} \frac{\mathrm{d} q_{2}}{q_{2}}-4 \pi \partial \Im H_{1}\right) \\
& \times\left(\mu_{1} \frac{\mathrm{d} \bar{q}_{1}}{\bar{q}_{1}}+\mu_{2} \frac{\mathrm{d} \bar{q}_{2}}{\bar{q}_{2}}-4 \pi \bar{\partial} \Im H_{1}\right) \\
& -\frac{1}{g_{2}\left(q_{1}, q_{2}\right)^{2}}\left(\mu_{1}^{\prime} \frac{\mathrm{d} q_{1}}{q_{1}}+\mu_{2}^{\prime} \frac{\mathrm{d} q_{2}}{q_{2}}-4 \pi \partial \Im H_{2}\right) \\
& \times\left(\mu_{1}^{\prime} \frac{\mathrm{d} \bar{q}_{1}}{\bar{q}_{1}}+\mu_{2}^{\prime} \frac{\mathrm{d} \bar{q}_{2}}{\bar{q}_{2}}-4 \pi \bar{\partial} \Im H_{2}\right) \\
& -4 \pi \frac{\partial \bar{\partial} \Im H_{1}}{g_{1}\left(q_{1}, q_{2}\right)}-4 \pi \frac{\partial \bar{\partial} \Im H_{2}}{g_{2}\left(q_{1}, q_{2}\right)} .
\end{aligned}
$$

Since $H$ is holomorphic, this differential form has $\log$-log growth along $\pi^{*}(\infty)$.

It follows that the volume form

$$
\omega^{2}=\frac{1}{8 \pi^{2}} \frac{\mathrm{d} x_{1} \mathrm{~d} y_{1}}{y_{1}^{2}} \frac{\mathrm{d} x_{2} \mathrm{~d} y_{2}}{y_{2}^{2}}
$$

is also a pre-log-log form. It is well known that

$$
\int_{\widetilde{X}_{\left(\Gamma_{K}\right)}} \omega^{2}=\frac{1}{8 \pi^{2}} \int_{\Gamma_{K} \backslash \mathbb{H}^{2}} \frac{\mathrm{d} x_{1} \mathrm{~d} y_{1}}{y_{1}^{2}} \frac{\mathrm{d} x_{2} \mathrm{~d} y_{2}}{y_{2}^{2}}=\zeta_{K}(-1)
$$

(see, e.g., [G, p. 59]). Here, $\zeta_{K}(s)$ is the Dedekind zeta function of $K$.

\subsection{Hilbert modular forms and the Petersson metric}

Let $k$ be an integer, and let $\chi$ be a character of $\Gamma$. A meromorphic function $F$ on $\mathbb{H}^{2}$ is called a Hilbert modular form of weight $k$ (with respect to $\Gamma$ and $\chi$ ) if it satisfies

$$
F\left(\gamma z_{1}, \gamma^{\prime} z_{2}\right)=\chi(\gamma)\left(c z_{1}+d\right)^{k}\left(c^{\prime} z_{2}+d^{\prime}\right)^{k} F\left(z_{1}, z_{2}\right)
$$


for all $\gamma=\left(\begin{array}{ll}a & b \\ c & d\end{array}\right) \in \Gamma$. If $F$ is holomorphic on $\mathbb{H}^{2}$, it is called a holomorphic Hilbert modular form. Then, by the Koecher principle, $F$ is automatically holomorphic at the cusps. We denote the vector space of holomorphic Hilbert modular forms of weight $k$ (with respect to $\Gamma$ and trivial character) by $M_{k}(\Gamma)$. A holomorphic Hilbert modular form $F$ has a Fourier expansion at the cusp $\infty$ of the form

$$
F\left(z_{1}, z_{2}\right)=a_{0}+\sum_{\substack{\nu \in \mathfrak{t} \\ \nu \gg 0}} a_{v} \mathbf{e}\left(v z_{1}+v^{\prime} z_{2}\right),
$$

and it has analogous expansions at the other cusps. The sum runs through all totally positive $v$ in a suitable $\mathbb{Z}$-module $\mathfrak{t} \subset K$ of rank 2. For instance, if $\Gamma=\Gamma\left(\mathcal{O}_{K} \oplus \mathfrak{a}\right)$, then $\mathfrak{t}$ is equal to $\mathfrak{a} \mathfrak{d}^{-1}$. Any Hilbert modular form is the quotient of two holomorphic forms. We say that a Hilbert modular form has rational Fourier coefficients, if it is the quotient of two holomorphic Hilbert modular forms with rational Fourier coefficients.

Meromorphic (holomorphic) modular forms of weight $k$ can be interpreted as rational (global) sections of the sheaf $\mathscr{M}_{k}(\Gamma)$ of modular forms. If we write $p: \mathbb{H}^{2} \rightarrow$ $\Gamma \backslash \mathbb{H}^{2}$ for the canonical projection, then the sections over an open subset $U \subset \Gamma \backslash \mathbb{H}^{2}$ are holomorphic functions on $p^{-1}(U)$, which satisfy the transformation law (2.20). This defines a coherent analytic sheaf on $\Gamma \backslash \mathbb{H}^{2}$, which is actually algebraic. By the Koecher principle, it extends to an algebraic sheaf on $X(\Gamma)$. By the theory of Baily and Borel, there is a positive integer $n_{\Gamma}$ such that $\mathscr{M}_{k}(\Gamma)$ is a line bundle if $n_{\Gamma} \mid k$, and

$$
X(\Gamma) \cong \operatorname{Proj}\left(\bigoplus_{k \geq 0, n_{\Gamma} \mid k} M_{k}(\Gamma)\right)
$$

(see [G, p. 44], [C, p. 549]). The line bundle of modular forms of weight $k$ (divisible by $n_{\Gamma}$ ) on $\tilde{X}(\Gamma)$ is defined as the pullback $\pi^{*} \mathscr{M}_{k}(\Gamma)$. By abuse of notation, we also denote it by $\mathscr{M}_{k}(\Gamma)$. In the same way, if $F$ is a Hilbert modular form of weight $k$, we simply write $F$ for the section $\pi^{*}(F)$ on $\widetilde{X}$. The $\operatorname{divisor} \operatorname{div}(F)$ of $F$ decomposes into

$$
\operatorname{div}(F)=\operatorname{div}(F)^{\prime}+\sum_{j} n_{j} E_{j} .
$$

Here, $\operatorname{div}(F)^{\prime}$ denotes the strict transform of the divisor of the modular form $F$ on $X(\Gamma)$, and $E_{j}$ are the irreducible components of the exceptional divisor $\mathbf{D}_{\Gamma}$. The multiplicities $n_{j}$ are determined by the orthogonality relations

$$
\operatorname{div}(F) \cdot E_{j}=0 .
$$

\section{Definition 2.4}

If $F \in \mathscr{M}_{k}(\Gamma)(U)$ is a rational section over an open subset $U \subset \Gamma \backslash \mathbb{H}^{2}$, we define its 
Petersson metric by

$$
\left\|F\left(z_{1}, z_{2}\right)\right\|_{\mathrm{Pet}}^{2}=\left|F\left(z_{1}, z_{2}\right)\right|^{2}\left(16 \pi^{2} y_{1} y_{2}\right)^{k} .
$$

This defines a hermitian metric on the line bundle of modular forms of weight $k$ on $\Gamma \backslash \mathbb{H}^{2}$. We now study how it extends to $\widetilde{X}(\Gamma)$.

\section{PROPOSITION 2.5}

The Petersson metric on the line bundle $\mathscr{M}_{k}(\Gamma)$ of modular forms on $\widetilde{X}(\Gamma)$ is a pre-log singular hermitian metric (with respect to $\mathbf{D}_{\Gamma}$ ).

\section{Proof}

We have to verify the conditions of Definition 1.20 locally for the points of $\mathbf{D}_{\Gamma}$. Here, we consider only the points above the cusps of $X(\Gamma)$. The corresponding assertion for the elliptic fixed points (if there are any) is left to the reader.

Let $\kappa$ be a cusp, and let $a \in \widetilde{X}(\Gamma)$ with $\pi(a)=\kappa$. Moreover, let $F$ be a trivializing section of $\mathscr{M}_{k}(\Gamma)$ over a small neighborhood of $a$. We have to show that $\log \|F\|_{\text {Pet }}$ satisfies the growth conditions of Definition 1.3. Without loss of generality, we may assume that $\kappa=\infty$ and that $\pi$ looks locally near $a$ as in (2.9). It suffices to show that $\pi^{*} \log \left(y_{1} y_{2}\right)$ is a pre-log-log form near $a$.

That $\pi^{*} \log \left(y_{1} y_{2}\right)$ and $\partial \bar{\partial} \pi^{*} \log \left(y_{1} y_{2}\right)$ have $\log$-log growth along $\mathbf{D}_{\Gamma}$ follows from (2.16) and (2.17) in the proof of Lemma 2.3. Using the notation of Lemma 2.3, we see that

$$
\begin{aligned}
\bar{\partial} \pi^{*} \log \left(y_{1} y_{2}\right)= & \frac{1}{g_{1}\left(q_{1}, q_{2}\right)}\left(\mu_{1} \frac{\mathrm{d} \bar{q}_{1}}{\bar{q}_{1}}+\mu_{2} \frac{\mathrm{d} \bar{q}_{2}}{\bar{q}_{2}}-4 \pi \bar{\partial} \Im H_{1}\right) \\
& +\frac{1}{g_{2}\left(q_{1}, q_{2}\right)}\left(\mu_{1}^{\prime} \frac{\mathrm{d} \bar{q}_{1}}{\bar{q}_{1}}+\mu_{2}^{\prime} \frac{\mathrm{d} \bar{q}_{2}}{\bar{q}_{2}}-4 \pi \bar{\partial} \Im H_{2}\right) .
\end{aligned}
$$

Since $H$ is holomorphic, we may infer that $\bar{\partial} \pi^{*} \log \left(y_{1} y_{2}\right)$ has $\log$-log growth along $\mathbf{D}_{\Gamma}$. Analogously, we see that $\partial \pi^{*} \log \left(y_{1} y_{2}\right)$ has $\log$-log growth.

The first Chern form $\mathrm{c}_{1}\left(\mathscr{M}_{k}(\Gamma),\|\cdot\|_{\text {Pet }}\right)$ of the line bundle $\mathscr{M}_{k}(\Gamma)$ equipped with the Petersson metric is given by

$$
\mathrm{c}_{1}\left(\mathscr{M}_{k}(\Gamma),\|\cdot\|_{\mathrm{Pet}}\right)=2 \pi i k \cdot \omega .
$$

Definition 2.6

If $F$ is a Hilbert modular form for $\Gamma$, then we denote the Green object for $\operatorname{div}(F)$ by

$$
\mathfrak{g}(F)=\left(2 \partial \bar{\partial} \log \|F\|_{\text {Pet }},-\log \|F\|_{\text {Pet }}\right) .
$$




\section{Remark 2.7}

In view of (2.19), the geometric self-intersection number $\mathscr{M}_{k}(\Gamma)^{2}$ of the line bundle of modular forms of weight $k$ is equal to $k^{2}\left[\Gamma_{K}: \Gamma\right] \zeta_{K}(-1)$.

\subsection{Green functions for Hirzebruch-Zagier divisors}

From now on, we assume that the discriminant $D$ of the real quadratic field $K$ is a prime. This implies that the fundamental unit $\varepsilon_{0}$ has norm -1 .

We consider the rational quadratic space $V$ of signature $(2,2)$ of matrices $A=$ $\left(\begin{array}{ll}a & v \\ v^{\prime} & b\end{array}\right)$ with $a, b \in \mathbb{Q}$ and $v \in K$, with the quadratic form $q(A)=\operatorname{det}(A)$. For a fractional ideal $\mathfrak{a}$ of $K$, we consider the lattices

$$
\begin{aligned}
L(\mathfrak{a}) & =\left\{\left(\begin{array}{cc}
a & v \\
v^{\prime} & b
\end{array}\right) ; a \in \mathrm{N}(\mathfrak{a}) \mathbb{Z}, b \in \mathbb{Z}, \text { and } v \in \mathfrak{a}\right\}, \\
L^{\prime}(\mathfrak{a}) & =\left\{\left(\begin{array}{cc}
a & v \\
v^{\prime} & b
\end{array}\right) ; a \in \mathrm{N}(\mathfrak{a}) \mathbb{Z}, b \in \mathbb{Z}, \text { and } v \in \mathfrak{a} \mathfrak{d}^{-1}\right\} .
\end{aligned}
$$

Notice that the dual of $L(\mathfrak{a})$ is $(1 / \mathrm{N}(\mathfrak{a})) L^{\prime}(\mathfrak{a})$. The group $\mathrm{SL}_{2}(K)$ acts on $V$ by $\gamma \cdot A=$ $\gamma A \gamma^{\prime t}$ for $\gamma \in \mathrm{SL}_{2}(K)$. Under this action, $\Gamma\left(\mathcal{O}_{K} \oplus \mathfrak{a}^{-1}\right)$ preserves the lattices $L(\mathfrak{a})$ and $L^{\prime}(\mathfrak{a})$. In particular, one obtains an injective homomorphism $\Gamma\left(\mathcal{O}_{K} \oplus \mathfrak{a}^{-1}\right) /\{ \pm 1\} \rightarrow$ $\mathrm{O}(L(\mathfrak{a}))$ into the orthogonal group of $L(\mathfrak{a})$.

Let $m$ be a positive integer. Recall that the subset

$$
\bigcup_{\substack{A=\left(\begin{array}{cc}
a & v \\
v^{\prime} & b
\end{array}\right) \in L^{\prime}(\mathfrak{a}) \\
\operatorname{det}(A)=m \mathrm{~N}(\mathfrak{a}) / D}}\left\{\left(z_{1}, z_{2}\right) \in \mathbb{H}^{2} ; a z_{1} z_{2}+v z_{1}+v^{\prime} z_{2}+b=0\right\}
$$

defines a $\Gamma\left(\mathcal{O}_{K} \oplus \mathfrak{a}\right)$-invariant divisor $T_{\mathfrak{a}}(m)$ on $\mathbb{H}^{2}$, the Hirzebruch-Zagier divisor of discriminant $m$. It is the inverse image of an algebraic divisor on the quotient $\Gamma\left(\mathcal{O}_{K} \oplus\right.$ $\mathfrak{a}) \backslash \mathbb{H}^{2}$, which is also denoted by $T_{\mathfrak{a}}(m)$. Here, we understand that all irreducible components of $T_{\mathfrak{a}}(m)$ are assigned the multiplicity 1. (There is no ramification in codimension 1.) The divisor $T_{\mathfrak{a}}(m)$ is nonzero if and only if $\chi_{D}(m) \neq-1$. If $m$ is square free, then since $D$ is prime, $T_{\mathfrak{a}}(m)$ is irreducible (see [HZ], [G, Chapter 5]). Moreover, $T_{\mathfrak{a}}(m)$ and $T_{\mathfrak{a}}(n)$ intersect properly if and only if $m n$ is not a square.

Since there is only one genus, there are a fractional ideal $\mathfrak{c}$ and a totally positive

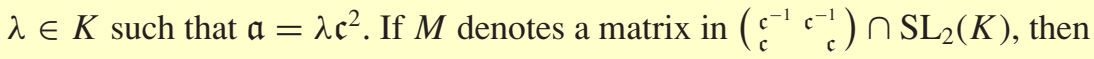

$$
\left(\left(\begin{array}{cc}
\lambda^{-1} & 0 \\
0 & 1
\end{array}\right) M\right)^{-t} \cdot L\left(\mathcal{O}_{K}\right)=\frac{1}{\mathrm{~N}(\mathfrak{c})} L(\mathfrak{a}), \quad\left(\left(\begin{array}{cc}
\lambda^{-1} & 0 \\
0 & 1
\end{array}\right) M\right)^{-t} \cdot L^{\prime}\left(\mathcal{O}_{K}\right)=\frac{1}{\mathrm{~N}(\mathfrak{c})} L^{\prime}(\mathfrak{a}) .
$$

This implies that the isomorphism (2.5) takes $T_{\mathcal{O}_{K}}(m)$ to $T_{\mathfrak{a}}(m)$.

We are mainly interested in the case of $\mathfrak{a}=\mathcal{O}_{K}$. To lighten the notation, we briefly write $L=L\left(\mathcal{O}_{K}\right), L^{\prime}=L^{\prime}\left(\mathcal{O}_{K}\right)$, and $T(m)=T_{\mathcal{O}_{K}}(m)$. 


\section{Definition 2.8}

Let $m$ be a positive integer with $\chi_{D}(m) \neq-1$. If $m$ is the norm of an ideal in $\mathcal{O}_{K}$, then $T(m)$ is a noncompact divisor on $\Gamma_{K} \backslash \mathbb{H}^{2}$, birational to a linear combination of modular curves. In this case, we say that $T(m)$ is isotropic. If $m$ is not the norm of an ideal in $\mathcal{O}_{K}$, then $T(m)$ is a compact divisor on $\Gamma_{K} \backslash \mathbb{H}^{2}$, birational to a linear combination of Shimura curves. In that case, we say that $T(m)$ is anisotropic.

These notions are compatible with the description of $\Gamma_{K} \backslash \mathbb{H}^{2}$ and the divisors $T(m)$ as arithmetic quotients corresponding to orthogonal groups of type $\mathrm{O}(2,2)$ and $\mathrm{O}(2,1)$, respectively (see Section 4.2). Here, isotropic (anisotropic) Hirzebruch-Zagier divisors are given by isotropic (anisotropic) rational quadratic spaces of signature $(2,1)$.

In [Br1, Section 3], a certain Green function $\Phi_{m}\left(z_{1}, z_{2}, s\right)$ was constructed which is associated to the divisor $T(m)$. We briefly recall some of its properties. For $s \in \mathbb{C}$ with $\Re(s)>1$, the function $\Phi_{m}\left(z_{1}, z_{2}, s\right)$ is defined by

$$
\Phi_{m}\left(z_{1}, z_{2}, s\right)=\sum_{\substack{a, b \in \mathbb{Z} \\ \lambda \in \mathcal{D}^{-1} \\ a b-\mathrm{N}(\lambda)=m / D}} Q_{s-1}\left(1+\frac{\left|a z_{1} z_{2}+\lambda z_{1}+\lambda^{\prime} z_{2}+b\right|^{2}}{2 y_{1} y_{2} m / D}\right) .
$$

Here, $Q_{s-1}(t)$ is the Legendre function of the second kind (see [AS, Section 8]), defined by

$$
Q_{s-1}(t)=\int_{0}^{\infty}\left(t+\sqrt{t^{2}-1} \cosh u\right)^{-s} \mathrm{~d} u \quad(t>1, \Re(s)>0) .
$$

The sum in (2.27) converges normally for $\Re(s)>1$ and $\left(z_{1}, z_{2}\right) \in \mathbb{H}^{2}-T(m)$. This implies that $\Phi_{m}\left(z_{1}, z_{2}, s\right)$ is invariant under $\Gamma_{K}$. It has a Fourier expansion

$$
\Phi_{m}\left(z_{1}, z_{2}, s\right)=u_{0}\left(y_{1}, y_{2}, s\right)+\sum_{\substack{v \in \mathfrak{J}^{-1} \\ v \neq 0}} u_{v}\left(y_{1}, y_{2}, s\right) \mathbf{e}\left(v x_{1}+v^{\prime} x_{2}\right),
$$

which converges for $y_{1} y_{2}>m / D$ and $\left(z_{1}, z_{2}\right) \notin T(m)$. As a function in $s$, the latter sum over $v \neq 0$ converges normally for $\Re(s)>3 / 4$. The constant term is a meromorphic function in $s$ with a simple pole at $s=1$. A refinement of these facts can be used to show that $\Phi_{m}\left(z_{1}, z_{2}, s\right)$ has a meromorphic continuation in $s$ to $\{s \in \mathbb{C} ; \Re(s)>3 / 4\}$. Up to a simple pole in $s=1$, it is holomorphic in this domain (see [Br1, Theorem 1]).

We denote by

$$
\Delta_{1}=y_{1}^{2}\left(\frac{\partial^{2}}{\partial x_{1}^{2}}+\frac{\partial^{2}}{\partial y_{1}^{2}}\right), \quad \Delta_{2}=y_{2}^{2}\left(\frac{\partial^{2}}{\partial x_{2}^{2}}+\frac{\partial^{2}}{\partial y_{2}^{2}}\right)
$$


the $\left(\mathrm{SL}_{2}(\mathbb{R}) \times \mathrm{SL}_{2}(\mathbb{R})\right)$-invariant Laplace operators on $\mathbb{H}^{2}$. The differential equation for $Q_{s-1}(t)$ implies that

$$
\Delta_{j} \Phi_{m}\left(z_{1}, z_{2}, s\right)=s(s-1) \Phi_{m}\left(z_{1}, z_{2}, s\right) .
$$

Because $Q_{s-1}(t)=-(1 / 2) \log (t-1)+O(1)$ for $t \rightarrow 1$, the function $\Phi_{m}\left(z_{1}, z_{2}, s\right)$ has a logarithmic singularity along $T(m)$ of type $-\log |f|^{2}$, where $f$ is a local equation.

The Fourier expansion (2.29) of $\Phi_{m}\left(z_{1}, z_{2}, s\right)$ was determined in [Br1]. It follows from [Br1, identity (19), Lemmas 1,2$]$ that the constant term is given by

$$
\begin{aligned}
u_{0}\left(y_{1}, y_{2}, s\right)= & \frac{2 \pi}{(2 s-1)} \sum_{\substack{\lambda \in \mathcal{D}^{-1} \\
\mathrm{~N}(\lambda)=-m / D}} \max \left(\left|\lambda y_{1}\right|,\left|\lambda^{\prime} y_{2}\right|\right)^{1-s} \min \left(\left|\lambda y_{1}\right|,\left|\lambda^{\prime} y_{2}\right|\right)^{s} \\
& +\frac{\pi \Gamma(s-1 / 2)^{2}}{\sqrt{D} \Gamma(2 s)}\left(\frac{4 m}{D}\right)^{s}\left(y_{1} y_{2}\right)^{1-s} \sum_{a=1}^{\infty} G_{a}(m, 0) a^{-2 s}
\end{aligned}
$$

Here, $G_{a}(m, v)$ is the finite exponential sum

$$
G_{a}(m, v)=\sum_{\substack{\lambda \in \mathcal{O}^{-1} / a \mathcal{O}_{K} \\ \mathrm{~N}(\lambda) \equiv-m / D}} e\left(\frac{\operatorname{tr}(\nu \lambda)}{a}\right) .
$$

Notice that our $G_{a}(m, v)$ equals $G_{a}(-m, v)$ in the notation of [Z1]. For the purposes of the present article, we need to compute $u_{0}\left(y_{1}, y_{2}, s\right)$ more explicitly.

We define a generalized divisor sum of $m$ by

$$
\sigma_{m}(s)=m^{(1-s) / 2} \sum_{d \mid m} d^{s}\left(\chi_{D}(d)+\chi_{D}\left(\frac{m}{d}\right)\right) .
$$

It satisfies the functional equation $\sigma_{m}(s)=\sigma_{m}(-s)$. If $p$ is a prime and $n \in \mathbb{Z}$, then we denote by $v_{p}(n)$ the additive $p$-adic valuation of $n$.

\section{LEMMA 2.9}

(i) If $m=m_{0} D^{\delta}$ with $\left(m_{0}, D\right)=1$, then $\sigma_{m}(s)$ has the Euler product expansion

$$
\sigma_{m}(s)=m^{(1-s) / 2}\left(1+\chi_{D}\left(m_{0}\right) D^{\delta s}\right) \prod_{\substack{p \text { prime } \\ p \mid m_{0}}} \frac{1-\chi_{D}(p)^{v_{p}\left(m_{0}\right)+1} p^{\left(v_{p}\left(m_{0}\right)+1\right) s}}{1-\chi_{D}(p) p^{s}} .
$$

(ii) If $m$ is square free and coprime to $D$, then

$$
-2 \frac{\sigma_{m}^{\prime}(-1)}{\sigma_{m}(-1)}=\sum_{p \text { prime }} \frac{p-\chi_{D}(p)}{p+\chi_{D}(p)} \log (p) .
$$




\section{Proof}

The first formula follows from the multiplicativity of $\sigma_{m}(s)$. The second can be obtained from the Euler product expansion in a straightforward way.

LEMMA 2.10

We have

$$
m^{s / 2} \sum_{a=1}^{\infty} G_{a}(m, 0) a^{-s}=\frac{\zeta(s-1)}{L\left(s, \chi_{D}\right)} \sigma_{m}(1-s) .
$$

\section{Proof}

The exponential sum $G_{a}(m, 0)$ is equal to

$$
\begin{aligned}
G_{a}(m, 0) & =\#\left\{\lambda \in \mathcal{O}_{K}(\bmod a \mathfrak{d}) ; \mathrm{N}(\lambda) \equiv-m(\bmod a D)\right\} \\
& =D^{-1} \#\left\{\lambda \in \mathcal{O}_{K}(\bmod a D) ; \mathrm{N}(\lambda) \equiv-m(\bmod a D)\right\} \\
& =D^{-1} N_{a D}(-m)
\end{aligned}
$$

with

$$
N_{b}(n)=\#\left\{\lambda \in \mathcal{O}_{K} / b \mathcal{O}_{K} ; \mathrm{N}(\lambda)=n(\bmod b)\right\},
$$

as in [Z1, p. 27]. It is easily seen that $N_{b}(n)$ is multiplicative in $b$. Hence it suffices to determine $N_{b}(n)$ for prime powers $b=p^{r}$. We get the following Euler product expansion:

$$
\begin{aligned}
\sum_{a=1}^{\infty} G_{a}(m, 0) a^{-s} & =\frac{1}{D} \sum_{a=1}^{\infty} N_{a D}(-m) a^{-s} \\
& =\frac{1}{D}\left(\sum_{r=0}^{\infty} N_{D^{1+r}}(-m) D^{-r s}\right) \prod_{\substack{p \text { prime } \\
p \neq D}}\left(\sum_{r=0}^{\infty} N_{p^{r}}(-m) p^{-r s}\right) .
\end{aligned}
$$

The function $N_{p^{r}}(n)$ can be determined explicitly by means of [Z1, Lemma 3]. By a straightforward computation, we find that the local Euler factors are equal to

$$
\begin{aligned}
\sum_{r=0}^{\infty} N_{D^{1+r}}(-m) D^{-r s} & =\frac{D}{1-D^{1-s}}\left(1+\chi_{D}\left(m_{0}\right) D^{\delta(1-s)}\right), \\
\sum_{r=0}^{\infty} N_{p^{r}}(-m) p^{-r s} & =\frac{1-\chi_{D}(p) p^{-s}}{1-p^{1-s}} \cdot \frac{1-\chi_{D}(p)^{v_{p}\left(m_{0}\right)+1} p^{\left(v_{p}\left(m_{0}\right)+1\right)(1-s)}}{1-\chi_{D}(p) p^{1-s}}
\end{aligned}
$$

for $p$ prime with $(p, D)=1$. Inserting this into (2.34), we obtain the assertion by means of Lemma 2.9. 
Hence the second term in (2.30) is equal to

$$
\frac{\pi \Gamma(s-1 / 2)^{2}}{\sqrt{D} \Gamma(2 s)}\left(\frac{4}{D}\right)^{s}\left(y_{1} y_{2}\right)^{1-s} \frac{\zeta(2 s-1)}{L\left(2 s, \chi_{D}\right)} \sigma_{m}(2 s-1) .
$$

By virtue of the functional equation

$$
L\left(2 s, \chi_{D}\right)=\left(\frac{\pi}{D}\right)^{2 s-1 / 2} \frac{\Gamma(1 / 2-s)}{\Gamma(s)} L\left(1-2 s, \chi_{D}\right)
$$

we may rewrite (2.35) in the form

$$
4^{s} \pi^{3 / 2-2 s}\left(\frac{y_{1} y_{2}}{D}\right)^{1-s} \frac{\Gamma(s-1 / 2)^{2} \Gamma(s)}{\Gamma(2 s) \Gamma(1 / 2-s)} \frac{\zeta(2 s-1)}{L\left(1-2 s, \chi_{D}\right)} \sigma_{m}(2 s-1) .
$$

Using the Legendre duplication formula, $\Gamma(s-1 / 2) \Gamma(s)=\sqrt{\pi} 2^{2-2 s} \Gamma(2 s-1)$, we finally obtain, for the second term in (2.30),

$$
-2\left(\frac{\pi^{2} y_{1} y_{2}}{D}\right)^{1-s} \frac{\Gamma(s-1 / 2)}{\Gamma(3 / 2-s)} \frac{\zeta(2 s-1)}{L\left(1-2 s, \chi_{D}\right)} \sigma_{m}(2 s-1) .
$$

In the following, we compute the first summand in (2.30). The subset

$$
S(m)=\bigcup_{\substack{\lambda \in \mathcal{D}^{-1} \\ \mathrm{~N}(\lambda)=-m / D}}\left\{\left(z_{1}, z_{2}\right) \in \mathbb{H}^{2} ; \lambda y_{1}+\lambda^{\prime} y_{2}=0\right\}
$$

of $\mathbb{H}^{2}$ is a union of hyperplanes of real codimension 1 . It is invariant under the stabilizer of the cusp $\infty$. Following the notation of [B1], we call the connected components of $\mathbb{H}^{2}-S(m)$ the Weyl chambers of discriminant $m$. For a subset $W^{\prime} \subset \mathbb{H}^{2}$ and $\lambda \in K$, we write $\left(\lambda, W^{\prime}\right)>0$ if $\lambda y_{1}+\lambda^{\prime} y_{2}>0$ for all $\left(z_{1}, z_{2}\right) \in W^{\prime}$.

Let $W \subset \mathbb{H}^{2}$ be a fixed Weyl chamber of discriminant $m$, and let $W^{\prime} \subset W$ be a nonempty subset. There are only finitely many $\lambda \in \mathfrak{d}^{-1}$ such that $\lambda>0$, $\mathrm{N}(\lambda)=-m / D$, and

$$
\left(\lambda, W^{\prime}\right)<0, \quad\left(\varepsilon_{0}^{2} \lambda, W^{\prime}\right)>0 .
$$

Denote the set of these $\lambda$ by $R\left(W^{\prime}, m\right)$. It is easily seen that $R\left(W^{\prime}, m\right)=R(W, m)$ for all nonempty subsets $W^{\prime} \subset W$. By Dirichlet's unit theorem, the set of all $\lambda \in \mathfrak{d}^{-1}$ with $\mathrm{N}(\lambda)=-m / D$ is given by

$$
\left\{ \pm \lambda \varepsilon_{0}^{2 n} ; \lambda \in R(W, m), n \in \mathbb{Z}\right\} .
$$

For $\left(z_{1}, z_{2}\right) \in W, \lambda \in R(W, m)$, and $n \in \mathbb{Z}$, we have

$$
\max \left(\left|\lambda \varepsilon_{0}^{2 n} y_{1}\right|,\left|\lambda^{\prime} \varepsilon_{0}^{2 n} y_{2}\right|\right)= \begin{cases}\lambda \varepsilon_{0}^{2 n} y_{1} & \text { if } n>0 \\ -\lambda^{\prime} \varepsilon_{0}^{\prime 2 n} y_{2} & \text { if } n \leq 0\end{cases}
$$


On $W$, we may rewrite the first summand in (2.30) as

$$
\begin{aligned}
& \frac{4 \pi}{2 s-1} \sum_{\lambda \in R(W, m)} \sum_{n \in \mathbb{Z}} \max \left(\left|\lambda \varepsilon_{0}^{2 n} y_{1}\right|,\left|\lambda^{\prime} \varepsilon_{0}^{\prime 2 n} y_{2}\right|\right)^{1-s} \min \left(\left|\lambda \varepsilon_{0}^{2 n} y_{1}\right|,\left|\lambda^{\prime} \varepsilon_{0}^{\prime 2 n} y_{2}\right|\right)^{s} \\
& =\frac{4 \pi}{2 s-1} \sum_{\lambda \in R(W, m)}\left(\sum_{n \geq 1}\left(\lambda \varepsilon_{0}^{2 n} y_{1}\right)^{1-s}\left(-\lambda^{\prime} \varepsilon_{0}^{\prime 2 n} y_{2}\right)^{s}+\sum_{n \geq 0}\left(-\lambda^{\prime} \varepsilon_{0}^{\prime-2 n} y_{2}\right)^{1-s}\left(\lambda \varepsilon_{0}^{-2 n} y_{1}\right)^{s}\right) \\
& =\frac{4 \pi}{2 s-1} \sum_{\lambda \in R(W, m)}\left(\left(\lambda y_{1}\right)^{1-s}\left(-\lambda^{\prime} y_{2}\right)^{s} \frac{\varepsilon_{0}^{2-4 s}}{1-\varepsilon_{0}^{2-4 s}}+\left(-\lambda^{\prime} y_{2}\right)^{1-s}\left(\lambda y_{1}\right)^{s} \frac{1}{1-\varepsilon_{0}^{2-4 s}}\right) .
\end{aligned}
$$

We summarize the above computations in the following theorem.

\section{THEOREM 2.11}

Let $W \subset \mathbb{H}^{2}$ be a Weyl chamber of discriminant $m$. For $\left(z_{1}, z_{2}\right) \in W$, the constant term of the Fourier expansion of $\Phi_{m}\left(z_{1}, z_{2}, s\right)$ is given by

$$
\begin{aligned}
u_{0}\left(y_{1}, y_{2}, s\right)= & 2 \zeta(2 s-1) \varphi_{m}(s)\left(\frac{\pi^{2} y_{1} y_{2}}{D}\right)^{1-s} \\
& +\frac{4 \pi}{2 s-1} \cdot \frac{1}{1-\varepsilon_{0}^{2-4 s}} \sum_{\lambda \in R(W, m)}\left(\varepsilon_{0}^{2-4 s}\left(\lambda y_{1}\right)^{1-s}\left(-\lambda^{\prime} y_{2}\right)^{s}\right. \\
& \left.+\left(-\lambda^{\prime} y_{2}\right)^{1-s}\left(\lambda y_{1}\right)^{s}\right),
\end{aligned}
$$

where

$$
\varphi_{m}(s)=-\frac{\Gamma(s-1 / 2)}{\Gamma(3 / 2-s)} \frac{1}{L\left(1-2 s, \chi_{D}\right)} \sigma_{m}(2 s-1) .
$$

As a function in $s$, the Green function $\Phi_{m}\left(z_{1}, z_{2}, s\right)$ has a simple pole at $s=1$ coming from the factor $\zeta(2 s-1)$ in the first term of $u_{0}\left(y_{1}, y_{2}, s\right)$. However, it can be regularized at this place by defining $\Phi_{m}\left(z_{1}, z_{2}\right)$ to be the constant term of the Laurent expansion of $\Phi_{m}\left(z_{1}, z_{2}, s\right)$ at $s=1$ (see [Br1, p. 66]).

Using the Laurent expansion $\zeta(2 s-1)=(1 / 2)(s-1)^{-1}-\Gamma^{\prime}(1)+O(s-1)$, we get at $s=1$ the expansion

$$
\begin{aligned}
2 \zeta(2 s-1) \varphi_{m}(s)\left(\frac{\pi^{2} y_{1} y_{2}}{D}\right)^{1-s}= & \varphi_{m}(1)(s-1)^{-1}-\varphi_{m}(1) \log \left(16 \pi^{2} y_{1} y_{2}\right) \\
& +L_{m}+O(s-1),
\end{aligned}
$$

where

$$
L_{m}=\varphi_{m}^{\prime}(1)-\varphi_{m}(1)\left(2 \Gamma^{\prime}(1)-\log (16 D)\right) .
$$


By means of the Laurent expansion of $\Gamma(s)$, one infers that $L_{m}$ is more explicitly given by

$$
L_{m}=\varphi_{m}(1)\left(2 \frac{L^{\prime}\left(-1, \chi_{D}\right)}{L\left(-1, \chi_{D}\right)}-2 \frac{\sigma_{m}^{\prime}(-1)}{\sigma_{m}(-1)}+\log (D)\right) .
$$

In later applications, it is convenient to write the regularized function $\Phi_{m}\left(z_{1}, z_{2}\right)$ as a limit. In view of (2.40), we find that

$$
\Phi_{m}\left(z_{1}, z_{2}\right)=\lim _{s \rightarrow 1}\left(\Phi_{m}\left(z_{1}, z_{2}, s\right)-\frac{\varphi_{m}(1)}{s-1}\right) .
$$

The Fourier expansion of $\Phi_{m}\left(z_{1}, z_{2}\right)$ can be deduced from (2.29) by virtue of Theorem 2.11 and (2.40). It is given by

$$
\Phi_{m}\left(z_{1}, z_{2}\right)=\lim _{s \rightarrow 1}\left(u_{0}\left(y_{1}, y_{2}, s\right)-\frac{\varphi_{m}(1)}{s-1}\right)+\sum_{\substack{v \in \mathfrak{d}^{-1} \\ v \neq 0}} u_{v}\left(y_{1}, y_{2}, 1\right) \mathbf{e}\left(v x_{1}+v^{\prime} x_{2}\right) .
$$

On a Weyl chamber $W$ of discriminant $m$, we get

$$
\begin{aligned}
\Phi_{m}\left(z_{1}, z_{2}\right)= & L_{m}-\varphi_{m}(1) \log \left(16 \pi^{2} y_{1} y_{2}\right)+4 \pi\left(\rho_{W} y_{1}+\rho_{W}^{\prime} y_{2}\right) \\
& +\sum_{\substack{v \in \mathfrak{J}^{-1} \\
v \neq 0}} u_{v}\left(y_{1}, y_{2}, 1\right) \mathbf{e}\left(v x_{1}+v^{\prime} x_{2}\right),
\end{aligned}
$$

where

$$
\rho_{W}=\frac{\varepsilon_{0}}{\operatorname{tr}\left(\varepsilon_{0}\right)} \sum_{\lambda \in R(W, m)} \lambda
$$

is the so-called Weyl vector associated with $W$ and $m$.

\section{Remark 2.12}

If we compare (2.44) with the formula given in [Br1, p. 67], we see that the quantities $L$ and $q_{0}(m)$ in [Br1] are given by $L=L_{m}-\varphi_{m}(1) \log \left(16 \pi^{2}\right)$ and $q_{0}(m)=-2 \varphi_{m}(1)$. Moreover, the Weyl vector $\rho_{W}$ in $[\mathrm{Br} 1]$ is equal to (2.45).

In order to get a Green function with a "good" arithmetic normalization, which is compatible with our normalization of the Petersson metric, we have to renormalize as follows.

\section{Definition 2.13}

We define the normalized Green function for the divisor $T(m)$ by

$$
G_{m}\left(z_{1}, z_{2}\right)=\frac{1}{2}\left(\Phi_{m}\left(z_{1}, z_{2}\right)-L_{m}\right) .
$$


According to (2.44) and [Br1, Section 3.3], we have

$$
\begin{aligned}
G_{m}\left(z_{1}, z_{2}\right)= & -\frac{\varphi_{m}(1)}{2} \log \left(16 \pi^{2} y_{1} y_{2}\right) \\
& -\log \left|\mathbf{e}\left(\rho_{W} z_{1}+\rho_{W}^{\prime} z_{2}\right) \prod_{\substack{v \in \mathcal{D}^{-1} \\
(v, W)>0 \\
\mathrm{~N}(v)=-m / D}}\left(1-\mathbf{e}\left(v z_{1}+v^{\prime} z_{2}\right)\right)\right|+o\left(z_{1}, z_{2}\right) .
\end{aligned}
$$

Here, $W$ is a Weyl chamber of discriminant $m$ and $\rho_{W}$ the corresponding Weyl vector. Moreover, $o\left(z_{1}, z_{2}\right)$ is a $\Gamma_{K, \infty}$-invariant function on $\mathbb{H}^{2}$, which defines a smooth function in the neighborhood $V_{m / D}$ of $\infty$ and vanishes at $\infty$. This describes the singularities of $G_{m}\left(z_{1}, z_{2}\right)$ near the cusp $\infty$. Analogous expansions hold at the other cusps.

We now consider $G_{m}\left(z_{1}, z_{2}\right)$ as a singular function on $\widetilde{X}(\Gamma)$. For this purpose, we also write $T(m)$ for the closure of $T(m) \subset \Gamma \backslash \mathbb{H}^{2}$ in the Baily-Borel compactification $X(\Gamma)$.

LEMMA 2.14

The divisor $T(m)$ on $X(\Gamma)$ is a $\mathbb{Q}$-Cartier divisor.

\section{Proof}

We show that there exists an integer $n$ such that $n T(m)$ is given locally as the divisor of a holomorphic function. This is clear for the restriction of $T(m)$ to $\Gamma \backslash \mathbb{H}^{2}$ because there are only finitely many singular points that are finite quotient singularities. Hence it suffices to consider $T(\mathrm{~m})$ locally at the cusps where trivializing holomorphic functions can be constructed explicitly using local Borcherds products (see $[\mathrm{BF}]$ ). For instance, for the cusp $\infty$, the function

$$
\Psi_{m}^{\infty}\left(z_{1}, z_{2}\right)=\mathbf{e}\left(\rho_{W} z_{1}+\rho_{W}^{\prime} z_{2}\right) \prod_{\substack{v \in \mathcal{O}^{-1} \\(v, W)>0 \\ \mathrm{~N}(v)=-m / D}}\left(1-\mathbf{e}\left(v z_{1}+v^{\prime} z_{2}\right)\right)
$$

is a local Borcherds product in the sense of $[\mathrm{BF}]$. There exists a positive integer $n$ such that $n \rho_{W} \in \mathfrak{d}^{-1}$. (By (2.45), one can take $n=\operatorname{tr}\left(\varepsilon_{0}\right)$.) Then $\Psi_{m}^{\infty}\left(z_{1}, z_{2}\right)^{n}$ defines a holomorphic function in a small neighborhood of $\infty$, whose divisor equals the restriction of $n T(m)$.

\section{Remark 2.15}

This lemma allows us to define the pullback $\pi^{*} T(m)$ as a $\mathbb{Q}$-Cartier divisor on $\widetilde{X}(\Gamma)$. 


\section{Remark 2.16}

Observe that $\pi^{*} T(m)$ may contain components of the exceptional divisor $\mathbf{D}_{\Gamma}$. This is actually always the case if $T(m)$ is isotropic. If $\tilde{X}\left(\Gamma_{K}\right)$ is the Hirzebruch desingularization of $X\left(\Gamma_{K}\right)$, then $\pi^{*} T(m)$ is equal to the divisor $T_{m}^{c}$ considered by Hirzebruch and Zagier in [HZ].

PROPOSITION 2.17

If $\Gamma \leq \Gamma_{K}$ is a subgroup of finite index, then

$$
\mathfrak{g}(m)=\left(-2 \partial \bar{\partial} \pi^{*} G_{m}, \pi^{*} G_{m}\right)
$$

defines a pre-log-log Green object for the $\mathbb{Q}$-divisor $\pi^{*}(T(m))$ on $\widetilde{X}(\Gamma)$.

\section{Proof}

Let $n$ be a positive integer such that $n T(m)$ is a Cartier divisor. We define a metric on the line bundle $\mathcal{O}\left(\pi^{*}(n T(m))\right)$ on $\widetilde{X}(\Gamma)$ by giving the canonical section $1_{\mathcal{O}\left(\pi^{*}(n T(m))\right)}$ the norm

$$
\left\|1_{\mathcal{O}\left(\pi^{*}(n T(m))\right)}\right\|=\exp \left(-n \pi^{*} G_{m}\right) .
$$

By [Br1, Section 3.3] and formula (38), this metric is smooth outside $\mathbf{D}_{\Gamma}$. We now show that it is a pre-log singular hermitian metric in the sense of Definition 1.20.

We consider the growth of this metric locally near points $a \in \mathbf{D}_{\Gamma}$. Here, we carry out only the case where $a$ lies above the cusp $\infty$. The other cusps are treated analogously, and the easier case where $a$ lies above an elliptic fixed point is left to the reader.

So, let $a \in \pi^{*}(\infty)$, and let $U \subset \widetilde{X}(\Gamma)$ be a small open neighborhood of $a$ such that $\pi(U) \subset V_{C}$ with $C>m / D$, as in the discussion preceding Lemma 2.1. In view of the proof of Lemma 2.14, the function $\pi^{*}\left(\Psi_{m}^{\infty}\right)^{n}$ has precisely the divisor $\pi^{*}(n T(m))$ on $U$. Thus $s=1_{\mathcal{O}\left(\pi^{*}(n T(m))\right)} / \pi^{*}\left(\Psi_{m}^{\infty}\right)^{n}$ is a trivializing section for $\mathcal{O}\left(\pi^{*}(n T(m))\right)$ on $U$. By means of (2.46), we find that

$$
\log \|s\|=n \frac{\varphi_{m}(1)}{2} \pi^{*}\left(\log \left(y_{1} y_{2}\right)\right)+\text { smooth function. }
$$

In the proof of Proposition 2.5, we already saw that $\pi^{*}\left(\log \left(y_{1} y_{2}\right)\right)$ is a pre-log-log form on $U$. Hence the assertion follows from Lemma 1.21.

\section{Notation 2.18}

To lighten the notation, we frequently drop the $\pi^{*}$. We write

$$
\mathfrak{g}(m)=\left(\omega_{m}, G_{m}\right)=\left(-2 \partial \bar{\partial} G_{m}, G_{m}\right)
$$

and also $T(m)$ instead of $\pi^{*} T(m)$. 


\section{Remark 2.19}

The Chern form $\omega_{m}$ is computed and studied in [ $\mathrm{Br} 1$, Theorem 7]. It turns out that

$$
\omega_{m}=2 \pi i \varphi_{m}(1) \omega+f\left(\varepsilon_{0} z_{1}, \varepsilon_{0}^{\prime} \bar{z}_{2}\right) \mathrm{d} z_{1} \wedge \mathrm{d} \bar{z}_{2}+f\left(\varepsilon_{0} z_{2}, \varepsilon_{0}^{\prime} \bar{z}_{1}\right) \mathrm{d} z_{2} \wedge \mathrm{d} \bar{z}_{1} .
$$

Here, $f\left(z_{1}, z_{2}\right)$ is a certain Hilbert cusp form of weight 2 for $\Gamma_{K}$, essentially the Doi-Naganuma lift of the $m$ th Poincaré series in $S_{2}^{+}\left(D, \chi_{D}\right)$. Green functions like $G_{m}$ are investigated in the context of the Weil representation in $[\mathrm{BFu}]$ and in the context of the theory of spherical functions on real Lie groups in [OT].

\section{Star products on Hilbert modular surfaces}

Here, we compute star products on Hilbert modular surfaces related to HirzebruchZagier divisors. Throughout, let $\Gamma \leq \Gamma_{K}$ be a subgroup of finite index.

\subsection{Star products for Hirzebruch-Zagier divisors}

In general, the product of a mixed growth form as $G_{m}$ and a pre-log-log form as $\omega^{2}$ need not be integrable. Therefore the following lemma, which is crucial for Theorem 3.3, is special for Hilbert modular surfaces. It seems to be related to the Koecher principle.

\section{LEMMA 3.1}

The Green function $G_{m}\left(z_{1}, z_{2}\right)$ is in $L^{1}\left(\tilde{X}(\Gamma), \omega^{2}\right)$.

\section{Proof}

By possibly replacing $\Gamma$ by a torsion-free subgroup of finite index, we may assume that $\Gamma \backslash \mathbb{H}^{2}$ is regular. Since $\widetilde{X}(\Gamma)$ is compact, it suffices to show that $G_{m}$ is locally integrable in a neighborhood of any point of $\widetilde{X}(\Gamma)$. Outside the exceptional divisor $\mathbf{D}_{\Gamma}$, this easily follows from the fact that $G_{m}$ has only logarithmic singularities along $T(m)$ on $\Gamma \backslash \mathbb{H}^{2}$. Hence we only have to show local integrability at $\mathbf{D}_{\Gamma}$. For simplicity, here, we treat the points only above the cusp $\infty$; for the other cusps, one can argue analogously.

So, let $a \in \pi^{*}(\infty)$ be a point above $\infty$. Assuming the notation of the discussion preceding Lemma 2.1, it suffices to prove that

$$
\int_{\mathbb{E}(\delta)^{2}}\left|\pi^{*}\left(G_{m}\right)\right| \pi^{*}\left(\omega^{2}\right)
$$

converges for some $\delta>0$.

By possibly replacing $\widetilde{X}(\Gamma)$ by a desingularization of $\widetilde{X}(\Gamma)$ with respect to $T(m)$, we may assume that $\pi^{*}(\infty), \pi^{*} T(m)$, and $\pi^{*}(\infty) \cup \pi^{*} T(m)$ are divisors with normal 
crossings supported on the coordinate axes of $\mathbb{E}^{2}$. By virtue of (2.46), we have

$$
\pi^{*}\left(G_{m}\right) \prec-\frac{\varphi_{m}(1)}{2} \pi^{*}\left(\log \left(y_{1} y_{2}\right)\right)-\log \left|\pi^{*}\left(\Psi_{m}^{\infty}\right)\right|
$$

on $\mathbb{E}(\delta)^{2}$. In view of the proof of Lemma 2.14, the function $\pi^{*}\left(\Psi_{m}^{\infty}\right)$ is holomorphic on $\mathbb{E}^{2}$ with divisor $\pi^{*} T(m)$. Consequently, if we introduce polar coordinates

$$
q_{j}=r_{j} e^{i \rho_{j}} \quad\left(0 \leq r_{j}<1,0 \leq \rho_{j}<2 \pi\right)
$$

on $\mathbb{E}^{2}$, we obtain by means of (2.16),

$$
\pi^{*}\left(G_{m}\right) \prec \log \left(r_{1} r_{2}\right) .
$$

We now estimate $\pi^{*}\left(\omega^{2}\right)$. As $H\left(q_{1}, q_{2}\right)$ is bounded near $(0,0)$, it follows from (2.15) that there is a small $\delta>0$ such that

$$
\begin{aligned}
& \left|\mu_{1} \log r_{1}+\mu_{2} \log r_{2}\right| \cdot\left|\mu_{1}^{\prime} \log r_{1}+\mu_{2}^{\prime} \log r_{2}\right| \\
& \quad \prec \pi^{*}\left(y_{1} y_{2}\right) \prec\left|\mu_{1} \log r_{1}+\mu_{2} \log r_{2}\right| \cdot\left|\mu_{1}^{\prime} \log r_{1}+\mu_{2}^{\prime} \log r_{2}\right|
\end{aligned}
$$

on $\mathbb{E}(\delta)^{2}$. In view of Lemma 2.1, the complex Jacobi matrix of $\pi$ at $\left(q_{1}, q_{2}\right)$ is given by

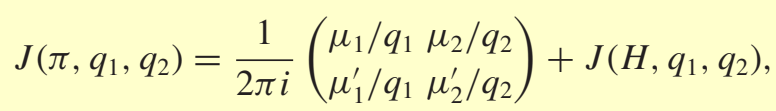

where $J\left(H, q_{1}, q_{2}\right)$ is smooth.

If $\mu_{1}$ and $\mu_{2}$ are both totally positive, we may infer that all the components of $J\left(\pi, q_{1}, q_{2}\right)$ satisfy $J\left(\pi, q_{1}, q_{2}\right)_{i, j} \prec 1 /\left(r_{1} r_{2}\right)$. Consequently, on $\mathbb{E}(\delta)^{2}$, we have

$$
\begin{aligned}
\pi^{*}\left(\omega^{2}\right) & =\pi^{*}\left(\frac{1}{8 \pi^{2}} \frac{\mathrm{d} x_{1} \mathrm{~d} y_{1}}{y_{1}^{2}} \frac{\mathrm{d} x_{2} \mathrm{~d} y_{2}}{y_{2}^{2}}\right) \\
& \prec \frac{\mathrm{d} r_{1} \mathrm{~d} r_{2} \mathrm{~d} \rho_{1} \mathrm{~d} \rho_{2}}{r_{1} r_{2}\left(\mu_{1} \log r_{1}+\mu_{2} \log r_{2}\right)^{2}\left(\mu_{1}^{\prime} \log r_{1}+\mu_{2}^{\prime} \log r_{2}\right)^{2}} .
\end{aligned}
$$

Combining the estimates (3.2) and (3.3), we see that

$$
\int_{\mathbb{E}(\delta)^{2}}\left|\pi^{*}\left(G_{m}\right)\right| \pi^{*}\left(\omega^{2}\right) \prec \int_{\mathbb{E}(\delta)^{2}} \frac{\left|\log \left(r_{1} r_{2}\right)\right| \mathrm{d} r_{1} \mathrm{~d} r_{2} \mathrm{~d} \rho_{1} \mathrm{~d} \rho_{2}}{r_{1} r_{2}\left(\mu_{1} \log r_{1}+\mu_{2} \log r_{2}\right)^{2}\left(\mu_{1}^{\prime} \log r_{1}+\mu_{2}^{\prime} \log r_{2}\right)^{2}} .
$$

In order to prove the convergence of the latter integral, it suffices to show that

$$
\int_{0}^{\delta} \int_{0}^{\delta} \frac{\left|\log r_{1}\right| \mathrm{d} r_{1} \mathrm{~d} r_{2}}{r_{1} r_{2}\left(\mu_{1} \log r_{1}+\mu_{2} \log r_{2}\right)^{2}\left(\mu_{1}^{\prime} \log r_{1}+\mu_{2}^{\prime} \log r_{2}\right)^{2}}
$$


converges. If $0 \leq \varepsilon \leq 1$, then the inequality between geometric and arithmetic mean implies that $A^{1+\varepsilon} B^{1-\varepsilon} \prec(A+B)^{2}$ uniformly for $A, B>0$. Taking into account the fact that $\mu_{1}$ and $\mu_{2}$ are totally positive, we derive that

$$
\text { (3.4) } \prec \int_{0}^{\delta} \int_{0}^{\delta} \frac{\left|\log r_{1}\right| \mathrm{d} r_{1} \mathrm{~d} r_{2}}{r_{1} r_{2}\left|\log r_{1}\right|^{2+2 \varepsilon}\left|\log r_{2}\right|^{2-2 \varepsilon}}=\int_{0}^{\delta} \frac{\mathrm{d} r_{1}}{r_{1}\left|\log r_{1}\right|^{1+2 \varepsilon}} \int_{0}^{\delta} \frac{\mathrm{d} r_{2}}{r_{2}\left|\log r_{2}\right|^{2-2 \varepsilon}} .
$$

Since the latter integrals are clearly finite for $0<\varepsilon<1 / 2$, we obtain the assertion.

If $\mu_{1}$ and $\mu_{2}$ are not both totally positive, then without any restriction we may assume that $\mu_{1} \gg 0$ and $\mu_{2}=0$. Then $\left(\log r_{1}\right)^{2} \prec \pi^{*}\left(y_{1} y_{2}\right) \prec\left(\log r_{1}\right)^{2}$ and $J\left(\pi, q_{1}, q_{2}\right) \prec 1 / r_{1}$, and the convergence of (3.1) is immediate.

\section{LEMMA 3.2}

Let $T\left(m_{1}\right), T\left(m_{2}\right), T\left(m_{3}\right)$ be Hirzebruch-Zagier divisors, and let $\kappa \in X(\Gamma)$ be a cusp or an elliptic fixed point. If $\kappa$ is a cusp, then assume, in addition, that $T\left(m_{2}\right)$ is anisotropic. If $B_{\varepsilon}(\kappa)$ denotes an $\varepsilon$-neighborhood of $\pi^{*}(\kappa) \subset \widetilde{X}(\Gamma)$, then

$$
\begin{aligned}
& \lim _{\varepsilon \rightarrow 0} \int_{\partial\left(B_{\varepsilon}(\kappa)\right)} G_{m_{1}}\left(z_{1}, z_{2}\right) \mathrm{d}^{c} G_{m_{2}}\left(z_{1}, z_{2}\right) \wedge \omega_{m_{3}}=0, \\
& \lim _{\varepsilon \rightarrow 0} \int_{\partial\left(B_{\varepsilon}(\kappa)\right)} G_{m_{2}}\left(z_{1}, z_{2}\right) \mathrm{d}^{c} G_{m_{1}}\left(z_{1}, z_{2}\right) \wedge \omega_{m_{3}}=0 .
\end{aligned}
$$

\section{Proof}

We prove the assertion only in the case where $\kappa$ is a cusp, leaving the other easier case to the reader.

By possibly interchanging $\widetilde{X}(\Gamma)$ with an embedded desingularization of $T\left(m_{1}\right)$ in $\widetilde{X}(\Gamma)$, we may assume that $\pi^{*}(\infty), \pi^{*} T\left(m_{1}\right)$, and $\pi^{*}(\infty) \cup \pi^{*} T\left(m_{1}\right)$ are divisors with normal crossings. Since $\widetilde{X}(\Gamma)$ is compact, it suffices to show that (3.5) and (3.6) hold locally. We do this only for the cusp $\infty$; at the other cusps, one can argue analogously. Let $a \in \pi^{*}(\infty)$ be a point on the exceptional divisor over $\infty$, and let $U \subset \widetilde{X}(\Gamma)$ be a small open neighborhood of $a$ such that $\left(\pi^{*} T\left(m_{2}\right)\right) \cap U=\emptyset$. After a biholomorphic change of coordinates, we may assume that $U=\mathbb{E}^{2}, a=(0,0)$, and $\pi^{*} T\left(m_{1}\right)=\operatorname{div}\left(q_{1}^{\alpha} q_{2}^{\beta}\right)$ on $U$. We assume the notation of the proof of Lemma 3.1.

Without loss of generality, it suffices to show that for some $1 \geq \delta>0$,

$$
\begin{aligned}
& \lim _{\varepsilon \rightarrow 0} \int_{\mathbb{E}(\delta) \times \partial \mathbb{E}(\varepsilon)}\left|\pi^{*}\left(G_{m_{1}}\right) \mathrm{d}^{c} \pi^{*}\left(G_{m_{2}}\right) \wedge \pi^{*}\left(\omega_{m_{3}}\right)\right|=0, \\
& \lim _{\varepsilon \rightarrow 0} \int_{\mathbb{E}(\delta) \times \partial \mathbb{E}(\varepsilon)}\left|\pi^{*}\left(G_{m_{2}}\right) \mathrm{d}^{c} \pi^{*}\left(G_{m_{1}}\right) \wedge \pi^{*}\left(\omega_{m_{3}}\right)\right|=0 .
\end{aligned}
$$


We use the local expansions of $G_{m_{1}}$ and $G_{m_{2}}$ at the cusp $\infty$ given in (2.46). On $\mathbb{E}^{2}$, we have

$$
\begin{aligned}
& \pi^{*}\left(G_{m_{1}}\right)=-\frac{\varphi_{m_{1}}(1)}{2} \pi^{*}\left(\log \left(y_{1} y_{2}\right)\right)-\log \left|q_{1}^{\alpha} q_{2}^{\beta}\right|+\text { smooth function, } \\
& \pi^{*}\left(G_{m_{2}}\right)=-\frac{\varphi_{m_{2}}(1)}{2} \pi^{*}\left(\log \left(y_{1} y_{2}\right)\right)+\text { smooth function. }
\end{aligned}
$$

Here, we have used the fact that $T\left(m_{2}\right)$ is anisotropic, which implies that $\Psi_{m_{2}}^{\infty}=1$. Furthermore, by means of (2.49), we find

$$
\pi^{*}\left(\omega_{m_{3}}\right)=2 \pi i \varphi_{m_{3}}(1) \pi^{*}(\omega)+\text { smooth differential form. }
$$

We now estimate the quantities occurring in the above boundary integrals in polar coordinates. We recall from (2.16) that

$$
\pi^{*}\left(\log \left(y_{1} y_{2}\right)\right)=\log \left(-\frac{1}{4 \pi} g_{1}\left(q_{1}, q_{2}\right)\right)+\log \left(-\frac{1}{4 \pi} g_{2}\left(q_{1}, q_{2}\right)\right),
$$

where

$$
\begin{aligned}
& g_{1}\left(q_{1}, q_{2}\right)=\mu_{1} \log \left|q_{1}\right|^{2}+\mu_{2} \log \left|q_{2}\right|^{2}-4 \pi \Im H_{1}\left(q_{1}, q_{2}\right), \\
& g_{2}\left(q_{1}, q_{2}\right)=\mu_{1}^{\prime} \log \left|q_{1}\right|^{2}+\mu_{2}^{\prime} \log \left|q_{2}\right|^{2}-4 \pi \Im H_{2}\left(q_{1}, q_{2}\right) .
\end{aligned}
$$

There is a small $1>\delta>0$ such that

$$
\mu_{1} \log r_{1}+\mu_{2} \log r_{2} \prec g_{j}\left(q_{1}, q_{2}\right) \prec \mu_{1} \log r_{1}+\mu_{2} \log r_{2}
$$

for $j=1,2$ on $\mathbb{E}\left(\delta^{2}\right)$. It follows that

$$
\begin{aligned}
& \pi^{*}\left(G_{m_{1}}\right) \prec \log r_{1}+\log r_{2}, \\
& \pi^{*}\left(G_{m_{2}}\right) \prec \log \left|\log r_{1}\right|+\log \left|\log r_{2}\right| .
\end{aligned}
$$

Moreover,

$$
\begin{aligned}
\mathrm{d}^{c} \pi^{*}\left(G_{m_{2}}\right) & \prec r_{1} \mathrm{~d} \rho_{1}+r_{2} \mathrm{~d} \rho_{2}+\mathrm{d} r_{1}+\mathrm{d} r_{2}+\mathrm{d}^{c} \pi^{*}\left(\log \left(y_{1} y_{2}\right)\right), \\
& \prec r_{1} \mathrm{~d} \rho_{1}+r_{2} \mathrm{~d} \rho_{2}+\mathrm{d} r_{1}+\mathrm{d} r_{2}+\frac{\mathrm{d}^{c} g_{1}}{g_{1}}+\frac{\mathrm{d}^{c} g_{2}}{g_{2}} \\
& \prec r_{1} \mathrm{~d} \rho_{1}+r_{2} \mathrm{~d} \rho_{2}+\mathrm{d} r_{1}+\mathrm{d} r_{2}+\frac{\mu_{1} \mathrm{~d} \rho_{1}+\mu_{2} \mathrm{~d} \rho_{2}+\mathrm{d} r_{1}+\mathrm{d} r_{2}}{\left|\mu_{1} \log r_{1}+\mu_{2} \log r_{2}\right|} .
\end{aligned}
$$


Here, " $\prec$ " is understood componentwise in the present coordinates. In the same way, we get

$$
\begin{aligned}
\mathrm{d}^{c} \pi^{*}\left(G_{m_{1}}\right) & \prec \mathrm{d} \rho_{1}+\mathrm{d} \rho_{2}+\mathrm{d} r_{1}+\mathrm{d} r_{2}+\mathrm{d}^{c} \pi^{*}\left(\log \left(y_{1} y_{2}\right)\right), \\
& \prec \mathrm{d} \rho_{1}+\mathrm{d} \rho_{2}+\mathrm{d} r_{1}+\mathrm{d} r_{2}+\frac{\mu_{1} \mathrm{~d} \rho_{1}+\mu_{2} \mathrm{~d} \rho_{2}+\mathrm{d} r_{1}+\mathrm{d} r_{2}}{\left|\mu_{1} \log r_{1}+\mu_{2} \log r_{2}\right|} .
\end{aligned}
$$

Finally, we find

$$
\begin{aligned}
\pi^{*}(\omega)= & -\operatorname{dd}^{c} \pi^{*}\left(\log \left(y_{1} y_{2}\right)\right) \\
= & \frac{\mathrm{d} g_{1} \mathrm{~d}^{c} g_{1}}{g_{1}^{2}}+\frac{\mathrm{d} g_{2} \mathrm{~d}^{c} g_{2}}{g_{2}^{2}}+\frac{4 \pi \mathrm{dd}^{c} \Im H_{1}}{g_{1}}+\frac{4 \pi \mathrm{dd}^{c} \Im H_{2}}{g_{2}} \\
\prec & \frac{\left(\mu_{1} \frac{\mathrm{d} r_{1}}{r_{1}}+\mu_{2} \frac{\mathrm{d} r_{2}}{r_{2}}+r_{1} \mathrm{~d} \rho_{1}+r_{2} \mathrm{~d} \rho_{2}\right)\left(\mu_{1} \mathrm{~d} \rho_{1}+\mu_{2} \mathrm{~d} \rho_{2}+\mathrm{d} r_{1}+\mathrm{d} r_{2}\right)}{\left(\mu_{1} \log r_{1}+\mu_{2} \log r_{2}\right)^{2}} \\
& +\frac{\left(r_{1} \mathrm{~d} \rho_{1}+r_{2} \mathrm{~d} \rho_{2}+\mathrm{d} r_{1}+\mathrm{d} r_{2}\right)^{2}}{\left|\mu_{1} \log r_{1}+\mu_{2} \log r_{2}\right|} .
\end{aligned}
$$

We now estimate the integrals in (3.7) and (3.8). We consider only the case where both $\mu_{1}$ and $\mu_{2}$ are nonzero, leaving the easier case where one of them vanishes to the reader. Only the $\mathrm{d} r_{1} \mathrm{~d} \rho_{1} \mathrm{~d} \rho_{2}$-component of the integrand gives a nonzero contribution. After a calculation, we find that in (3.7), this component is bounded by

$$
\frac{\mathrm{d} r_{1} \mathrm{~d} \rho_{1} \mathrm{~d} \rho_{2}}{r_{1}\left|\log r_{1}+\log r_{2}\right|^{2}}+\frac{r_{1}+r_{2}}{\left|\log r_{1}+\log r_{2}\right|} \mathrm{d} r_{1} \mathrm{~d} \rho_{1} \mathrm{~d} \rho_{2} .
$$

In (3.8), it is bounded by the same quantity times $\log \left|\log r_{1}\right|+\log \left|\log r_{2}\right|$. In both cases, this implies that the integrals are $O(1 / \sqrt{|\log (\varepsilon)|})$ as $\varepsilon \rightarrow 0$.

\section{THEOREM 3.3}

Let $T\left(m_{1}\right), T\left(m_{2}\right)$, and $T\left(m_{3}\right)$ be Hirzebruch-Zagier divisors such that all possible intersections on $X(\Gamma)$ among them are proper and such that $T\left(m_{2}\right)$ is anisotropic. Then

$$
\begin{aligned}
& \frac{1}{(2 \pi i)^{2}} \int_{\widetilde{X}(\Gamma)} \mathfrak{g}\left(m_{1}\right) * \mathfrak{g}\left(m_{2}\right) * \mathfrak{g}\left(m_{3}\right) \\
& =\frac{1}{(2 \pi i)^{2}} \int_{\Gamma \backslash \mathbb{H}^{2}} G_{m_{1}} \omega_{m_{2}} \wedge \omega_{m_{3}}+\frac{1}{2 \pi i} \int_{T\left(m_{1}\right)^{\prime}} \mathfrak{g}\left(m_{2}\right) * \mathfrak{g}\left(m_{3}\right) .
\end{aligned}
$$

Here, $T\left(m_{1}\right)^{\prime}$ denotes the strict transform of the divisor $T\left(m_{1}\right)$ on $\widetilde{X}(\Gamma)$. 


\section{Proof}

Let $\mathbf{D}_{1}$ be the sum of the connected components of $\mathbf{D}_{\Gamma}$, which have nonempty intersection with $T\left(m_{1}\right)$ on $\widetilde{X}(\Gamma)$. To ease the notation, we put $\mathfrak{g}_{2,3}=\mathfrak{g}\left(m_{2}\right) * \mathfrak{g}\left(m_{3}\right)$. Since $T\left(m_{2}\right)$ and $T\left(m_{3}\right)$ may intersect at elliptic fixed points, in general they do not intersect properly on $\widetilde{X}(\Gamma)$. Therefore $\mathfrak{g}_{2,3}=\left(-2 \partial \bar{\partial} g_{2,3}, g_{2,3}\right)$ is a weak pre-log-log Green object for the zero cycle $T\left(m_{2}\right) \cdot T\left(m_{3}\right)$ with support $T\left(m_{2}\right) \cap T\left(m_{3}\right)$, which has empty intersection with $\mathbf{D}_{1}$. Thus the assumptions of Theorem 1.14 are satisfied. Consequently,

$$
\begin{aligned}
& \frac{1}{(2 \pi i)^{2}} \int_{\widetilde{X}(\Gamma)} \mathfrak{g}\left(m_{1}\right) * \mathfrak{g}_{2,3} \\
& =\lim _{\varepsilon \rightarrow 0}\left(\frac{1}{(2 \pi i)^{2}} \int_{\widetilde{X}(\Gamma) \backslash B_{\varepsilon}\left(\mathbf{D}_{\Gamma}\right)} G_{m_{1}} \wedge-2 \partial \bar{\partial} g_{2,3}\right. \\
& \left.\quad-\frac{2}{2 \pi i} \int_{\partial\left(B_{\varepsilon}\left(\mathbf{D}_{1}\right)\right)} g_{2,3} \wedge \mathrm{d}^{c} G_{m_{1}}-G_{m_{1}} \wedge \mathrm{d}^{c} g_{2,3}\right) \\
& \quad+\frac{1}{2 \pi i} \int_{\overline{T\left(m_{1}\right) \backslash\left(T\left(m_{1}\right) \cap \mathbf{D}_{1}\right)}} g_{2,3} .
\end{aligned}
$$

Since $-2 \partial \bar{\partial} g_{2,3}=\omega_{m_{2}} \wedge \omega_{m_{3}}$ and $\omega_{m_{2}} \wedge \omega_{m_{3}} \prec \omega^{2}$, we obtain by Lemma 3.1, for the first integral in question,

$$
\lim _{\varepsilon \rightarrow 0}\left(\int_{\widetilde{X}(\Gamma) \backslash B_{\varepsilon}\left(\mathbf{D}_{\Gamma}\right)} G_{m_{1}} \wedge-2 \partial \bar{\partial} g_{2,3}\right)=\int_{\Gamma \backslash \mathbb{H}^{2}} G_{m_{1}} \omega_{m_{2}} \wedge \omega_{m_{2}} .
$$

We now show that the integrals along the boundary vanish in the limit. Recall that by (1.6), a representative of $\mathfrak{g}_{2,3}$ is given by the pair

$$
\left(\omega_{m_{2}} \wedge \omega_{m_{3}}, \sigma_{3,2} G_{m_{2}} \cdot \omega_{m_{3}}-2 \partial \bar{\partial}\left(\sigma_{2,3} G_{m_{2}}\right) \cdot G_{m_{3}}\right)
$$

here, $\sigma_{2,3}:=\sigma_{T\left(m_{2}\right), T\left(m_{3}\right)}$ and $\sigma_{3,2}$ form a partition of unity as in (1.5). Because $T\left(m_{2}\right) \cap$ $T\left(m_{3}\right) \cap E_{j}=\emptyset$ for all irreducible components $E_{j}$ of $\mathbf{D}_{1}$, we may choose $\sigma_{2,3}$ so that

$$
\left.g_{2,3}\right|_{\partial\left(B_{\epsilon}\left(E_{j}\right)\right)}= \begin{cases}G_{m_{3}} \omega_{m_{2}} & \text { if } T\left(m_{2}\right) \cap E_{j} \neq \emptyset, \\ G_{m_{2}} \omega_{m_{3}} & \text { if } T\left(m_{2}\right) \cap E_{j}=\varnothing .\end{cases}
$$

In particular, by our assumption on $T\left(m_{2}\right)$, if $E_{j}$ lies above a cusp, then the second case applies. Because $\omega_{m_{j}} \prec \omega$ for $j=2,3$, we find by Lemma 3.2,

$$
\lim _{\varepsilon \rightarrow 0}\left(\int_{\partial\left(B_{\varepsilon}\left(\mathbf{D}_{1}\right)\right)} g_{2,3} \wedge \mathrm{d}^{c} G_{m_{1}}-G_{m_{1}} \wedge \mathrm{d}^{c} g_{2,3}\right)=0 .
$$


Finally, since $T\left(m_{1}\right)^{\prime}=\overline{T\left(m_{1}\right) \backslash\left(T\left(m_{1}\right) \cap \mathbf{D}_{1}\right)}$, we observe that

$$
\frac{1}{2 \pi i} \int_{\overline{T\left(m_{1}\right) \backslash\left(T\left(m_{1}\right) \cap \mathbf{D}_{1}\right)}} g_{2,3}=\frac{1}{2 \pi i} \int_{T\left(m_{1}\right)^{\prime}} \mathfrak{g}\left(m_{2}\right) * \mathfrak{g}\left(m_{3}\right) .
$$

Notice that in the above star product, the components of the desingularization of the divisor $T\left(m_{1}\right)$ do not contribute. In other words, this star product is independent of the choice we made in the desingularization and depends only on the Baily-Borel compactification $X(\Gamma)$.

\section{Remark 3.4}

The formula for the star product in Theorem 3.3 agrees with the formula by Gillet and Soule for the star product of Green currents when formally applied to the Baily-Borel compactification $X(\Gamma)$ that is a singular space.

\section{Remark 3.5}

Since $T(m)$ and $\mathfrak{g}(m)$ are invariant under the full Hilbert modular group $\Gamma_{K}$, we clearly have

$$
\int_{\widetilde{X}(\Gamma)} \mathfrak{g}\left(m_{1}\right) * \mathfrak{g}\left(m_{2}\right) * \mathfrak{g}\left(m_{3}\right)=\left[\Gamma_{K}: \Gamma\right] \int_{\widetilde{X}_{\left(\Gamma_{K}\right)}} \mathfrak{g}\left(m_{1}\right) * \mathfrak{g}\left(m_{2}\right) * \mathfrak{g}\left(m_{3}\right) .
$$

\subsection{Integrals of Green functions}

The purpose of this section is to compute the first integral in (3.9) in the case of $\omega_{m_{2}}=\omega_{m_{3}}=2 \pi i \omega$. Recall our normalization (2.14) of the invariant Kähler form $\omega$. In addition, we put

$$
\eta_{1}=\frac{1}{4 \pi} \frac{\mathrm{d} x_{1} \mathrm{~d} y_{1}}{y_{1}^{2}}, \quad \eta_{2}=\frac{1}{4 \pi} \frac{\mathrm{d} x_{2} \mathrm{~d} y_{2}}{y_{2}^{2}} .
$$

We consider the quadratic space $V$ and the lattices $L=L\left(\mathcal{O}_{K}\right), L^{\prime}=L^{\prime}\left(\mathcal{O}_{K}\right)$ defined at the beginning of Section 2.3. We put $W=\left(\begin{array}{cc}0 & -1 \\ 1 & 0\end{array}\right)$. The Hilbert modular

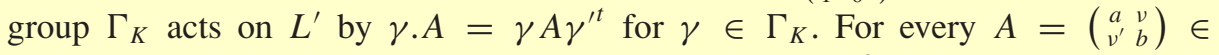
$L^{\prime}$, the graph $\{(W A z, z) ; z \in \mathbb{H}\}$ defines a divisor in $\mathbb{H}^{2}$ given by the equation $a z_{1} z_{2}+v z_{1}+v^{\prime} z_{2}+b=0$. The stabilizer $\Gamma_{K, A}$ of $A$ under the action of $\Gamma_{K}$ can be viewed as an arithmetic subgroup of $\mathrm{SL}_{2}(\mathbb{R})$ with finite covolume (see [Z1, Section 1], [G, Chapter 5.1]). By reduction theory, the subset

$$
L_{m}^{\prime}=\left\{A \in L^{\prime} ; \operatorname{det} A=\frac{m}{D}\right\}
$$


of elements with norm $m / D$ decomposes into finitely many $\Gamma_{K}$-orbits. The divisor $T(m)$ on $\Gamma_{K} \backslash \mathbb{H}^{2}$ is given by

$$
T(m) \cong \bigcup_{A \in \Gamma_{K} \backslash L_{m}^{\prime} /\{ \pm 1\}} \Gamma_{K, A} \backslash \mathbb{H} .
$$

Here, $\{ \pm 1\}$ acts on $L_{m}^{\prime}$ by scalar multiplication.

We may rewrite $\Phi_{m}\left(z_{1}, z_{2}, s\right)$ using this splitting. For $A=\left(\begin{array}{cc}a & v \\ v^{\prime} & b\end{array}\right) \in L_{m}^{\prime}$, we define

$$
d_{A}\left(z_{1}, z_{2}\right)=1+\frac{\left|z_{1}-W A z_{2}\right|^{2}}{2 \Im\left(z_{1}\right) \Im\left(W A z_{2}\right)}=1+\frac{\left|a z_{1} z_{2}+v z_{1}+v^{\prime} z_{2}+b\right|^{2}}{2 y_{1} y_{2} m / D} .
$$

If $\gamma \in \Gamma_{K}$, we have

$$
d_{\gamma \cdot A}\left(z_{1}, z_{2}\right)=d_{A}\left(\gamma^{t} z_{1}, \gamma^{\prime t} z_{2}\right)
$$

This follows from the fact that $\left|z_{1}-z_{2}\right|^{2} /\left(2 y_{1} y_{2}\right)$ is a point-pair invariant; that is, it depends only on the hyperbolic distance of $z_{1}$ and $z_{2}$. Consequently,

$$
\Phi_{m}\left(z_{1}, z_{2}, s\right)=\sum_{A \in L_{m}^{\prime}} Q_{s-1}\left(d_{A}\left(z_{1}, z_{2}\right)\right)=\sum_{A \in \Gamma_{K} \backslash L_{m}^{\prime}} \sum_{\gamma \in \Gamma_{K, A} \backslash \Gamma_{K}} Q_{s-1}\left(d_{A}\left(\gamma z_{1}, \gamma^{\prime} z_{2}\right)\right) .
$$

PROPOSITION 3.6

The integral

$$
\int_{\Gamma_{K} \backslash \mathbb{H}^{2}}\left|\Phi_{m}\left(z_{1}, z_{2}, s\right)\right| \omega^{2}
$$

converges for all $s \in \mathbb{C}$ with $\Re(s)>1$.

\section{Proof}

According to (3.14), we have the formal identity

$$
\begin{aligned}
\int_{\Gamma_{K} \backslash \mathbb{H}^{2}}\left|\Phi_{m}\left(z_{1}, z_{2}, s\right)\right| \omega^{2} & =\sum_{A \in \Gamma_{K} \backslash L_{m}^{\prime}} \int_{\Gamma_{K} \backslash \mathbb{H}^{2}} \sum_{\gamma \in \Gamma_{K, A} \backslash \Gamma_{K}}\left|Q_{s-1}\left(d_{A}\left(\gamma z_{1}, \gamma^{\prime} z_{2}\right)\right)\right| \omega^{2} \\
& =\sum_{A \in \Gamma_{K} \backslash L_{m}^{\prime}} \int_{\Gamma_{K, A} \backslash \mathbb{H}^{2}}\left|Q_{s-1}\left(d_{A}\left(z_{1}, z_{2}\right)\right)\right| \omega^{2} \\
& =2 \sum_{A \in \Gamma_{K} \backslash L_{m}^{\prime}} \int_{z_{2} \in \Gamma_{K, A} \backslash \mathbb{H}} \int_{z_{1} \in \mathbb{H}}\left|Q_{s-1}\left(d_{A}\left(z_{1}, z_{2}\right)\right)\right| \eta_{1} \eta_{2} .
\end{aligned}
$$


By a standard Fubini-type lemma on integrals over Poincaré series (see, e.g., [Fr3, Appendix 2, Theorem 7]), the integral on the left-hand side converges (and equals the right-hand side) if the latter integral on the right-hand side converges. Thus it suffices to prove that

$$
\int_{z_{2} \in \Gamma_{K, A} \backslash \mathbb{H}} \int_{z_{1} \in \mathbb{H}}\left|Q_{s-1}\left(d_{A}\left(z_{1}, z_{2}\right)\right)\right| \eta_{1} \eta_{2}
$$

converges. We notice that the inner integral actually does not depend on $z_{2}$ and $A$. Using the fact that $\left|z_{1}-z_{2}\right|^{2} /\left(2 y_{1} y_{2}\right)$ is a point-pair invariant and the invariance of $\eta_{1}$, we find that (3.16) is equal to

$$
\int_{z_{2} \in \Gamma_{K, A} \backslash \mathbb{H}} \int_{z_{1} \in \mathbb{H}}\left|Q_{s-1}\left(1+\frac{\left|z_{1}-i\right|^{2}}{2 \Im z_{1} \Im i}\right)\right| \eta_{1} \eta_{2} .
$$

This integral is obviously bounded by

$$
\operatorname{vol}\left(\Gamma_{K, A} \backslash \mathbb{H}\right) \int_{z_{1} \in \mathbb{H}}\left|Q_{s-1}\left(1+\frac{\left|z_{1}-i\right|^{2}}{2 y_{1}}\right)\right| \eta_{1} .
$$

That the latter integral is finite for $\Re(s)>1$ is a well-known fact (see, e.g., [L, Chapter 14, Section 3]).

\section{LEMMA 3.7}

Let $h: \mathbb{H} \rightarrow \mathbb{C}$ be a bounded eigenfunction of the hyperbolic Laplacian $\Delta_{1}$ with eigenvalue $\lambda$. Then for $s \in \mathbb{C}$ with $\Re(s)>1$, we have

$$
\int_{\mathbb{H}} Q_{s-1}\left(1+\frac{\left|z_{1}-z_{2}\right|^{2}}{2 y_{1} y_{2}}\right) h\left(z_{1}\right) \eta_{1}=\frac{1 / 2}{s(s-1)-\lambda} h\left(z_{2}\right) .
$$

\section{Proof}

This statement is well known. It can be proved using the Green formula (see, e.g., [I, Chapter 1.9]; notice the different normalization there).

\section{THEOREM 3.8}

Let $f: \Gamma_{K} \backslash \mathbb{H}^{2} \rightarrow \mathbb{C}$ be a bounded eigenfunction of the Laplacian $\Delta_{1}$ (or $\Delta_{2}$ ) with eigenvalue $\lambda$. Then for $s \in \mathbb{C}$ with $\Re(s)>1$, we have

$$
\int_{\Gamma_{K} \backslash \mathbb{H}^{2}} \Phi_{m}\left(z_{1}, z_{2}, s\right) f\left(z_{1}, z_{2}\right) \omega^{2}=\frac{1}{s(s-1)-\lambda} \int_{T(m)} f\left(z_{1}, z_{2}\right) \omega .
$$

\section{Proof}

Since $\Phi_{m}\left(z_{1}, z_{2}, s\right)$ is invariant under $\left(z_{1}, z_{2}\right) \mapsto\left(z_{2}, z_{1}\right)$, we may assume that $f$ is an eigenfunction of $\Delta_{1}$ with eigenvalue $\lambda$. 
First, we notice that the integral $I$ on the left-hand side converges by Proposition 3.6. Similarly, as in the proof of Proposition 3.6, we rewrite it as

$$
\begin{aligned}
I & =\int_{\Gamma_{K} \backslash \mathbb{H}^{2}} \sum_{A \in \Gamma_{K} \backslash L_{m}^{\prime}} \sum_{\gamma \in \Gamma_{K, A} \backslash \Gamma_{K}} Q_{s-1}\left(d_{A}\left(\gamma z_{1}, \gamma^{\prime} z_{2}\right)\right) f\left(z_{1}, z_{2}\right) \omega^{2} \\
& =2 \sum_{A \in \Gamma_{K} \backslash L_{m}^{\prime}} \int_{z_{2} \in \Gamma_{K, A} \backslash \mathbb{H}} \int_{z_{1} \in \mathbb{H}} Q_{s-1}\left(1+\frac{\left|z_{1}-W A z_{2}\right|^{2}}{2 \Im\left(z_{1}\right) \Im\left(W A z_{2}\right)}\right) f\left(z_{1}, z_{2}\right) \eta_{1} \eta_{2} .
\end{aligned}
$$

Here, the inner integral can be computed by means of Lemma 3.7. We obtain

$$
\begin{aligned}
I & =\frac{2}{s(s-1)-\lambda} \sum_{A \in \Gamma_{K} \backslash L_{m}^{\prime} /\{ \pm 1\}} \int_{z_{2} \in \Gamma_{K, A} \backslash \mathbb{H}} f\left(W A z_{2}, z_{2}\right) \eta_{2} \\
& =\frac{1}{s(s-1)-\lambda} \int_{T(m)} f\left(z_{1}, z_{2}\right) \omega .
\end{aligned}
$$

This concludes the proof of the theorem.

COROLLARY 3.9

(i) If $s \in \mathbb{C}$ with $\Re(s)>1$, then

$$
\int_{\Gamma_{K} \backslash \mathbb{H}^{2}} \Phi_{m}\left(z_{1}, z_{2}, s\right) \omega^{2}=\frac{2}{s(s-1)} \operatorname{vol}(T(m)) .
$$

(ii) The volume of $T(m)$ with respect to the invariant volume form $\frac{\mathrm{d} x \mathrm{~d} y}{4 \pi y^{2}}$ on $\mathbb{H}$ equals

$$
\operatorname{vol}(T(m))=\frac{1}{2} \zeta_{K}(-1) \varphi_{m}(1)=\frac{1}{24} \sigma_{m}(-1)
$$

(iii) We have

$$
\begin{aligned}
& \int_{\Gamma_{K} \backslash \mathbb{H}^{2}} G_{m}\left(z_{1}, z_{2}\right) \omega^{2} \\
& \quad=-\operatorname{vol}(T(m))\left(2 \frac{L^{\prime}\left(-1, \chi_{D}\right)}{L\left(-1, \chi_{D}\right)}-2 \frac{\sigma_{m}^{\prime}(-1)}{\sigma_{m}(-1)}+1+\log (D)\right) .
\end{aligned}
$$

\section{Proof}

If we use Theorem 3.8 with $f=1$, we get

$$
\begin{aligned}
\int_{\Gamma_{K} \backslash \mathbb{H}^{2}} \Phi_{m}\left(z_{1}, z_{2}, s\right) \omega^{2} & =\frac{1}{s(s-1)} \int_{T(m)} \omega=\frac{2}{s(s-1)} \operatorname{vol}(T(m)) \\
& =\frac{2 \operatorname{vol}(T(m))}{s-1}-2 \operatorname{vol}(T(m))+O(s-1) .
\end{aligned}
$$


By (2.19), we have, in addition,

$$
\int_{\Gamma_{K} \backslash \mathbb{H}^{2}} \frac{\varphi_{m}(1)}{s-1} \omega^{2}=\frac{1}{s-1} \zeta_{K}(-1) \varphi_{m}(1) .
$$

Since $\Phi_{m}\left(z_{1}, z_{2}\right)=\lim _{s \rightarrow 1}\left(\Phi_{m}\left(z_{1}, z_{2}, s\right)-\varphi_{m}(1) /(s-1)\right)$ is regular and integrable at $s=1$, we derive the second claim by comparing residues in the latter two equalities. The third claim follows from Definition 2.13 and (2.42) by comparing the constant terms.

Notice that our normalization of $\operatorname{vol} T(m)$ equals $-1 / 2$ times the normalization of [HZ] and [G, Chapter 5.5]. In particular, here, the volume of $T(1) \cong \Gamma(1) \backslash \mathbb{H}$ is $1 / 12$.

\subsection{Star products on isotropic Hirzebruch-Zagier divisors}

For the rest of this section, we assume that $p$ is a prime that is split in $\mathcal{O}_{K}$ or $p=1$. Let $\mathfrak{p}$ be a prime ideal of $\mathcal{O}_{K}$ above $p$. There are a fractional ideal $\mathfrak{c}$ and a totally positive $\lambda \in K$ such that $\mathfrak{p}=\lambda \mathfrak{c}^{2}$. We fix a matrix $M \in\left(\begin{array}{ll}\mathfrak{c}^{-1} & \mathfrak{c}^{-1} \\ \mathfrak{c} & \mathfrak{c}\end{array}\right) \cap \operatorname{SL}_{2}(K)$.

It is well known that the isotropic Hirzebruch-Zagier divisor $T(p) \subset \Gamma_{K} \backslash \mathbb{H}^{2}$ is irreducible (see [G, Chapter 5.1] and [HZ]). It may have points of self-intersection, and its normalization is isomorphic to the noncompact modular curve $Y_{0}(p)=\Gamma_{0}(p) \backslash \mathbb{H}$. The normalization of the closure of $T(p)$ in $X\left(\Gamma_{K}\right)$ is isomorphic to the compact modular curve $X_{0}(p)$, the standard compactification of $Y_{0}(p)$ (for basic facts on integral models of $X_{0}(p)$, the line bundle of modular forms on it, and the normalization of the corresponding Petersson metric, we refer to [Kü2]).

We now describe how $T(p)$ can be parametrized; later, in Proposition 5.11, we give a modular description of this map. On the Hilbert modular surface $X\left(\Gamma\left(\mathcal{O}_{K} \oplus \mathfrak{p}\right)\right)$, the Hirzebruch-Zagier divisor $T_{\mathfrak{p}}(p)$ is simply given by the diagonal. More precisely, the assignment $\tau \mapsto(\tau, \tau)$ induces a morphism of degree 1 ,

$$
X_{0}(p) \longrightarrow X\left(\Gamma\left(\mathcal{O}_{K} \oplus \mathfrak{p}\right)\right)
$$

whose image is $T_{\mathfrak{p}}(p)$. In fact, the vector $\left(\begin{array}{lr}0 & p / \sqrt{D} \\ -p / \sqrt{D} & 0\end{array}\right) \in L^{\prime}(\mathfrak{p})$ has determinant $p^{2} / D$. Since $T_{\mathfrak{p}}(p)$ is irreducible, the image of the diagonal in $X\left(\Gamma\left(\mathcal{O}_{K} \oplus \mathfrak{p}\right)\right)$ is $T_{\mathfrak{p}}(p)$. Moreover, it is easily checked that the stabilizer in $\Gamma\left(\mathcal{O}_{K} \oplus \mathfrak{p}\right)$ of the diagonal in $\mathbb{H}^{2}$ is equal to $\Gamma_{0}(p)$. The cusp $\infty$ (resp., 0$)$ of $X_{0}(p)$ is mapped to the cusp $\infty$ (resp., 0$)$ of $X\left(\Gamma\left(\mathcal{O}_{K} \oplus \mathfrak{p}\right)\right)$.

PROPOSITION 3.10

The map $\mathbb{H} \rightarrow \mathbb{H}^{2}, \tau \mapsto M^{-1}\left(\begin{array}{cc}\lambda & 0 \\ 0 & 1\end{array}\right)(\tau, \tau)$ induces a morphism

$$
\varphi: X_{0}(p) \longrightarrow X\left(\Gamma_{K}\right)
$$

which is generically one-to-one and whose image equals $T(p)$. The cusp $\infty$ (resp., 0$)$ of $X_{0}(p)$ is mapped to the cusp $\mathfrak{c}$ (resp., $\left.\mathfrak{c}^{-1}\right)$ of $X\left(\Gamma_{K}\right)$. 


\section{Proof}

The map $\varphi$ is given by the commutative diagram

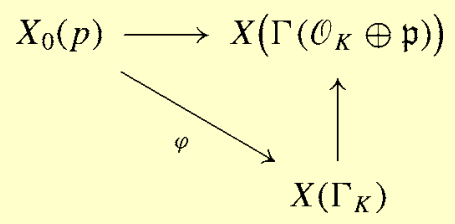

where the horizontal arrow is given by (3.20) and the vertical arrow (which is an isomorphism) by (2.5). The properties of the latter two maps imply the assertion.

It is easily seen that the pullback of the Kähler form $\omega$ equals $2 \frac{\mathrm{d} x \mathrm{~d} y}{4 \pi y^{2}}$.

For the next proposition, we recall that the Fricke involution $W_{p}$ on the space of modular forms of weight $k$ for $\Gamma_{0}(p)$ is defined by $f(z) \mapsto\left(\left.f\right|_{k} W_{p}\right)(z)=$ $p^{k / 2} z^{-k} f(-1 / p z)$. Moreover, we define the Petersson slash operator for Hilbert modular forms in weight $k$ by

$$
\left(\left.F\right|_{k} \gamma\right)\left(z_{1}, z_{2}\right)=\mathrm{N}(\operatorname{det}(\gamma))^{k / 2}\left(c z_{1}+d\right)^{-k}\left(c^{\prime} z_{2}+d^{\prime}\right)^{-k} F\left(\gamma z_{1}, \gamma^{\prime} z_{2}\right)
$$

for $\gamma=\left(\begin{array}{ll}a & b \\ c & d\end{array}\right) \in \mathrm{GL}_{2}^{+}(K)$.

PROPOSITION 3.11

(i) If $F$ is a Hilbert modular form of weight $k$ for $\Gamma_{K}$, then its pullback

$$
\left(\varphi^{*} F\right)(\tau)=\left(\left.F\right|_{k} M^{-1}\left(\begin{array}{ll}
\lambda & 0 \\
0 & 1
\end{array}\right)\right)(\tau, \tau)
$$

is a modular form of weight $2 k$ for the group $\Gamma_{0}(p)$.

(ii) If $F$ is, in addition, holomorphic and has the Fourier expansions

$$
\begin{aligned}
\left.F\right|_{k} M^{-1}\left(\begin{array}{cc}
\lambda & 0 \\
0 & 1
\end{array}\right) & =\sum_{\nu \in \mathfrak{p} \mathfrak{d}^{-1}} a_{v} \mathbf{e}\left(v z_{1}+v^{\prime} z_{2}\right), \\
\left.F\right|_{k} M^{\prime-1}\left(\begin{array}{cc}
\lambda^{\prime} & 0 \\
0 & 1
\end{array}\right) & =\sum_{\nu \in \mathfrak{p}^{\prime} \mathfrak{d}^{-1}} b_{\nu} \mathbf{e}\left(v z_{1}+v^{\prime} z_{2}\right),
\end{aligned}
$$

at the cusps $\mathfrak{c}$ and $\mathfrak{c}^{\prime}$, respectively, then the Fourier expansions of $\varphi^{*} F$ at $\infty$ and 0 are given by

$$
\begin{aligned}
\varphi^{*} F & =\sum_{n=0}^{\infty} \sum_{\substack{\nu \in \mathfrak{p d}^{-1} \\
\operatorname{tr}(v)=n}} a_{v} \mathbf{e}(n \tau), \\
\left.\left(\varphi^{*} F\right)\right|_{k} W_{p} & =\sum_{n=0}^{\infty} \sum_{\substack{\nu \in \mathfrak{p}^{\prime} \mathfrak{d}^{-1} \\
\operatorname{tr}(v)=n}} b_{\nu} \mathbf{e}(n \tau) .
\end{aligned}
$$


(iii) For the Petersson metric, we have

$$
\varphi^{*}\left(\log \|F\|_{\text {Pet }}\right)=\log \left(\left\|\varphi^{*} F\right\|_{\text {Pet }}\right)
$$

\section{Remark 3.12}

Proposition 3.11 implies, in particular, that Hilbert modular forms with rational Fourier coefficients are mapped to modular forms for $\Gamma_{0}(p)$ with rational Fourier coefficients. This shows that $\varphi$ is actually defined over $\mathbb{Q}$ (see Proposition 5.5).

We now compute certain star products of pullbacks of Hilbert modular forms via $\varphi$.

THEOREM 3.13

Let $F, G$ be Hilbert modular forms of weight $k$ with rational Fourier coefficients. Assume that all possible intersections on $X\left(\Gamma_{K}\right)$ of $T(p), \operatorname{div}(F), \operatorname{div}(G)$ are proper, and assume that $F$ does not vanish at the cusps $\mathfrak{c}$ and $\mathfrak{c}^{-1}$ of $\Gamma_{K}$. Then

$$
\begin{aligned}
& \frac{1}{2 \pi i} \int_{X_{0}(p)} \varphi^{*} \mathfrak{g}(F) * \varphi^{*} \mathfrak{g}(G) \\
& \quad=-(2 k)^{2} \operatorname{vol}(T(p))\left(\frac{\zeta^{\prime}(-1)}{\zeta(-1)}+\frac{1}{2}\right)-\left(\operatorname{div}\left(\varphi^{*} F\right), \operatorname{div}\left(\varphi^{*} G\right)\right)_{\mathscr{X}_{0}(p), \text { fin }}
\end{aligned}
$$

Here, $\left(\operatorname{div}\left(\varphi^{*} F\right), \operatorname{div}\left(\varphi^{*} G\right)\right)_{\mathscr{X}_{0}(p) \text {, fin }}$ denotes the intersection number at the finite places on the minimal regular model $\mathscr{X}_{0}(p)$ of $X_{0}(p)$ of the divisors associated with the sections of the line bundle of modular forms corresponding to $\varphi^{*} F$ and $\varphi^{*} G$ (see [Kü2]).

\section{Proof}

Because of (3.23), $\varphi^{*} \mathfrak{g}(F)=\mathfrak{g}\left(\varphi^{*} F\right)$. Thus, on $X_{0}(p)$, we have

$$
\varphi^{*} \mathfrak{g}(F) * \varphi^{*} \mathfrak{g}(G)=\mathfrak{g}\left(\varphi^{*} F\right) * \mathfrak{g}\left(\varphi^{*} G\right) .
$$

By assumption, $\varphi^{*} F, \varphi^{*} G$ are modular forms for $\Gamma_{0}(p)$ of weight $2 k$ with rational Fourier expansions, and $\varphi^{*} F$ does not vanish at the cusps. They determine sections of the line bundle of modular forms of weight $2 k$ on $\mathscr{X}_{0}(p)$. Notice that on $X_{0}(p)$, we have $\operatorname{div}\left(\varphi^{*} F\right) \cap \operatorname{div}\left(\varphi^{*} G\right)=\emptyset$. By the compatibility with the arithmetic intersection product of [Kü2], the left-hand side of (3.24) equals the generalized arithmetic intersection number $\left\langle\varphi^{*} F, \varphi^{*} G\right\rangle_{\infty}$ at the infinite place given in [Kü1, Lemma 3.9]. Hence we have

$$
\frac{1}{2 \pi i} \int_{X_{0}(p)} \varphi^{*} \mathfrak{g}(F) * \varphi^{*} \mathfrak{g}(G)=\left\langle\varphi^{*} F, \varphi^{*} G\right\rangle_{\infty}
$$


Now, the claim follows from [Kü1, Corollary 6.2]; that is,

$(2 k)^{2}(p+1)\left(\frac{1}{2} \zeta(-1)+\zeta^{\prime}(-1)\right)=\left(\operatorname{div}\left(\varphi^{*} F\right), \operatorname{div}\left(\varphi^{*} G\right)\right)_{\mathscr{X}_{0}(p), \operatorname{fin}}+\left\langle\varphi^{*} F, \varphi^{*} G\right\rangle_{\infty}$, using the identity $\operatorname{vol} T(p)=\operatorname{vol} X_{0}(p)=-\zeta(-1)\left[\Gamma(1): \Gamma_{0}(p)\right]=(p+1) / 12$.

\subsection{Star products on Hilbert modular surfaces}

We combine the results of the previous sections to compute star products on Hilbert modular surfaces.

\section{THEOREM 3.14}

Let $p$ be a prime that is split in $\mathcal{O}_{K}$ or $p=1$, and let $F, G$ be Hilbert modular forms of weight $k$ with rational Fourier coefficients. Assume that all possible intersections on $X\left(\Gamma_{K}\right)$ of $T(p), \operatorname{div}(F), \operatorname{div}(G)$ are proper, and assume that $F$ does not vanish at any cusp of $\Gamma_{K}$. Then

$$
\begin{aligned}
& \frac{1}{(2 \pi i)^{2}} \int_{\widetilde{X}\left(\Gamma_{K}\right)} \mathfrak{g}(p) * \mathfrak{g}(F) * \mathfrak{g}(G) \\
& =-k^{2} \operatorname{vol}(T(p))\left(2 \frac{\zeta_{K}^{\prime}(-1)}{\zeta_{K}(-1)}+2 \frac{\zeta^{\prime}(-1)}{\zeta(-1)}+3+\log (D)\right) \\
& \quad-k^{2} \operatorname{vol}(T(p)) \frac{p-1}{p+1} \log (p)-\left(\operatorname{div}\left(\varphi^{*} F\right), \operatorname{div}\left(\varphi^{*} G\right)\right)_{X_{0}(p), \operatorname{fin}} .
\end{aligned}
$$

\section{Proof}

The logarithm of the Petersson norm of a Hilbert modular form satisfies along $\mathbf{D}_{\Gamma}$ the same bounds as the Green functions $G_{m}$. Hence we may calculate the star product in question by means of the formula of Theorem 3.3:

$$
\frac{1}{(2 \pi i)^{2}} \int_{\widetilde{X}_{\left(\Gamma_{K}\right)}} \mathfrak{g}(p) * \mathfrak{g}(F) * \mathfrak{g}(G)=k^{2} \int_{\Gamma_{K} \backslash \mathbb{H}^{2}} G_{p} \omega^{2}+\frac{1}{2 \pi i} \int_{T(p)^{\prime}} \mathfrak{g}(F) * \mathfrak{g}(G) .
$$

By Corollary 3.9(iii) and (2.33), the first integral is given by

$$
k^{2} \int_{\Gamma_{K} \backslash \mathbb{H}^{2}} G_{p} \omega^{2}=-k^{2} \operatorname{vol}(T(p))\left(2 \frac{L^{\prime}\left(-1, \chi_{D}\right)}{L\left(-1, \chi_{D}\right)}+\frac{p-1}{p+1} \log (p)+1+\log (D)\right) .
$$

Here, we have used the fact that $\chi_{D}(p)=1$. For the remaining integral, we use the morphism $\varphi$ defined by (3.20) to infer

$$
\begin{aligned}
& \frac{1}{2 \pi i} \int_{T(p)^{\prime}} \mathfrak{g}(F) * \mathfrak{g}(G) \\
& =\frac{1}{2 \pi i} \int_{X_{0}(p)} \varphi^{*} \mathfrak{g}(F) * \varphi^{*} \mathfrak{g}(G) \\
& \quad=-k^{2} \operatorname{vol}(T(p))\left(4 \frac{\zeta^{\prime}(-1)}{\zeta(-1)}+2\right)-\left(\operatorname{div}\left(\varphi^{*} F\right), \operatorname{div}\left(\varphi^{*} G\right)\right)_{X_{0}(p), \text { fin }},
\end{aligned}
$$


where the last equality was derived by means of Theorem 3.13. Adding the above expressions, the claim follows by the identity $\zeta_{K}(s)=\zeta(s) L\left(s, \chi_{D}\right)$.

\section{Borcherds products on Hilbert modular surfaces}

It was shown in $[\mathrm{Br} 1$, Section 4$]$ that for certain integral linear combinations of the $G_{m}\left(z_{1}, z_{2}\right)$, all Fourier coefficients, whose index $v \in \mathfrak{d}^{-1}$ has negative norm, vanish. Such a linear combination is then the logarithm of the Petersson metric of a Hilbert modular form, which has a Borcherds product expansion. We now explain this in more detail.

\subsection{Basic properties of Borcherds products}

Recall our assumption that $D$ be a prime. Let $k$ be an integer. We denote by $A_{k}\left(D, \chi_{D}\right)$ the space of weakly holomorphic modular forms of weight $k$ with character $\chi_{D}$ for the group $\Gamma_{0}(D)$. These are holomorphic functions $f: \mathbb{H} \rightarrow \mathbb{C}$, which satisfy the transformation law

$$
f(M \tau)=\chi_{D}(d)(c \tau+d)^{k} f(\tau)
$$

for all $M=\left(\begin{array}{ll}a & b \\ c & d\end{array}\right) \in \Gamma_{0}(D)$, and are meromorphic at the cusps of $\Gamma_{0}(D)$. If $f=$ $\sum_{n \in \mathbb{Z}} c(n) q^{n} \in A_{k}\left(D, \chi_{D}\right)$, then the Fourier polynomial

$$
\sum_{n<0} c(n) q^{n}
$$

is called the principal part of $f$. Here, $q=e^{2 \pi i \tau}$, as usual. We write $M_{k}\left(D, \chi_{D}\right)$ (resp., $\left.S_{k}\left(D, \chi_{D}\right)\right)$ for the subspace of holomorphic modular forms (resp., cusp forms).

For $\epsilon \in\{ \pm 1\}$, we let $A_{k}^{\epsilon}\left(D, \chi_{D}\right)$ be the subspace of all $f=\sum_{n \in \mathbb{Z}} c(n) q^{n}$ in $A_{k}\left(D, \chi_{D}\right)$ for which $c(n)=0$ if $\chi_{D}(n)=-\epsilon$ (see [BB]). A classical lemma due to Hecke implies that

$$
A_{k}\left(D, \chi_{D}\right)=A_{k}^{+}\left(D, \chi_{D}\right) \oplus A_{k}^{-}\left(D, \chi_{D}\right)
$$

(see, e.g., [O, Lemma 6, p. 32]). Finally, we define the spaces $M_{k}^{\epsilon}\left(D, \chi_{D}\right)$ and $S_{k}^{\epsilon}\left(D, \chi_{D}\right)$ analogously.

Here, we mainly consider $M_{2}^{+}\left(D, \chi_{D}\right)$ and $A_{0}^{+}\left(D, \chi_{D}\right)$. The Eisenstein series

$$
E(\tau)=1+\sum_{n \geq 1} B_{D}(n) q^{n}=1+\frac{2}{L\left(-1, \chi_{D}\right)} \sum_{n \geq 1} \sigma_{n}(-1) q^{n}
$$

is a special element of $M_{2}^{+}\left(D, \chi_{D}\right)$. Note that by (3.17),

$$
B_{D}(n)=-2 \varphi_{n}(1)=-\frac{4}{\zeta_{K}(-1)} \operatorname{vol}(T(n)) .
$$


The space $M_{2}^{+}\left(D, \chi_{D}\right)$ is the orthogonal sum of $\mathbb{C} E$ and the subspace of cusp forms $S_{2}^{+}\left(D, \chi_{D}\right)$.

The existence of weakly holomorphic modular forms in $A_{0}^{+}\left(D, \chi_{D}\right)$ with the prescribed principal part is dictated by $S_{2}^{+}\left(D, \chi_{D}\right)$. Before making this more precise, it is convenient to introduce the following notation. If $\sum_{n \in \mathbb{Z}} c(n) q^{n} \in \mathbb{C}((q))$ is a formal Laurent series, we put

$$
\tilde{c}(n)= \begin{cases}2 c(n) & \text { if } n \equiv 0(\bmod D), \\ c(n) & \text { if } n \neq \equiv 0(\bmod D) .\end{cases}
$$

We now recall [BB, Theorem 6], which is a reformulation of [B2, Theorem 3.1].

\section{THEOREM 4.1}

There exists a weakly holomorphic modular form $f \in A_{0}^{+}\left(D, \chi_{D}\right)$ with the prescribed principal part $\sum_{n<0} c(n) q^{n}$ (where $c(n)=0$ if $\chi_{D}(n)=-1$ ) if and only if

$$
\sum_{n<0} \tilde{c}(n) b(-n)=0
$$

for every cusp form $g=\sum_{m>0} b(m) q^{m}$ in $S_{2}^{+}\left(D, \chi_{D}\right)$. Then the constant term $c(0)$ of $f$ is given by the coefficients of the Eisenstein series $E$ :

$$
c(0)=-\frac{1}{2} \sum_{n<0} \tilde{c}(n) B_{D}(-n)=\frac{2}{\zeta_{K}(-1)} \sum_{n<0} \tilde{c}(n) \operatorname{vol}(T(-n)) .
$$

Using orthogonality relations for the nondegenerate bilinear pairing between Fourier polynomials in $\mathbb{C}\left[q^{-1}\right]$ and formal power series in $\mathbb{C}[[q]]$ given by

$$
\left\{\sum_{n \leq 0} c(n) q^{n}, \sum_{m \geq 0} b(m) q^{m}\right\}=\sum_{n \leq 0} \tilde{c}(n) b(-n),
$$

one can deduce the following.

\section{COROLLARY 4.2}

A formal power series $\sum_{n \geq 0} b(n) q^{n} \in \mathbb{C}[[q]]$ (where $b(n)=0$ if $\chi_{D}(n)=-1$ ) is a modular form in $M_{2}^{+}\left(D, \chi_{D}\right)$ if and only if

$$
\sum_{n \leq 0} \tilde{c}(n) b(-n)=0
$$

for every $f=\sum_{n \gg-\infty} c(n) q^{n}$ in $A_{0}^{+}\left(D, \chi_{D}\right)$. 
By Borcherds's theory [B1, Theorem 13.3], there is a lift from weakly holomorphic modular forms in $A_{0}^{+}\left(D, \chi_{D}\right)$ to Hilbert modular forms for the group $\Gamma_{K}$, whose divisors are linear combinations of Hirzebruch-Zagier divisors. Since this result is vital for us, we state it in detail.

THEOREM 4.3 (see [B1, Theorem 13.3], [Br1, Theorem 5], [BB, Theorem 9]) Let $f=\sum_{n \in \mathbb{Z}} c(n) q^{n} \in A_{0}^{+}\left(D, \chi_{D}\right)$, and assume that $\tilde{c}(n) \in \mathbb{Z}$ for all $n<0$. Then there is a meromorphic function $F\left(z_{1}, z_{2}\right)$ on $\mathbb{H}^{2}$ with the following properties.

(i) $\quad F$ is a meromorphic modular form for $\Gamma_{K}$ with some multiplier system of finite order. The weight of $F$ is equal to the constant coefficient $c(0)$ of $f$. It can also be computed using Theorem 4.1 .

(ii) The divisor of $F$ is determined by the principal part of $f$. It equals

$$
\sum_{n<0} \tilde{c}(n) T(-n)
$$

(iii) Let $W \subset \mathbb{H}^{2}$ be a Weyl chamber attached to $f$, that is, a connected component of

$$
\mathbb{H}^{2}-\bigcup_{\substack{n<0 \\ c(n) \neq 0}} S(-n)
$$

and define the "Weyl vector" $\rho_{W} \in K$ for $W$ and $f$ by

$$
\rho_{W}=\frac{\varepsilon_{0}}{\operatorname{tr}\left(\varepsilon_{0}\right)} \sum_{n<0} \tilde{c}(n) \sum_{\lambda \in R(W,-n)} \lambda .
$$

The function $F$ has the Borcherds product expansion

$$
F\left(z_{1}, z_{2}\right)=\mathbf{e}\left(\rho_{W} z_{1}+\rho_{W}^{\prime} z_{2}\right) \prod_{\substack{v \in \mathfrak{d}^{-1} \\(v, W)>0}}\left(1-\mathbf{e}\left(v z_{1}+v^{\prime} z_{2}\right)\right)^{\tilde{c}\left(D v v^{\prime}\right)} .
$$

The product converges for all $\left(z_{1}, z_{2}\right)$ with $y_{1} y_{2}>|\min \{n ; c(n) \neq 0\}| / D$ outside the set of poles.

(iv) The Petersson metric of $F$ is given by

$$
-\log \|F\|_{\text {Pet }}=\sum_{n<0} \tilde{c}(n) G_{-n}\left(z_{1}, z_{2}\right) .
$$

(v) We have

$$
\begin{aligned}
& \int_{\Gamma_{K} \backslash \mathbb{H}^{2}} \log \left\|F\left(z_{1}, z_{2}\right)\right\|_{\mathrm{Pet}} \omega^{2} \\
& \quad=\sum_{n<0} \tilde{c}(n) \operatorname{vol}(T(-n))\left(2 \frac{L^{\prime}\left(-1, \chi_{D}\right)}{L\left(-1, \chi_{D}\right)}-2 \frac{\sigma_{-n}^{\prime}(-1)}{\sigma_{-n}(-1)}+1+\log (D)\right) .
\end{aligned}
$$




\section{Proof}

The statements (i), (ii), and (iii) are proved in [BB] using [B1, Theorem 13.3]. Therefore we only have to verify (iv) and (v).

By Theorem 4.1, the existence of $f \in A_{0}^{+}\left(D, \chi_{D}\right)$ implies that condition (4.5) is fulfilled for all cusp forms $g \in S_{2}^{+}\left(D, \chi_{D}\right)$. Using Poincaré series, it is easily checked that (4.5) actually holds for all $g \in S_{2}\left(D, \chi_{D}\right)$. Thus, by [Br1, Theorem 5], the right-hand side of (4.7) is equal to the logarithm of the Petersson metric of a Hilbert modular form $F^{\prime}$ with the same divisor as $F$. Hence the quotient $F^{\prime} / F$ is a Hilbert modular form without any zeros and poles on $\mathbb{H}^{2}$ and thereby constant. This shows that (4.7) holds up to an additive constant. By comparing the constant terms in the Fourier expansions of both sides, one finds that this constant equals zero. Here, the Fourier expansion of the right-hand side is given by (2.46). This proves (iv). The last assertion follows from (iv) and (3.18) in Corollary 3.9.

Notice that in [Br1], the assertions (i) and (ii) are deduced from (iv). There, however, a slightly different product expansion is obtained, which involves Fourier coefficients of weakly holomorphic Poincaré series of weight 2. Similarly, as in [Br2, Chapter 1], these can be related to the coefficients of weakly holomorphic modular forms of weight zero. In that way, a more direct proof of Theorem 4.3 can be given. Another direct proof can be obtained by completely arguing as in [Br2]. There, the Green functions $\Phi_{m}\left(z_{1}, z_{2}, s\right)$ are constructed as regularized theta lifts of nonholomorphic Hejhal-Poincaré series of weight zero. Here, for brevity, we have preferred to argue as above. Observe that (v) also follows from [Ku5, Main Theorem 2.12].

\section{Definition 4.4}

Hilbert modular forms that arise as lifts via Theorem 4.3 are called Borcherds products. A holomorphic Borcherds product is called integral if it has trivial multiplier system and integral coprime Fourier coefficients. A meromorphic Borcherds product is called integral if it is the quotient of two holomorphic integral Borcherds products.

\section{PROPOSITION 4.5}

For any Borcherds product $F$, there exists a positive integer $N$ such that $F^{N}$ is integral.

\section{Proof}

Let $f=\sum_{n \in \mathbb{Z}} c(n) q^{n} \in A_{0}^{+}\left(D, \chi_{D}\right)$, as in Theorem 4.3, be the preimage of $F$ under the Borcherds lift. It is explained in [BB, Proposition 8] that the condition $\tilde{c}(n) \in \mathbb{Z}$ for $n<0$ automatically implies that all coefficients $c(n)$ of $f$ are rational with bounded denominators. 
Thus, if $F$ is holomorphic, the Borcherds product expansion of $F$ implies that a suitable power of $F$ has integral coprime Fourier coefficients. Since the multiplier system of $F$ has finite order, we obtain the assertion in that case.

It remains to show that any meromorphic Borcherds product is the quotient of two holomorphic Borcherds products. In view of Theorem 4.3(ii), it suffices to show that there exist two weakly holomorphic modular forms $f_{j}=\sum_{n \in \mathbb{Z}} c_{j}(n) q^{n} \in A_{0}^{+}\left(D, \chi_{D}\right)$ such that $\tilde{c}_{j}(n) \in \mathbb{Z}_{\geq 0}$ for all $n<0$ (where $j=1,2$ ) and $f=f_{1}-f_{2}$. Then $F$ is the quotient of the Borcherds lifts of $f_{1}$ and $f_{2}$. We now construct such forms explicitly.

Let

$$
E_{12}^{+}(\tau)=1+\frac{2}{L\left(-11, \chi_{D}\right)} \sum_{n \geq 1} n^{5} \sigma_{n}(-11) q^{n}
$$

be the normalized Eisenstein series in $M_{12}^{+}\left(D, \chi_{D}\right)$. It follows from the functional equation and the Euler product expansion of $L\left(s, \chi_{D}\right)$ that $L\left(-11, \chi_{D}\right)>0$. Moreover, $\sigma_{n}(-11)>0$ if $\chi_{D}(n) \neq-1$, and $\sigma_{n}(-11)=0$ if $\chi_{D}(n)=-1$. Let $\Delta=q \prod_{n \geq 1}\left(1-q^{n}\right)^{24}$ be the Delta function, let $E_{4}$ be the normalized Eisenstein series of weight 4 for $\mathrm{SL}_{2}(\mathbb{Z})$, and let $j=E_{4}^{3} / \Delta$ be the $j$-function. The partition theoretic interpretation of $1 / \Delta$ shows that all Fourier coefficients of $1 / \Delta$ and $j$ with index at least -1 are positive integers. Consequently, if $c$ is a positive integer, then

$$
g(\tau)=\frac{E_{12}^{+}(\tau)}{\Delta(D \tau)} j(D \tau)^{c-1} \in A_{0}^{+}\left(D, \chi_{D}\right),
$$

and the Fourier coefficients $b(n)$ of $g$ satisfy

$$
\begin{array}{ll}
b(n)=0 & \text { if } n<-D c, \\
b(n)=0 & \text { if } n \geq-D c \text { and } \chi_{D}(n)=-1, \\
b(n)>0 & \text { if } n \geq-D c \text { and } \chi_{D}(n) \neq-1 .
\end{array}
$$

Thus, if we choose $c$ large enough, then there is a positive integer $c^{\prime}$ such that $f_{1}:=f+c^{\prime} g$ and $f_{2}:=c^{\prime} g$ are elements of $A_{0}^{+}\left(D, \chi_{D}\right)$ with the required properties.

\section{Remark 4.6}

According to a result of Hecke, the dimension of $M_{2}^{+}\left(D, \chi_{D}\right)$ is given by $[(D+19) / 24]$. In particular, $S_{2}^{+}\left(D, \chi_{D}\right)=\{0\}$ for the primes $D=5,13,17$. In this case, for any Hirzebruch-Zagier divisor $T(m)$, there exists a Borcherds product of weight $\varphi_{m}(1)$ with divisor $T(m)$ (for explicit examples and calculations, we refer to $[\mathrm{BB}])$. 


\subsection{Density of Borcherds products}

\section{Definition 4.7}

Intersection points $z \in \Gamma_{K} \backslash \mathbb{H}^{2}$ of Hirzebruch-Zagier divisors are called special points (see [G, Chapter 5.6]).

Particular examples of special points are the elliptic fixed points (if $D>5$ ).

\section{THEOREM 4.8}

If $S \subset \Gamma_{K} \backslash \mathbb{H}^{2}$ is a finite set of special points, then there exist infinitely many meromorphic Borcherds products of nonzero weight whose divisors are disjoint from $S$ and are given by linear combinations of isotropic Hirzebruch-Zagier divisors $T(p)$ with $p$ prime and coprime to $D$.

Here and in the following, by "infinitely many Borcherds products" we understand infinitely many Borcherds products whose divisors have pairwise proper intersection.

To prove this theorem, it is convenient to view $\mathrm{SL}_{2}\left(\mathcal{O}_{K}\right)$ as an orthogonal group. We briefly recall some facts on the identification of $\left(\mathrm{SL}_{2}(\mathbb{R}) \times \mathrm{SL}_{2}(\mathbb{R})\right) /\{ \pm(1,1)\}$ with the group $\mathrm{SO}^{0}(2,2) /\{ \pm 1\}$ (for more details, see [G, Chapter 5.4] and [B2, Example 5.5]).

We consider the quadratic space $V$ and the lattices $L=L\left(\mathcal{O}_{K}\right), L^{\prime}=L^{\prime}\left(\mathcal{O}_{K}\right)$ defined at the beginning of Section 2.3. The upper half-plane $\mathbb{H}^{2}$ can be identified with the Grassmannian

$$
\operatorname{Gr}(L)=\left\{v \subset L \otimes_{\mathbb{Z}} \mathbb{R} ; \operatorname{dim}(v)=2,\left.q\right|_{v}<0\right\}
$$

of 2-dimensional negative definite subspaces of $L \otimes_{\mathbb{Z}} \mathbb{R}$. The action of $\mathrm{SL}_{2}\left(\mathcal{O}_{K}\right)$ on $\mathbb{H}^{2}$ corresponds to the linear action of $\mathrm{O}(L)$ on $\operatorname{Gr}(L)$. In terms of $\operatorname{Gr}(L)$, the Hirzebruch-Zagier divisor $T(m)$ is given by

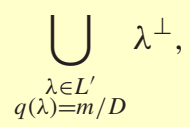

where $\lambda^{\perp}$ means the orthogonal complement of $\lambda$ in $\operatorname{Gr}(L)$.

For $v \in \operatorname{Gr}(L)$, we denote by $L_{v}$ the lattice $L^{\prime} \cap v^{\perp}$ with the integral quadratic form $q_{v}=\left.D \cdot q\right|_{L_{v}}$. If $g \in \mathrm{O}(L)$, then the quadratic modules $\left(L_{v}, q_{v}\right)$ and $\left(L_{g v}, q_{g v}\right)$ are equivalent. Therefore the equivalence classes of these quadratic forms can be viewed as invariants of the points of $\Gamma_{K} \backslash \mathbb{H}^{2}$. The following lemma is well known.

\section{LEMMA 4.9}

Let $z \in \Gamma_{K} \backslash \mathbb{H}^{2}$, and assume that $z$ corresponds to $v \in \operatorname{Gr}(L)$. Then $z \in T(m)$ if and only if the quadratic form $q_{v}$ on $L_{v}$ represents $m$. 
It is easily checked that $L_{v}$ has rank 2 if and only if $v$ corresponds to a special point $z \in \mathbb{H}^{2}$. In this case, $\left(L_{v}, q_{v}\right)$ is a positive definite integral binary quadratic form. If we write $\Delta_{v}$ for its discriminant, then $\Delta_{v}<0$ and $\Delta_{v} \equiv 0,1(\bmod 4)$.

\section{LEMMA 4.10}

If $S \subset \Gamma_{K} \backslash \mathbb{H}^{2}$ is a finite set of special points, then there are infinitely many primes $p$ coprime to $D$ such that $T(p)$ is nonempty, isotropic, and $T(p) \cap S=\emptyset$.

\section{Proof}

Let $Q_{1}, \ldots, Q_{r}$ be the positive definite integral binary quadratic forms corresponding to the special points in $S$. In view of the above discussion, it suffices to show that there exist infinitely many primes $p$, which are not represented by $Q_{1}, \ldots, Q_{r}$, and such that $\chi_{D}(p)=1$.

Let $\Delta_{j}$ be the discriminant of $Q_{j}$. Then $\Delta_{j}<0$ and $\Delta_{j} \equiv 0,1(\bmod 4)$. Let $n$ be a nonzero integer coprime to $\Delta_{j}$. It is well known that $R\left(\Delta_{j}, n\right)$, the total representation number of $n$ by positive definite integral binary quadratic forms of discriminant $\Delta_{j}$, is given by

$$
R\left(\Delta_{j}, n\right)=\sum_{d \mid n} \chi_{\Delta_{j}}(d)
$$

(see [Z2, Section 8]). Hence, if $R\left(\Delta_{j}, n\right)=0$ for $n$ coprime to $\Delta_{j}$, then $Q_{j}$ does not represent $n$. In particular, any prime $p$ with $\chi_{\Delta_{j}}(p)=-1$ is not represented by $Q_{j}$.

Thus it suffices to show that there are infinitely many primes $p$ with

$$
\begin{aligned}
\chi_{\Delta_{j}}(p) & =-1 \quad(j=1, \ldots, r), \\
\chi_{D}(p) & =1 .
\end{aligned}
$$

Since the $\Delta_{j}$ are negative and $D$ is positive, this is clearly true. In fact, even a positive proportion of primes has these properties.

For the rest of this section, we temporarily abbreviate $M:=M_{2}^{+}\left(D, \chi_{D}\right)$ and $S:=$ $S_{2}^{+}\left(D, \chi_{D}\right)$. We denote the dual $\mathbb{C}$-vector spaces by $M^{\vee}$ and $S^{\vee}$, respectively. For any positive integer $r$, the functional

$$
a_{r}: M \rightarrow \mathbb{C}, \quad f=\sum_{n} b(n) q^{n} \mapsto a_{r}(f):=b(r)
$$

is a special element of $M^{\vee}$, and $M^{\vee}$ is generated by the family $\left(a_{r}\right)_{r \in \mathbb{N}}$ as a vector space over $\mathbb{C}$. We denote by $M_{\mathbb{Z}}^{\vee}$ the $\mathbb{Z}$-submodule of $M^{\vee}$ generated by the $a_{r}(r \in \mathbb{N})$. The fact that $M$ has a basis of modular forms with integral coefficients implies that 
the rank of $M_{\mathbb{Z}}^{\vee}$ equals the dimension of $M$ and that $M_{\mathbb{Z}}^{\vee} \otimes_{\mathbb{Z}} \mathbb{C}=M^{\vee}$. We write

$$
{ }^{-}: M^{\vee} \rightarrow S^{\vee}, \quad a \mapsto \bar{a}
$$

for the natural map given by the restriction of a functional.

\section{LEMMA 4.11}

Let $I$ be an infinite set of positive integers $m$ with $\chi_{D}(m) \neq-1$, and let $A^{\vee}$ be the $\mathbb{Z}$-submodule of $M_{\mathbb{Z}}^{\vee}$ generated by the $a_{m}$ with $m \in I$. Then there is a nonzero $a \in A^{\vee}$ with the property that $\bar{a}=0$ in $S^{\vee}$.

\section{Proof}

We consider the image $\bar{A}^{\vee}$ of $A^{\vee}$ in $S^{\vee}$. It is a free $\mathbb{Z}$-module of rank $d \leq \operatorname{dim} S$. There exist $n_{1}, \ldots, n_{d} \in I$ such that $\bar{a}_{n_{1}}, \ldots, \bar{a}_{n_{d}}$ are linearly independent in $\bar{A}^{\vee}$. Then for any $m \in I$, there is a linear relation

$$
r_{0}(m) \bar{a}_{m}+r_{1}(m) \bar{a}_{n_{1}}+\cdots+r_{d}(m) \bar{a}_{n_{d}}=0
$$

in $\bar{A}^{\vee}$ with integral coefficients $r_{j}(m)$ and $r_{0}(m) \neq 0$.

If the corresponding linear combination

$$
r_{0}(m) B_{D}(m)+r_{1}(m) B_{D}\left(n_{1}\right)+\cdots+r_{d}(m) B_{D}\left(n_{d}\right)
$$

of the coefficients of the Eisenstein series $E$ does not vanish, then $a=r_{0}(m) a_{m}+$ $r_{1}(m) a_{n_{1}}+\cdots+r_{d}(m) a_{n_{d}}$ is a nonzero element of $A^{\vee}$ with the claimed property, and we are done.

We now assume that the linear combination (4.9) vanishes for all $m \in I$ and derive a contradiction. If the vector $r(m)=\left(r_{1}(m), \ldots, r_{d}(m)\right)$ is equal to zero for some $m$, then the vanishing of (4.9) implies that $r_{0}(m)=0$, contradicting our assumption on the $r_{j}(m)$. Therefore we may further assume that $r(m) \neq 0$ for all $m \in I$.

Equation (4.8) and the vanishing of (4.9) imply

$$
B_{D}(m)\left(r_{1}(m) \bar{a}_{n_{1}}+\cdots+r_{d}(m) \bar{a}_{n_{d}}\right)=\left(r_{1}(m) B_{D}\left(n_{1}\right)+\cdots+r_{d}(m) B_{D}\left(n_{d}\right)\right) \bar{a}_{m}
$$

for all $m \in I$. We write $\|r\|$ for the Euclidean norm of a vector $r=\left(r_{1}, \ldots, r_{d}\right) \in \mathbb{C}^{d}$ and also denote by $\|\cdot\|$ a norm on $S^{\vee}$, say, the operator norm. Since $\bar{a}_{n_{1}}, \ldots, \bar{a}_{n_{d}}$ are linearly independent, there exists an $\varepsilon>0$ such that

$$
\left\|r_{1} \bar{a}_{n_{1}}+\cdots+r_{d} \bar{a}_{n_{d}}\right\| \geq \varepsilon\|r\|
$$


for all $r \in \mathbb{C}^{d}$. Moreover, there exists a $C>0$ such that

$$
\left|r_{1} B_{D}\left(n_{1}\right)+\cdots+r_{d} B_{D}\left(n_{d}\right)\right| \leq C\|r\|
$$

for all $r \in \mathbb{C}^{d}$. If we insert these estimates into the norm of (4.10), we obtain

$$
\varepsilon\left|B_{D}(m)\right| \cdot\|r(m)\| \leq C\|r(m)\| \cdot\left\|\bar{a}_{m}\right\| .
$$

Since $\|r(m)\| \neq 0$, we find that

$$
\left|B_{D}(m)\right| \leq \frac{C}{\varepsilon} \cdot\left\|\bar{a}_{m}\right\|
$$

for all $m \in I$.

By (4.2), for any $\delta>0$ the coefficients $B_{D}(m)$ with $\chi_{D}(m) \neq 1$ satisfy $\left|B_{D}(m)\right| \geq$ $C^{\prime} m^{1-\delta}$ as $m \rightarrow \infty$ for some positive constant $C^{\prime}$. But the Deligne bound for the growth of the coefficients of cusp forms in $S$ implies that $\left\|\bar{a}_{m}\right\|=O_{\delta}\left(m^{1 / 2+\delta}\right)$, contradicting the above inequality.

\section{Proof of Theorem 4.8}

Let $I$ be the infinite set of primes coprime to $D$ which we get by Lemma 4.10. According to Lemma 4.11, there exists a nonzero integral finite linear combination

$$
a=\sum_{p \in I} \tilde{c}(p) a_{p} \in M_{\mathbb{Z}}^{\vee}
$$

with $\bar{a}=0$ in $S^{\vee}$. But then Theorem 4.1 implies that there is a weakly holomorphic modular form $f \in A_{0}^{+}\left(D, \chi_{D}\right)$ with the principal part $\sum_{p \in I} c(p) q^{-p}$ and nonzero constant coefficient $c(0)=-(1 / 2) \sum_{p \in I} \tilde{c}(p) B_{D}(p)$. If we apply Theorem 4.3 to this $f$, we get a Borcherds product with the claimed properties.

We may remove the primes $p$ with $c(p) \neq 0$ from the set $I$ and repeat the above construction to get another Borcherds product. By induction, we get infinitely many Borcherds products with the claimed properties.

\section{THEOREM 4.12}

Let $C \in \operatorname{Div}\left(X\left(\Gamma_{K}\right)\right)$ be a linear combination of Hirzebruch-Zagier divisors. Then there exist infinitely many meromorphic integral Borcherds products $F_{2}$ and $F_{1}$ of nonzero weights such that

(i) all possible intersections of $\operatorname{div}\left(F_{1}\right), \operatorname{div}\left(F_{2}\right)$, and $C$ are proper;

(ii) $\quad F_{2}(\kappa)=1$ at all cusps $\kappa$ of $X\left(\Gamma_{K}\right)$;

(iii) $\operatorname{div}\left(F_{1}\right)$ is a linear combination of isotropic Hirzebruch-Zagier divisors of prime discriminant $p$ coprime to $D$ (and thus $\chi_{D}(p)=1$ ). 


\section{Proof}

Since there are infinitely many anisotropic Hirzebruch-Zagier divisors, Theorem 4.1 and Lemma 4.11 imply that there are infinitely many Borcherds products $F_{2}$ of nonzero weight, whose divisor consists of anisotropic Hirzebruch-Zagier divisors, and such that $C$ and $\operatorname{div}\left(F_{2}\right)$ intersect properly. Choose such an $F_{2}$. Since $F_{2}$ has an anisotropic divisor, the Weyl vectors in the Borcherds product expansion of $F_{2}$ at the different cusps of $X\left(\Gamma_{K}\right)$ equal zero. Consequently, $F_{2}$ is holomorphic at all cusps with value 1 .

Let $S \subset \Gamma_{K} \backslash \mathbb{H}^{2}$ be the finite set of intersection points $\operatorname{div}\left(F_{2}\right) \cap C$. By Theorem 4.8, there exist infinitely many Borcherds products $F_{1}$ of nonzero weight such that $\operatorname{div}\left(F_{1}\right)$ is disjoint to $S$ and such that properties (i) and (iii) are fulfilled.

By possibly replacing $F_{2}, F_{1}$ by sufficiently large powers, we may assume that these are integral Borcherds products.

In the rest of this section, we essentially show that the subspace of $\operatorname{Pic}\left(X\left(\Gamma_{K}\right)\right) \otimes_{\mathbb{Z}} \mathbb{Q}$, spanned by all Hirzebruch-Zagier divisors, is already generated by the $T(p)$ with prime index $p$ and $\chi_{D}(p)=1$.

\section{PROPOSITION 4.13}

Let I be a set of primes containing almost all primes $p$ with $\chi_{D}(p)=1$. Then the functionals $a_{p}$ with $p \in I$ generate a $\mathbb{Z}$-submodule of finite index in $M_{\mathbb{Z}}^{\vee}$.

\section{Proof}

Since $M$ has a basis of modular forms with integral coefficients, it suffices to show that the $a_{p}$ with $p \in I$ generate $M^{\vee}$ as a $\mathbb{C}$-vector space. In view of Lemma 4.11, it suffices to show that the $\bar{a}_{p}$ generate $S^{\vee}$. Therefore the assertion is a consequence of the following lemma.

\section{LEMMA 4.14}

Let $I$ be as in Proposition 4.13. If $f \in S$ is a cusp form that is annihilated by all $\bar{a}_{p}$ with $p \in I$, then $f=0$.

\section{Proof}

Since $S$ has a basis of modular forms with rational Fourier coefficients, we may assume, without loss of generality, that the Fourier coefficients of $f$ are algebraic. By the hypothesis, and because $f \in S=S_{2}^{+}\left(D, \chi_{D}\right)$, we have $a_{p}(f)=0$ for almost all primes $p$.

The assertion follows from the properties of the $\ell$-adic Galois representations associated to a basis of normalized newforms of $S$ using the main lemma of [OS] (by a similar argument as on [OS, p. 461]). Notice that $S$ does not contain any eigenforms with complex multiplication since $D$ is a prime $\equiv 1(\bmod 4)$. 
THEOREM 4.15

Let $T(m)$ be any Hirzebruch-Zagier divisor. Then there exist infinitely many meromorphic integral Borcherds products $F$ of weight zero such that

$$
\operatorname{div}(F)=\tilde{c}(m) T(m)+\sum_{\substack{p \text { prime } \\ \chi_{D}(p)=1}} \tilde{c}(p) T(p)
$$

with suitable integral coefficients $\tilde{c}(p)$ and $\tilde{c}(m) \neq 0$.

\section{Proof}

Let $I$ be the set of all primes $p$ coprime to $m$ with $\chi_{D}(p)=1$. By Proposition 4.13, there exist integral coefficients $\tilde{c}(p)$ and a nonzero integer $\tilde{c}(m)$ such that

$$
\tilde{c}(m) a_{m}=\sum_{p \in I} \tilde{c}(p) a_{p} \in M_{\mathbb{Z}}^{\vee}
$$

Here, $\tilde{c}(p)=0$ for all but finitely many $p \in I$. Therefore, in view of Theorem 4.1, there exists a weakly holomorphic modular form $f \in A_{0}^{+}\left(D, \chi_{D}\right)$ with the principal part

$$
c(m) q^{-m}-\sum_{p \in I} c(p) q^{-p}
$$

and vanishing constant term $c(0)$. The Borcherds lift of $f$ is a Borcherds product of weight zero with divisor of the required type. By taking a sufficiently large power, we may assume that it is integral. We may now remove the primes $p$ occurring with $c(p) \neq 0$ in the above sum from the set $I$ and repeat the argument. Inductively, we find infinitely many Borcherds products of the required type.

COROLLARY 4.16

Let $T(m)$ be any Hirzebruch-Zagier divisor. Then there exist infinitely many meromorphic integral Borcherds products $F$ of nonzero weight such that

$$
\operatorname{div}(F)=\tilde{c}(m) T(m)+\sum_{\substack{p \text { prime } \\ \chi_{D}(p)=1}} \tilde{c}(p) T(p)
$$

with suitable integral coefficients $\tilde{c}(p)$ and $\tilde{c}(m) \neq 0$.

\section{Proof}

By Theorem 4.8, we can find infinitely many integral meromorphic Borcherds products $G$ of nonzero weight such that $\operatorname{div}(G)$ is a linear combination of isotropic HirzebruchZagier divisors $T(p)$ with $p$ prime and coprime to $m D$. In particular, $\operatorname{div}(G)$ does not 
have $T(m)$ as a component. The product of any such $G$ and any $F$ as in Theorem 4.15 is a Borcherds product with the required properties.

\section{Arithmetic theory of Hilbert modular surfaces}

Throughout this section, we keep the assumptions of the previous sections. In particular, $D$ is a prime congruent 1 modulo 4 and $K=\mathbb{Q}(\sqrt{D})$.

\subsection{Moduli spaces of abelian schemes with real multiplication}

In this section, we recall some background material on integral models of Hilbert modular surfaces. The reader is referred to [R], [DP], [P], and [V] for more details.

Suppose that $A \rightarrow S$ is an abelian scheme. Then there exist a dual abelian scheme $A^{\vee} \rightarrow S$ and a natural isomorphism $A \cong\left(A^{\vee}\right)^{\vee}$. If $\phi: A \rightarrow B$ is a homomorphism of abelian schemes, then there is a dual morphism $\phi^{\vee}: B^{\vee} \rightarrow A^{\vee}$. A homomorphism $\mu \in \operatorname{Hom}\left(A, A^{\vee}\right)$ is called symmetric if $\mu$ equals the composition $A \cong\left(A^{\vee}\right)^{\vee} \stackrel{\mu^{\vee}}{\longrightarrow} A^{\vee}$. In this case, we write $\mu=\mu^{\vee}$. We denote by $\operatorname{Hom}\left(A, A^{\vee}\right)^{\text {sym }}$ the space of symmetric homomorphisms.

An abelian scheme $A \rightarrow S$ of relative dimension 2, together with a ring homomorphism

$$
\iota: \mathcal{O}_{K} \longrightarrow \operatorname{End}(A)
$$

is called an abelian surface with multiplication by $\mathcal{O}_{K}$ and denoted by the pair $(A, \iota)$. Via $\alpha \mapsto \iota(\alpha)^{\vee}$, we obtain an $\mathcal{O}_{K}$-multiplication on the dual abelian surface. If $\mathfrak{a} \subset \mathcal{O}_{K}$ is an ideal, we write $A[\mathfrak{a}]$ for the $\mathfrak{a}$-torsion on $A$.

Suppose from now on that $(A, \iota)$ is an abelian surface with multiplication by $\mathcal{O}_{K}$. An element $\mu \in \operatorname{Hom}\left(A, A^{\vee}\right)$ is called $\mathcal{O}_{K}$-linear if $\mu \iota(\alpha)=\iota(\alpha)^{\vee} \mu$ for all $\alpha \in \mathcal{O}_{K}$. We denote by $P(A)$ the sheaf for the étale topology (large étale site) on $\mathrm{Sch} / S$ defined by

$$
P(A)_{T}=\left\{\lambda: A_{T} \rightarrow A_{T}^{\vee} ; \lambda \text { is symmetric and } \mathcal{O}_{K} \text {-linear }\right\}
$$

for all $T \rightarrow S$. We write $P(A)^{+}$for the subsheaf of polarizations in $P(A)$. The pair $(A, \iota)$ is said to satisfy the Deligne-Pappas condition (DP) if the canonical morphism of sheaves

$$
A \otimes_{\mathcal{O}_{K}} P(A) \longrightarrow A^{\vee}, \quad a \otimes \lambda \mapsto \lambda(a)
$$

is an isomorphism. In this case, $P(A)$ is a locally constant sheaf of projective $\mathcal{O}_{K^{-}}$ modules of rank 1 (see [V, Proposition 1.4]). The Deligne-Pappas condition (DP) is, over $\mathbb{Z}[1 / D]$-schemes, equivalent to the Rapoport condition (R) that $\Omega_{A / S}^{1}$ be locally on $S$ a free $\left(\mathcal{O}_{S} \otimes_{\mathbb{Z}} \mathcal{O}_{K}\right)$-module. Moreover, in characteristic zero, it holds automatically (see, e.g., [Go, p. 99]). 
Let $\mathfrak{l}$ be a fractional ideal of $K$, and let $\mathfrak{l}^{+}$be the subset of totally positive elements. An $\mathfrak{l}$-polarization on $(A, \iota)$ is a homomorphism of $\mathcal{O}_{K}$-modules

$$
\psi: \mathfrak{l} \longrightarrow P(A)_{S},
$$

taking $\mathfrak{l}^{+}$to $P(A)_{S}^{+}$, so that the natural homomorphism

$$
A \otimes_{\mathcal{O}_{K}} \mathfrak{l} \longrightarrow A^{\vee}, \quad a \otimes \lambda \mapsto \psi(\lambda)(a)
$$

is an isomorphism. By [V, Proposition 3.3], a homomorphism of $\mathcal{O}_{K}$-modules $\mathfrak{l} \rightarrow$ $P(A)_{S}$ is an l-polarization if and only if $(A, \iota)$ satisfies (DP) and the induced morphism of sheaves $\left(\mathfrak{l}, \mathfrak{l}^{+}\right) \rightarrow\left(P(A), P(A)^{+}\right)$for the étale topology is an isomorphism.

Let $N$ be a positive integer. Suppose that $S$ is scheme over $\operatorname{Spec} \mathbb{Z}[1 / N]$. A full level- $N$ structure on an abelian surface $A$ over $S$ with real multiplication by $\mathcal{O}_{K}$ is an $0_{K}$-linear isomorphism

$$
\left(\mathcal{O}_{K} / N \mathcal{O}_{K}\right)_{S}^{2} \longrightarrow A[N]
$$

between the constant group scheme defined by $\left(\mathcal{O}_{K} / N \mathcal{O}_{K}\right)^{2}$ and the $N$-torsion on $A$.

With the formulation of the next theorem, which summarizes some properties of the moduli spaces of abelian surfaces needed below, we follow [P, Theorem 2.1.2, p. 47; Remark 2.1.3, p. 47] (see also [C], [R], [Go]; in particular, see [Go, Theorem 2.17 , p. 57; Lemma 5.5 , p. 99]). Furthermore, we choose a primitive $N$ th root of unity $\zeta_{N}$.

\section{THEOREM 5.1}

The moduli problem "Abelian surfaces over $S$ with real multiplication by $\mathcal{O}_{K}$ and $\mathfrak{l}$-polarization with $(D P)$ and full level-N structure” is represented by a regular algebraic stack $\mathscr{H}^{\mathrm{l}}(N)$, which is flat and of relative dimension 2 over $\operatorname{Spec} \mathbb{Z}\left[\zeta_{N}, 1 / N\right]$. It is smooth over Spec $\mathbb{Z}\left[\zeta_{N}, 1 / N D\right]$, and the fiber of $\mathscr{H}^{\mathrm{l}}(N)$ above $D$ is smooth outside a closed subset of codimension 2. Moreover, if $N \geq 3$, then $\mathscr{H}(N)$ is a scheme.

It is well known that $\Gamma\left(\mathcal{O}_{K} \oplus \mathfrak{a}\right) \backslash \mathbb{H}^{2}$ can be identified with $\mathscr{H}^{\mathfrak{a d}^{-1}}(1)(\mathbb{C})$. The isomorphism can be described as follows (see, e.g., [Go, Chapter 2.2] for a detailed discussion).

To $z=\left(z_{1}, z_{2}\right) \in \mathbb{H}^{2}$, we associate the lattice

$$
\Lambda_{z}=\mathcal{O}_{K} z+\mathfrak{a}^{-1}=\left(\mathcal{O}_{K}, \mathfrak{a}^{-1}\right)\left(\begin{array}{l}
z \\
1
\end{array}\right)=\left\{\left(\begin{array}{c}
\alpha z_{1}+\beta \\
\alpha^{\prime} z_{2}+\beta^{\prime}
\end{array}\right) \in \mathbb{C}^{2} ; \alpha \in \mathcal{O}_{K}, \beta \in \mathfrak{a}^{-1}\right\}
$$


If $\gamma=\left(\begin{array}{ll}a & b \\ c & d\end{array}\right) \in \mathrm{GL}_{2}(K)$ with totally positive determinant, then

$$
\Lambda_{\gamma z}=\frac{1}{c z+d}\left(\mathcal{O}_{K}, \mathfrak{a}^{-1}\right)\left(\begin{array}{ll}
a & b \\
c & d
\end{array}\right)\left(\begin{array}{l}
z \\
1
\end{array}\right) .
$$

In particular, if $\gamma \in \Gamma\left(\mathcal{O}_{K} \oplus \mathfrak{a}\right)$, then $\Lambda_{\gamma z}=(1 /(c z+d)) \Lambda_{z}$. For any $r \in \mathfrak{a d} \mathfrak{d}^{-1}$, we define a hermitian form on $\mathbb{C}^{2}$ by

$$
H_{r, z}(u, v)=r \frac{u_{1} \bar{v}_{1}}{y_{1}}+r^{\prime} \frac{u_{2} \bar{v}_{2}}{y_{2}} \quad\left(u, v \in \mathbb{C}^{2}\right) .
$$

For $\alpha z+\beta, \gamma z+\delta \in \Lambda_{z} \subset \mathbb{C}^{2}$, we have

$$
E_{r, z}(\alpha z+\beta, \gamma z+\delta)=\Im H_{r, z}(\alpha+\beta z, \gamma+\delta z)=\operatorname{tr}(r(\alpha \delta-\beta \gamma)) \in \mathbb{Z} .
$$

The hermitian form $H_{r, z}$ is positive definite (and therefore defines a polarization) if and only if $r \in\left(\mathfrak{a d}^{-1}\right)^{+}$.

We see that $A_{z}=K \otimes_{\mathbb{Q}} \mathbb{C} / \Lambda_{z}$ is an abelian surface. On $A_{z}$, we have a natural $\mathcal{O}_{K}$-multiplication $\iota: \mathcal{O}_{K} \hookrightarrow \operatorname{End}\left(A_{z}\right)$, where $v \in \mathcal{O}_{K}$ acts via $\iota(v)=\left(\begin{array}{cc}v & 0 \\ 0 & v^{\prime}\end{array}\right)$ on $\mathbb{C}^{2}$. We write $\lambda_{r}$ for the $\mathcal{O}_{K}$-linear homomorphism in $P\left(A_{z}\right)$, given by $x \mapsto H_{r, z}(x, \cdot)$. The assignment $r \mapsto \lambda_{r}$ defines an $\mathcal{O}_{K}$-linear isomorphism $\psi: \mathfrak{a d}^{-1} \rightarrow P\left(A_{z}\right)$, which maps the totally positive elements to polarizations.

Observe that the moduli algebraic stack $\mathscr{H}^{\mathrm{l}}(N)$ depends (up to a canonical isomorphism) only on the ideal class of $\mathfrak{l}$. To lighten the notation, we frequently omit the superscript $\mathfrak{l}$ whenever $\mathfrak{l} \cong \mathfrak{d}^{-1}$; for example, we simply write $\mathscr{H}(N)$ for $\mathscr{H}^{\mathfrak{l}}(N)$. We write $\mathscr{H}=\mathscr{H}(1)$. By abuse of notation, we denote the (coarse) moduli schemes associated with $\mathscr{H}$ and $\mathscr{H}(N)$ by $\mathscr{H}$ and $\mathscr{H}(N)$, respectively.

For the following results, we refer to $[\mathrm{C}$, Theorems $3.6,4.3]$ and $[\mathrm{R}$, Theorems $5.1,6.7]$.

\section{THEOREM 5.2}

There is a toroidal compactification $h_{N}: \widetilde{\mathscr{H}}(N) \rightarrow \operatorname{Spec} \mathbb{Z}\left[\zeta_{N}, 1 / N\right]$ of $\mathscr{H}(N)$ which is smooth at infinity and such that forgetting the level induces a morphism $\pi_{N}: \widetilde{\mathscr{H}}(N) \rightarrow \widetilde{\mathscr{H}}(1)$ which is a Galois cover. The complement $\widetilde{\mathscr{H}}(N) \backslash \mathscr{H}(N)$ is a relative divisor with normal crossings.

We also refer to [C] and [R] for the fact that one can choose a compatible system of toroidal compactifications for which we have morphisms $\pi_{M, N}: \widetilde{\mathscr{H}}(M) \rightarrow \widetilde{\mathscr{H}}(N)$ whenever $N \mid M$.

THEOREM 5.3 ( $q$-expansion principle)

There is a positive integer $n_{0}$ (depending on $K$ and $N$ ) such that in all weights $k$ divisible by $n_{0}$, there exists a line bundle $\mathscr{M}_{k}\left(\Gamma_{K}(N)\right)$ on $\widetilde{\mathscr{H}}(N)$, whose global 
sections correspond to holomorphic Hilbert modular forms of weight $k$ for $\Gamma_{K}(N)$ with Fourier coefficients in $\mathbb{Z}\left[\zeta_{N}, 1 / N\right]$. If $N \geq 3$, then we can put $n_{0}=1$.

We call $\mathscr{M}_{k}\left(\Gamma_{K}(N)\right)$ the line bundle of Hilbert modular forms. It is given by the $k$ th power of the Hodge bundle, that is, the pullback along the zero section of the determinant of the relative cotangent bundle of the universal family over $\mathscr{H}(N)$.

According to Proposition 4.5, any integral Borcherds product of weight $k$ (divisible by $\left.n_{0}\right)$ defines a rational section of $\mathscr{M}_{k}\left(\Gamma_{K}(N)\right)$.

\section{THEOREM 5.4}

The minimal compactification of the (coarse) moduli scheme $\mathscr{H}(N)$ is given by

$$
\overline{\mathscr{H}}(N)=\operatorname{Proj}\left(\bigoplus_{k \geq 0, n_{0} \mid k} H^{0}\left(\widetilde{\mathscr{H}}(N), \mathscr{M}_{k}\left(\Gamma_{K}(N)\right)\right)\right) \text {. }
$$

The scheme $\overline{\mathscr{H}}(N)$ is normal, projective, and flat over $\operatorname{Spec} \mathbb{Z}\left[\zeta_{N}, 1 / N\right]$ (see [C, p. 549]). Furthermore, for $N=1$, its fibers over $\operatorname{Spec} \mathbb{Z}$ are irreducible (see [DP, p. 65]).

For any embedding $\sigma \in \operatorname{Hom}\left(\mathbb{Q}\left(\zeta_{N}\right), \mathbb{C}\right)$, the complex variety $\overline{\mathscr{H}}(N)_{\sigma}(\mathbb{C})$ is isomorphic to $X\left(\Gamma_{K}(N)\right)$ (see, e.g., [R, p. 331]).

\section{PROPOSITION 5.5}

For any $m$, the divisor $T(m) \subset X\left(\Gamma_{K}\right)=\overline{\mathscr{H}}(\mathbb{C})$ is defined over $\mathbb{Q}$.

\section{Proof}

This fact is well known (see, e.g., [HLR], [G] for related formulations). We sketch a different proof using the theory of Borcherds products.

Let $p$ be a prime that is split in $\mathcal{O}_{K}$. According to Proposition 3.10, $T(p)$ is the image of the morphism $\varphi$ defined in (3.20). By Proposition 3.11, the pullback via $\varphi$ of a Hilbert modular form with rational Fourier coefficients is a modular form for $\Gamma_{0}(p)$ with rational coefficients. Thus the ideal of holomorphic Hilbert modular forms vanishing along $T(p)$ in the graded ring of holomorphic Hilbert modular forms is generated by Hilbert modular forms with rational coefficients. Since $X\left(\Gamma_{K}\right)$ is equal to Proj $\bigoplus_{n_{0} \mid k} H^{0}\left(\widetilde{\mathscr{H}}, \mathscr{M}_{k}\left(\Gamma_{K}\right)\right)(\mathbb{C})$, we obtain the claim for $T(p)$ (see also Proposition 5.11).

If $T(m)$ is any Hirzebruch-Zagier divisor, then by Theorem 4.15, there exists an integral Borcherds product whose divisor is a linear combination of $T(m)$ and divisors $T(p)$ with prime index $p$, as above. Now the claim follows by linearity.

\section{Definition 5.6}

We define the Hirzebruch-Zagier divisor $T_{N}(m)$ on the generic fiber $\widetilde{\mathscr{H}}(N)_{\mathbb{Q}\left(\zeta_{N}\right)}$ as the pullback of $T(m)$ on $\overline{\mathscr{H}}_{\mathbb{Q}}$. Moreover, we define the Hirzebruch-Zagier divisor $\mathscr{T}_{N}(m)$ on $\widetilde{\mathscr{H}}(N)$ as the Zariski closure of $T_{N}(m)$. 
If $N=1$, we frequently write $\mathscr{T}(m)$ for $\mathscr{T}_{1}(m)$. Observe that $\mathscr{T}_{N}(m)=\pi_{N}^{*} \mathscr{T}(m)$.

\section{PROPOSITION 5.7}

Let $F$ be an integral Borcherds product of weight $k$, and write $\operatorname{div}_{N}(F)$ for the divisor on $\widetilde{\mathscr{H}}(N)$ of the corresponding global section of $\mathscr{M}_{k}\left(\Gamma_{K}(N)\right)$. Then $\operatorname{div}_{N}(F)$ is equal to the Zariski closure of the induced divisor on the generic fiber. In particular, if $\operatorname{div}(F)(\mathbb{C})=\sum_{m} a(m) T(m)$ on $X\left(\Gamma_{K}\right)$, then we have, on $\widetilde{\mathscr{H}}(N)$,

$$
\operatorname{div}_{N}(F)=\sum_{m} a(m) \mathscr{T}_{N}(m) .
$$

\section{Proof}

Without loss of generality, we may assume that $F$ is holomorphic. It suffices to show that $\operatorname{div}_{N}(F)$ is a horizontal divisor. Since $F$ is a modular form for the full group $\Gamma_{K}$, the $\operatorname{divisor}_{\operatorname{div}_{N}}(F)$ is the pullback of a divisor on $\widetilde{\mathscr{H}}$. Because $\widetilde{\mathscr{H}}$ is geometrically irreducible at all primes (see [DP, p. 65]), the vertical part of $\operatorname{div}_{N}(F)$ can contain only multiples of full fibers of $\widetilde{\mathscr{H}}(N)$. But the Borcherds product expansion implies that the Fourier coefficients of $F$ are coprime. Therefore, by the $q$-expansion principle (see [C, Theorem 4.1]), $\operatorname{div}_{N}(F)$ does not contain a full fiber of $\widetilde{\mathscr{H}}(N)$ above $\operatorname{Spec} \mathbb{Z}\left[\zeta_{N}, 1 / N\right]$. This concludes the proof of the proposition.

\subsection{Modular morphisms}

We extend the morphism $\varphi: Y_{0}(p) \rightarrow \Gamma_{K} \backslash \mathbb{H}^{2}$ of Section 3.3 to integral models by giving a modular interpretation. We basically follow the descriptions in [Go, Chapter 2.5.1] and [La, p. 134] (see also [KR, Remark (ii), p. 169] for related results). Moreover, we extend it to compactifications by means of the $q$-expansion principle.

For the rest of this section, we assume that $p$ is a prime that is split in $\mathcal{O}_{K}$ or $p=1$. Let $\mathfrak{p}$ be a prime ideal of $\mathcal{O}_{K}$ above $p$. There are a fractional ideal $\mathfrak{c}$ and a totally positive $\lambda \in K$ such that $\mathfrak{p}=\lambda \mathfrak{c}^{2}$. We may assume that $\mathrm{N}(\lambda)$ is a power of $p$ (e.g., if we take $\mathfrak{c}=\mathfrak{p}^{(h+1) / 2}$, where $h$ is the class number of $K$ ). We fix a matrix $M \in\left(\begin{array}{cc}\mathfrak{c}^{-1} & \mathfrak{c}^{-1} \\ \mathfrak{c} & \mathfrak{c}\end{array}\right) \cap \mathrm{SL}_{2}(K)$.

The following two lemmas were communicated to us by T. Wedhorn [W].

\section{LEMMA 5.8}

Let $A / S$ and $B / S$ be abelian surfaces with $\mathcal{O}_{K}$-multiplication such that $P(A)$ and $P(B)$ are locally constant sheaves of projective $\mathcal{O}_{K}$-modules of rank 1 .

(i) Let $\pi: A \rightarrow B$ be an $\mathcal{O}_{K}$-linear isogeny whose degree is invertible on $S$, and denote by $\pi^{*}: P(B) \hookrightarrow P(A), g \mapsto \pi^{\vee} g \pi$ the canonical map. Then the index of $\pi^{*} P(B)$ in $P(A)$ is equal to the degree of $\pi$.

(ii) Let $\mathfrak{a}$ be an ideal of $\mathcal{O}_{K}$, and let $\pi: A \rightarrow B:=A / A[\mathfrak{a}]$ be the canonical projection. Then $\mathfrak{a}^{2} P(A) \subset \pi^{*} P(B)$. If the norm of $\mathfrak{a}$ is invertible on $S$, we have equality. 
Proof

(i) We write ()$_{p}$ for ( $) \otimes_{\mathbb{Z}} \mathbb{Z}_{p}$. It suffices to show that the length of the cokernel of $\pi_{p}^{*}: P(B)_{p} \hookrightarrow P(A)_{p}$ is equal to the $p$-adic valuation of $\operatorname{deg}(\pi)$ for any prime number $p$ that divides the degree of $\pi$. We have a commutative diagram

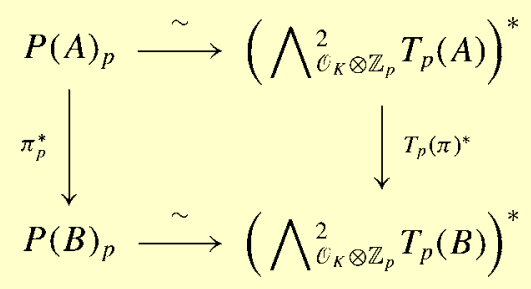

where $T_{p}(A)$ and $T_{p}(B)$ denote the Tate modules corresponding to $A$ and $B$, and for an alternating form of $\left(\mathcal{O}_{K} \otimes \mathbb{Z}_{p}\right)$-modules $\beta: \bigwedge_{\mathcal{O}_{K} \otimes \mathbb{Z}_{p}}^{2} T_{p}(A) \rightarrow \mathcal{O}_{K} \otimes \mathbb{Z}_{p}$, we put $T_{p}(\xi)^{*}(\beta)=\beta \circ \bigwedge^{2} T_{p}(\xi)$ (see, e.g., [V, (1.7.4)]). Since

$$
\begin{aligned}
v_{p}(\operatorname{deg}(\pi)) & =\operatorname{length}\left(\operatorname{Coker}\left(T_{p}(\pi)\right)\right) \\
& =\operatorname{length}\left(\operatorname{Coker}\left(T_{p}(\pi)^{*}\right)\right) \\
& =\operatorname{length}\left(\operatorname{Coker}\left(\pi_{p}^{*}\right)\right),
\end{aligned}
$$

we obtain the first claim.

(ii) Let $C$ be any abelian scheme over $S$ with $\mathcal{O}_{K}$-multiplication. We first claim that $\mathfrak{a} \operatorname{Hom}_{\mathcal{O}_{K}}(A, C) \subset \operatorname{Hom}_{\mathcal{O}_{K}}(B, C) \pi$. Let $x \in \mathfrak{a}$, and let $\alpha \in \operatorname{Hom}_{\mathcal{O}_{K}}(A, C)$. We have to show that $x \alpha$ annihilates $A[\mathfrak{a}]$, but this is obvious as $\alpha$ is $\mathcal{O}_{K}$-linear.

Next, we claim that $\mathfrak{a H o m}_{\mathcal{O}_{K}}\left(C, A^{\vee}\right) \subset \pi^{\vee} \operatorname{Hom}_{\mathcal{O}_{K}}\left(C, B^{\vee}\right)$. Note that if we dualize the canonical projection $\pi: A \rightarrow B, \pi^{\vee}$ is the canonical projection $B^{\vee} \rightarrow B^{\vee} / B^{\vee}[\mathfrak{a}]$. This follows from the fact that the dual of the multiplication with an element $x \in \mathcal{O}_{K}$ on any abelian scheme $A$ with $\mathcal{O}_{K}$-multiplication is just the multiplication with $x$ on $A^{\vee}$. Hence the second claim follows from the first one by dualizing.

Altogether, these two claims imply that $\mathfrak{a}^{2} \operatorname{Hom}_{\mathcal{O}_{K}}\left(A, A^{\vee}\right) \subset \pi^{*} \operatorname{Hom}_{\mathcal{O}_{K}}\left(B, B^{\vee}\right)$, and hence $\mathfrak{a}^{2} P(A) \subset \pi^{*} P(B)$.

It follows that $\mathfrak{a}^{2} P(A) \subset \pi^{*} P(B) \subset P(A)$, and as $P(A)$ is a locally constant sheaf of projective $\mathcal{O}_{K}$-modules of rank 1 , this is also true for $P(B)$. Moreover, the degree of $\pi$ is $N(\mathfrak{a})^{2}$. Hence the assertion follows from (i).

\section{LEMMA 5.9}

Let $S$ be a scheme over $\mathbb{Z}[1 / p]$, and let $A / S$ be an abelian surface with $\mathcal{O}_{K^{-}}$ multiplication such that $P(A)$ is a locally constant sheaf of projective $\mathcal{O}_{K}$-modules of rank 1. Let $0 \subsetneq H \subsetneq A[\mathfrak{p}]$ be an $\mathcal{O}_{K}$-invariant locally free subgroup scheme, and 
write $\pi: A \rightarrow A / H$ for the canonical projection. Then the image of the canonical map $\pi^{*}: P(A / H) \rightarrow P(A)$ is equal to $\mathfrak{p} P(A)$.

\section{Proof}

If we briefly write $B=A / H$, we have a commutative diagram

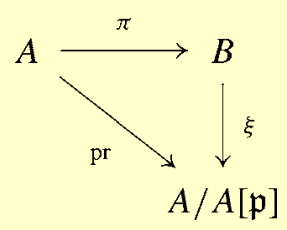

where pr denotes the natural projection. The morphism $\xi$ exists because $H \subset A[\mathfrak{p}]$. The condition $0 \subsetneq H \subsetneq A[\mathfrak{p}]$ implies that both $\pi$ and $\xi$ have degree greater than 1 . Since the degree of pr is equal to $p^{2}$, we have $\operatorname{deg}(\pi)=\operatorname{deg}(\xi)=p$. In view of Lemma 5.8, the above diagram induces the following inclusions for the polarization modules:

$$
\mathfrak{p}^{2} P(A)=\operatorname{pr}^{*} P(A / A[\mathfrak{p}]) \subsetneq \pi^{*} P(B) \subsetneq P(A) .
$$

Therefore $\pi^{*} P(B)=\mathfrak{p} P(A)$.

Let $l$ be a fractional ideal of $K$, and let $E / S$ be an elliptic curve over a scheme $S$. We consider the abelian surface

$$
E \otimes_{\mathbb{Z}} \mathfrak{l} \cong E \times_{S} E
$$

with the canonical $\mathcal{O}_{K}$-action, the isomorphism being obtained by choosing a $\mathbb{Z}$-basis to $\mathfrak{l}$. Then the natural $\mathcal{O}_{K}$-action on $\mathfrak{l}$ is given by a ring homomorphism $\mathcal{O}_{K} \hookrightarrow$ $\mathrm{M}_{2}(\mathbb{Z}), u \mapsto R_{u}$. The corresponding $\mathcal{O}_{K}$-action on $E \times_{S} E$ is given by the inclusion $\mathrm{M}_{2}(\mathbb{Z}) \subset \operatorname{End}\left(E \times{ }_{S} E\right)$. One easily checks that for any $u \in \mathcal{O}_{K}$, the conjugate acts by $R_{u^{\prime}}=R_{u}^{* t}=\left(\begin{array}{cc}0 & -1 \\ 1 & 0\end{array}\right) R_{u}\left(\begin{array}{cc}0 & 1 \\ -1 & 0\end{array}\right)$.

\section{LEMMA 5.10}

The dual of the abelian surface with $\mathcal{O}_{K}$-multiplication $E \otimes_{\mathbb{Z}} \mathfrak{l}$ is given by $E \otimes_{\mathbb{Z}}(\mathfrak{l} \mathfrak{d})^{-1}$. Moreover, $P\left(E \otimes_{\mathbb{Z}} \mathfrak{l}\right) \cong \mathfrak{l}^{-2} \mathfrak{d}^{-1}$, the isomorphism preserves the positivity, and the Deligne-Pappas condition (DP) holds.

\section{Proof}

If we choose a $\mathbb{Z}$-basis of $\mathfrak{l}$, the natural $\mathcal{O}_{K}$-action on $\mathfrak{l}$ is given by a ring homomorphism $\mathcal{O}_{K} \hookrightarrow \mathrm{M}_{2}(\mathbb{Z}), x \mapsto R_{x}$. The dual of $\mathfrak{l}$ with respect to the trace form on $K$ is equal to $(\mathfrak{l})^{-1}$, and the dual $\mathcal{O}_{K}$-action with respect to the dual basis is given by $x \mapsto R_{x}^{t}$.

On the other hand, we may identify $\left(E \times{ }_{S} E\right)^{\vee}$ canonically with $E \times{ }_{S} E$. This identifies the dual $R^{\vee}$ of a morphism $R \in \mathrm{M}_{2}(\mathbb{Z}) \subset \operatorname{End}\left(E \times{ }_{S} E\right)$ with $R^{t}$. This yields the first assertion. 
We obtain an $\mathcal{O}_{K}$-linear monomorphism $\mathfrak{l}^{-2} \mathfrak{d}^{-1} \rightarrow P\left(E \otimes_{\mathbb{Z}} \mathfrak{l}\right)$ by the assignment $x \mapsto(e \otimes l \mapsto e \otimes x l)$. One can check that totally positive elements are mapped to polarizations. The natural composite morphism

$$
\left(E \otimes_{\mathbb{Z}} \mathfrak{l}\right) \otimes_{\mathcal{O}_{K}}\left(\mathfrak{l}^{-2} \mathfrak{d}^{-1}\right) \longrightarrow\left(E \otimes_{\mathbb{Z}} \mathfrak{l}\right) \otimes_{\mathcal{O}_{K}} P\left(E \otimes_{\mathbb{Z}} \mathfrak{l}\right) \longrightarrow\left(E \otimes_{\mathbb{Z}} \mathfrak{l}\right)^{\vee}
$$

is an isomorphism. Now, the assertion follows from [V, Proposition 3.3].

\section{PROPOSITION 5.11}

There exists a morphism (defined in the proof) from the moduli stack $\mathscr{Y}_{0}(p)$ over $\mathrm{Sch} / \mathbb{Z}[1 / p]$ of elliptic curves, together with a cyclic subgroup of order $p$ to the moduli stack $\mathscr{H}$ over $\mathrm{Sch} / \mathbb{Z}[1 / p]$ of abelian schemes with $\mathcal{O}_{K}$-multiplication and $\mathfrak{d}^{-1}$ polarization with condition $(D P)$. Moreover, the associated morphism $\mathscr{Y}_{0}(p)(\mathbb{C}) \rightarrow$ $\mathscr{H}(\mathbb{C})$ is induced by the morphism of Section 3.3, $\mathbb{H} \rightarrow \mathbb{H}^{2}, \tau \mapsto M^{-1}\left(\begin{array}{ll}\lambda & 0 \\ 0 & 1\end{array}\right)(\tau, \tau)$.

\section{Proof}

Let $S$ be a scheme over $\mathbb{Z}[1 / p]$, let $E$ be an elliptic curve over $S$, and let $C$ be a finite locally free subgroup scheme of $E$ whose geometric fibers are cyclic groups of order p. We consider $A=E \otimes_{\mathbb{Z}} \mathfrak{c}$ as in (5.4) with $\mathfrak{c}$ as in the beginning of Section 5.2. The subgroup $C \otimes_{\mathbb{Z}} \mathfrak{c} \subset A$ of order $p^{2}$ is invariant under the action of $\mathcal{O}_{K}$. It is also invariant under the isomorphism $\sigma=\left(\begin{array}{cc}0 & -1 \\ 1 & 0\end{array}\right) \in \mathrm{SL}_{2}(\mathbb{Z}) \subset \operatorname{End}(A)$.

The p-torsion $A[\mathfrak{p}] \subset A$ is a subgroup of order $p^{2}$, which is also invariant under the action of $\mathcal{O}_{K}$, but $A[\mathfrak{p}]^{\sigma}=A\left[\mathfrak{p}^{\prime}\right]$. Consequently, $H=\left(C \otimes_{\mathbb{Z}} \mathfrak{c}\right) \cap A[\mathfrak{p}]$ is a subgroup of $A$ of order $p$, which is $\mathcal{O}_{K}$-invariant. The fact that the orders of $C \otimes_{\mathbb{Z}} \mathfrak{c}$ and $A[\mathfrak{p}]$ are invertible on the base implies that they are both étale over $S$. Hence $H$ is also étale over $S$. We have an exact sequence of group schemes with $\mathcal{O}_{K}$-multiplication

$$
0 \longrightarrow H \longrightarrow A \longrightarrow B \longrightarrow 0 \text {. }
$$

Since $H$ satisfies the conditions of [AG, Corollary 3.2], the abelian scheme $B$ satisfies (DP) for $P(B)$, which is equal to $\mathfrak{p c}^{-2} \mathfrak{d}^{-1} \cong \mathfrak{d}^{-1}$ by Lemmas 5.9 and 5.10. The assignment $(E, C) \mapsto B$ is functorial and defines a morphism $\mathscr{Y}_{0}(p) \rightarrow \mathscr{H}$.

We now trace this morphism on the complex points. Recall that points on $\Gamma_{0}(p) \backslash \mathbb{H}$ correspond to isomorphism classes of elliptic curves over $\mathbb{C}$ together with a subgroup of order $p$ via the assignment $\tau \mapsto=\left(E_{\tau}, C_{\tau}\right):=\left(\mathbb{C} / \Lambda_{\tau},\langle 1 / p\rangle\right)$, where $\Lambda_{\tau}=\mathbb{Z} \tau+\mathbb{Z}$, and $\langle 1 / p\rangle$ denotes the subgroup of $E_{\tau}$ generated by the point $1 / p+\Lambda_{\tau}$. The abelian surface $A$ is defined by the lattice $\Lambda_{\tau, \tau}=\mathfrak{c}(\tau, \tau)+\mathfrak{c}$. The lattice for the quotient $A /\left(C_{\tau} \otimes \mathfrak{c}\right)$ is given by $\mathfrak{c}(\tau, \tau)+(1 / p) \mathfrak{c} \subset \mathbb{C}^{2}$ and that for $A / A[\mathfrak{p}]$ is given by $\mathfrak{c p}^{-1}(\tau, \tau)+\mathfrak{c p}^{-1}$. Therefore the lattice for $B$ is equal to $\Lambda_{B}=\mathfrak{c}(\tau, \tau)+\mathfrak{c p}^{-1}$. On the other hand, the abelian surface corresponding to the point $M^{-1}\left(\begin{array}{cc}\lambda & 0 \\ 0 & 1\end{array}\right) z \in \Gamma_{K} \backslash \mathbb{H}^{2}$ is given by the lattice $\tilde{\Lambda}_{z}=\mathcal{O}_{K} M^{-1}\left(\begin{array}{ll}\lambda & 0 \\ 0 & 1\end{array}\right) z+\mathcal{O}_{K}$. If we write $M=\left(\begin{array}{ll}a & b \\ c & d\end{array}\right)$ and use 
(5.2), we see that

$$
\begin{aligned}
\tilde{\Lambda}_{z} & =\frac{1}{-c z+a}\left(\mathcal{O}_{K}, \mathcal{O}_{K}\right) M^{-1}\left(\begin{array}{ll}
\lambda & 0 \\
0 & 1
\end{array}\right)\left(\begin{array}{l}
z \\
1
\end{array}\right) \\
& =\frac{\lambda}{-c z+a}\left(\mathfrak{c},(\lambda \mathfrak{c})^{-1}\right)\left(\begin{array}{l}
z \\
1
\end{array}\right) \\
& =\frac{\lambda}{-c z+a}\left(\mathfrak{c} z+\mathfrak{c p}^{-1}\right) .
\end{aligned}
$$

Therefore $\Lambda_{B} \cong \tilde{\Lambda}_{(\tau, \tau)}$, and the associated morphism $\mathscr{Y}_{0}(p)(\mathbb{C}) \rightarrow \mathscr{H}(\mathbb{C})$ is induced by $\tau \mapsto M^{-1}\left(\begin{array}{ll}\lambda & 0 \\ 0 & 1\end{array}\right)(\tau, \tau)$.

\section{Remark 5.12}

(i) If we consider $A=E \otimes_{\mathbb{Z}} \mathcal{O}_{K}$ in the above construction instead of $A=E \otimes_{\mathbb{Z}} \mathfrak{c}$, we obtain a moduli description of the morphism of (3.19).

(ii) If we simply consider $E \mapsto A=E \otimes_{\mathbb{Z}} \mathcal{O}_{K}$ (and do not take a quotient), we obtain a moduli description of the diagonal embedding $\mathrm{SL}_{2}(\mathbb{Z}) \backslash \mathbb{H} \rightarrow \Gamma_{K}$ (see [Go, Chapter 2.5]).

By abuse of notation, we denote the coarse moduli scheme associated with $\mathscr{Y}_{0}(p)$ by $\mathscr{Y}_{0}(p)$, too. In the following proposition, we extend the morphism of moduli schemes $\mathscr{Y}_{0}(p) \rightarrow \mathscr{H}$ given by Proposition 5.11 to the minimal compactifications. Recall that the minimal compactification $\mathscr{X}_{0}(p)$ of $\mathscr{Y}_{0}(p)$ can be described analogously to (5.3) as

$$
\mathscr{X}_{0}(p)=\operatorname{Proj}\left(\bigoplus_{k \geq 0, n_{1} \mid k} H^{0}\left(\mathscr{X}_{0}(p), \mathscr{M}_{k}\left(\Gamma_{0}(p)\right)\right)\right) .
$$

Here, $n_{1}$ is a suitable positive integer (depending on $p$ ), and (for $k$ divisible by $n_{1}$ ) $\mathscr{M}_{k}\left(\Gamma_{0}(p)\right)$ denotes the line bundle of modular forms on $\mathscr{X}_{0}(p)$.

Moreover, by the $q$-expansion principle on modular curves, the global sections of the bundle $\mathscr{M}_{k}\left(\Gamma_{0}(p)\right)$ correspond to modular forms of weight $k$ for $\Gamma_{0}(p)$ whose Fourier coefficients at the cusp $\infty$ belong to $\mathbb{Z}[1 / p]$.

PROPOSITION 5.13

There exists a unique proper morphism $\bar{\varphi}: \mathscr{X}_{0}(p) \rightarrow \overline{\mathscr{H}}$ of schemes over $\mathbb{Z}[1 / p]$ such that the diagram

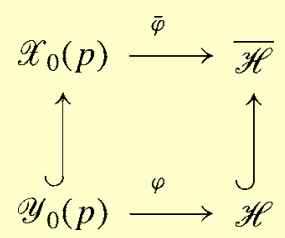


commutes. Here, $\varphi$ denotes the morphism given by Proposition 5.11. Moreover, $\bar{\varphi}$ maps the cusps of $\mathscr{X}_{0}(p)$ to cusps of $\overline{\mathscr{H}}$. The image of $\bar{\varphi}$ is the Zariski closure of the Hirzebruch-Zagier divisor $T(p)$ on the generic fiber $\overline{\mathscr{H}}_{\mathbb{Q}}$.

\section{Proof}

We have to prove the existence of $\bar{\varphi}$. The uniqueness follows from the separatedness of $\overline{\mathscr{H}}$.

We may assume that the integer $n_{1}$ of (5.5) is equal to $2 n_{0}$. The morphism $\varphi$ induces an isomorphism of line bundles on $\mathscr{Y}_{0}(p)$ :

$$
\left.\varphi^{*}\left(\mathscr{M}_{k}\left(\Gamma_{K}\right)\right) \stackrel{\sim}{\longrightarrow} \mathscr{M}_{2 k}\left(\Gamma_{0}(p)\right)\right|_{\mathscr{Y}_{0}(p)} .
$$

This can be seen by considering $\varphi$ on the universal objects over the moduli stacks $\mathscr{Y}_{0}(p)$ and $\mathscr{H}$ and using the definition of the line bundles of modular forms in terms of relative cotangent bundles. The isomorphism (5.6) induces a morphism on the sections over any open subset $U \subset \mathscr{H}$ :

$$
\varphi^{*}: \mathscr{M}_{k}\left(\Gamma_{K}\right)(U) \longrightarrow \mathscr{M}_{2 k}\left(\Gamma_{0}(p)\right)\left(\varphi^{-1} U\right), \quad s \mapsto s \circ \varphi .
$$

Consequently, $\varphi$ gives rise to a graded morphism of graded rings

$$
S:=\bigoplus_{k \geq 0, n_{0} \mid k} H^{0}\left(\widetilde{\mathscr{H}}, \mathscr{M}_{k}\left(\Gamma_{K}\right)\right) \stackrel{\varphi^{*}}{\longrightarrow} \bigoplus_{k \geq 0, n_{0} \mid k} H^{0}\left(\mathscr{Y}_{0}(p), \mathscr{M}_{2 k}\left(\Gamma_{0}(p)\right)\right) .
$$

The induced morphism over $\mathbb{C}$ is (up to a scalar power of $p$ factor) given by mapping a Hilbert modular form $F$ of weight $k$ to the modular form $\varphi^{*}(F)$ of weight $2 k$ for the group $\Gamma_{0}(p)$, as in Proposition 3.11 (which a priori may have singularities at the cusps of $\left.\Gamma_{0}(p)\right)$.

We first show that $\varphi^{*}(F) \in H^{0}\left(\mathscr{X}_{0}(p), \mathscr{M}_{2 k}\left(\Gamma_{0}(p)\right)\right)$ for any $F \in$ $H^{0}\left(\widetilde{\mathscr{H}}, \mathscr{M}_{k}\left(\Gamma_{K}\right)\right)$. This implies that $\varphi^{*}$ factors into a graded homomorphism of graded rings

$$
\bigoplus_{n_{0} \mid k} H^{0}\left(\widetilde{\mathscr{H}}, \mathscr{M}_{k}\left(\Gamma_{K}\right)\right) \stackrel{\bar{\varphi}^{*}}{\longrightarrow} \bigoplus_{n_{0} \mid k} H^{0}\left(\mathscr{X}_{0}(p), \mathscr{M}_{2 k}\left(\Gamma_{0}(p)\right)\right)
$$

and the homomorphism induced by the inclusion $\mathscr{Y}_{0}(p) \rightarrow \mathscr{X}_{0}(p)$.

Over the complex points, this follows by looking at the Fourier expansions. By Proposition 3.11(ii), we see that $\varphi^{*}(F)$ is given by (3.21) and (3.22), and therefore $\varphi^{*}(F) \in H^{0}\left(\mathscr{X}_{0}(p)(\mathbb{C}), \mathscr{M}_{2 k}\left(\Gamma_{0}(p)\right)\right)$. Moreover, Hilbert modular forms with integral Fourier coefficients are mapped to modular forms for $\Gamma_{0}(p)$ with coefficients in $\mathbb{Z}[1 / p]$. The algebraic $q$-expansion is equal to the holomorphic Fourier expansion. 
Since taking $q$-expansions commutes with base change, the map on $q$-expansions given by (3.21) and (3.22) defines the desired homomorphism $\bar{\varphi}^{*}$ over $\mathbb{Z}[1 / p]$.

By [H, Exercise 2.2.14], $\bar{\varphi}^{*}$ induces a morphism

$$
U \stackrel{\bar{\varphi}}{\longrightarrow} \operatorname{Proj} S=\overline{\mathscr{H}},
$$

defined on some open subset $U \subset \operatorname{Proj} \bigoplus_{n_{0} \mid k} H^{0}\left(\mathscr{X}_{0}(p), \mathscr{M}_{2 k}\left(\Gamma_{0}(p)\right)\right)$. By construction, $U$ contains $\mathscr{Y}_{0}(p)$. We now prove that $U=\mathscr{X}_{0}(p)$. It suffices to show that there is an $F \in S_{+}$such that $\bar{\varphi}^{*}(F)$ does not vanish on the cusps 0 and $\infty$ as sections over $\mathbb{Z}[1 / p]$. By the $q$-expansion principle, it suffices to show that there is a holomorphic Hilbert modular $F$ of positive weight with integral Fourier coefficients such that the constant coefficient of $\bar{\varphi}^{*}(F)$ at $\infty$ (resp., 0 ) is a unit in $\mathbb{Z}[1 / p]$. Since the constant coefficient of $\bar{\varphi}^{*}(F)$ at $\infty$ is up to a power of $p$ equal to the constant coefficient of $F$ at $\mathfrak{c}$ (resp., the constant coefficient of $\bar{\varphi}^{*}(F)$ at 0 is up to a power of $p$ equal to the constant coefficient of $F$ at $\mathrm{c}^{-1}$ ), it suffices to show that there is a holomorphic Hilbert modular form $F$ of positive weight with integral Fourier coefficients whose constant coefficient at $\mathfrak{c}\left(\right.$ resp., $\left.\mathfrak{c}^{-1}\right)$ is a unit in $\mathbb{Z}[1 / p]$. But the existence of such an $F$ follows from [C, Proposition 4.5]. (Alternatively, we can use an integral Borcherds product whose divisor consists of anisotropic Hirzebruch-Zagier divisors.)

The properness of $\bar{\varphi}$ follows from [H, Corollary 2.4.8(e)]. By Proposition 5.11, we know that the Hirzebruch-Zagier divisor $T(p)$ on the generic fiber $\overline{\mathscr{H}}_{\mathbb{Q}}$ is equal to the image of $\bar{\varphi} \otimes \mathbb{Q}$. Since $\bar{\varphi}$ is proper, it follows that the Zariski closure of $T(p)$ is contained in the image of $\bar{\varphi}$. The assertion follows from the irreducibility of $\mathscr{X}_{0}(p)$.

\section{Remark 5.14}

The above argument, in particular, shows that for a Hilbert modular form $F$ of weight $k$ with rational coefficients, the pullback to $\mathscr{X}_{0}(p)$ of the corresponding section of $\mathscr{M}_{k}\left(\Gamma_{K}\right)$ over $\mathbb{Z}[1 / p]$ is equal to the section of $\mathscr{M}_{2 k}\left(\Gamma_{0}(p)\right)$ over $\mathbb{Z}[1 / p]$ corresponding to the pullback $\bar{\varphi}^{*}(F)$ over $\mathbb{C}$.

\section{PROPOSITION 5.15}

Let $F, G$ be Hilbert modular forms for $\Gamma_{K}$ with rational coefficients of weight $k$ (with $k$ divisible by $n_{0}$ and $n_{1}$ ). Assume that all possible intersections among $\operatorname{div} F$, $\operatorname{div} G$, and $T(p)$ on $X\left(\Gamma_{K}\right)$ are proper. Let $\operatorname{div}_{N}(F), \operatorname{div}_{N}(G)$ be the divisors on $\widetilde{\mathscr{H}}(N)$ of the rational sections of $\mathscr{M}_{k}\left(\Gamma_{K}(N)\right)$ associated with $F$ and $G$. Moreover, let $S=\operatorname{Spec} \mathbb{Z}[1 / N p]$ and $g: \mathscr{X}_{0}(p) \rightarrow S$ be given by the structure morphism. Then we have, in $\mathrm{CH}_{\text {fin }}^{1}(S)=\mathrm{Z}^{1}(S)$,

$$
\left(h_{N}\right)_{*}\left(\mathscr{T}_{N}(p) \cdot \operatorname{div}_{N}(F) \cdot \operatorname{div}_{N}(G)\right)=\operatorname{deg}\left(\pi_{N}\right) \cdot g_{*}\left(\operatorname{div}\left(\bar{\varphi}^{*} F\right) \cdot \operatorname{div}\left(\bar{\varphi}^{*} G\right)\right) .
$$




\section{Proof}

In the following, we consider all schemes as schemes over $S$. We use the diagram

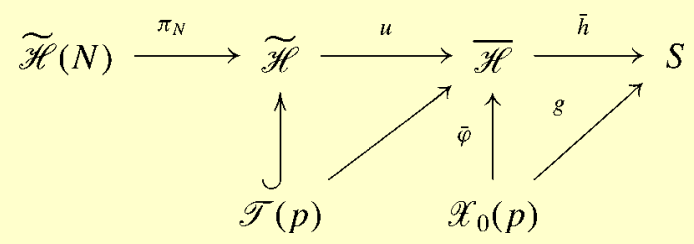

We may view $F$ and $G$ as sections of the line bundle of modular forms on $\widetilde{\mathscr{H}}(N)$, $\widetilde{\mathscr{H}}$, and $\overline{\mathscr{H}}$. Throughout the proof, we temporarily denote the corresponding Cartier divisors by $\operatorname{div}_{N}(F), \operatorname{div}_{1}(F)$, and $\operatorname{div}(F)$, respectively (and analogously for $G$ ). So, $\operatorname{div}_{N}(F)=\pi_{N}^{*} \operatorname{div}_{1}(F)$ and $\operatorname{div}_{1}(F)=u^{*} \operatorname{div}(F)$. We may use intersection theory for the intersection of a Cartier divisor and a cycle as described in [Fu, Chapters 1, 2] on the normal schemes $\widetilde{\mathscr{H}}$ and $\overline{\mathscr{H}}$ as intermediate steps (see [Fu, Chapter 20.1]).

By means of the projection formula, we obtain, in $\mathrm{CH}_{\left|\mathscr{T}_{N}(p)\right| \cap\left|\operatorname{div}_{N}(F)\right|}^{2}(\widetilde{\mathscr{H}})$,

$$
\begin{aligned}
\left(\pi_{N}\right)_{*}\left(\mathscr{T}_{N}(p) \cdot \operatorname{div}_{N}(F)\right) & =\left(\pi_{N}\right)_{*}\left(\pi_{N}^{*} \mathscr{T}(p) \cdot \pi_{N}^{*} \operatorname{div}_{1}(F)\right) \\
& =\left(\pi_{N}\right)_{*} \pi_{N}^{*} \mathscr{T}(p) \cdot \operatorname{div}_{1}(F) \\
& =\operatorname{deg}\left(\pi_{N}\right) \cdot \mathscr{T}(p) \cdot \operatorname{div}_{1}(F) .
\end{aligned}
$$

For the latter equality, we have used the fact that $\pi_{N}$ is a flat morphism.

Since the intersection of $\mathscr{T}_{N}(p), \operatorname{div}_{N}(F)$, and $\operatorname{div}_{N}(G)$ does not meet the generic fiber, we have, in $\mathrm{CH}_{\text {fin }}^{3}(\widetilde{\mathscr{H}})$,

$$
\begin{aligned}
\left(\pi_{N}\right)_{*}\left(\mathscr{T}_{N}(p) \cdot \operatorname{div}_{N}(F) \cdot \operatorname{div}_{N}(G)\right) & =\left(\pi_{N}\right)_{*}\left(\pi_{N}^{*} \mathscr{T}(p) \cdot \pi_{N}^{*} \operatorname{div}_{1}(F) \cdot \pi_{N}^{*} \operatorname{div}_{1}(G)\right) \\
& =\left(\pi_{N}\right)_{*}\left(\pi_{N}^{*} \mathscr{T}(p) \cdot \pi_{N}^{*} \operatorname{div}_{1}(F)\right) \cdot \operatorname{div}_{1}(G) \\
& =\operatorname{deg}\left(\pi_{N}\right) \cdot \mathscr{T}(p) \cdot \operatorname{div}_{1}(F) \cdot \operatorname{div}_{1}(G) .
\end{aligned}
$$

Proposition 5.13 implies that $u_{*}(\mathscr{T}(p))=\bar{\varphi}_{*}\left(\mathscr{X}_{0}(p)\right)$. Thus, by the projection formula, we get in $\mathrm{CH}_{\text {fin }}^{3}(\overline{\mathscr{H}})$ the equality

$$
\begin{aligned}
u_{*}\left(\mathscr{T}(p) \cdot \operatorname{div}_{1}(F) \cdot \operatorname{div}_{1}(G)\right) & =u_{*}(\mathscr{T}(p)) \cdot \operatorname{div}(F) \cdot \operatorname{div}(G) \\
& =\bar{\varphi}_{*}\left(\mathscr{X}_{0}(p)\right) \cdot \operatorname{div}(F) \cdot \operatorname{div}(G)
\end{aligned}
$$

Since $\bar{\varphi}$ is proper, again using the projection formula we find

$$
\bar{\varphi}_{*}\left(\mathscr{X}_{0}(p)\right) \cdot \operatorname{div}(F)=\bar{\varphi}_{*}\left(\mathscr{X}_{0}(p) \cdot \bar{\varphi}^{*} \operatorname{div}(F)\right),
$$


which implies in $\mathrm{CH}_{\text {fin }}^{3}(\overline{\mathscr{H}})$ the equality

$$
\begin{aligned}
\bar{\varphi}_{*}\left(\mathscr{X}_{0}(p)\right) \cdot \operatorname{div}(F) \cdot \operatorname{div}(G) & =\varphi_{*}\left(\mathscr{X}_{0}(p) \cdot \bar{\varphi}^{*} \operatorname{div}(F)\right) \cdot \operatorname{div}(G) \\
& =\bar{\varphi}_{*}\left(\mathscr{X}_{0}(p) \cdot \bar{\varphi}^{*} \operatorname{div}(F) \cdot \bar{\varphi}^{*} \operatorname{div}(G)\right) \\
& =\bar{\varphi}_{*}\left(\bar{\varphi}^{*} \operatorname{div}(F) \cdot \bar{\varphi}^{*} \operatorname{div}(G)\right) .
\end{aligned}
$$

Since $g_{*}=\bar{h}_{*} \circ \bar{\varphi}_{*}$, we find, in $\mathrm{CH}_{\text {fin }}^{1}(S)$,

$$
\bar{h}_{*} u_{*}\left(\mathscr{T}(p) \cdot \operatorname{div}_{1}(F) \cdot \operatorname{div}_{1}(G)\right)=g_{*}\left(\bar{\varphi}^{*} \operatorname{div}(F) \cdot \bar{\varphi}^{*} \operatorname{div}(G)\right) .
$$

Combining this with (5.7) and Remark 5.14, we obtain the assertion.

\section{Arithmetic intersection theory on Hilbert modular varieties}

Since there exists no arithmetic intersection theory for the stack $\widetilde{\mathscr{H}}(1)$, we work with the tower of schemes $\{\widetilde{\mathscr{H}}(N)\}_{N \geq 3}$ as a substitute for $\widetilde{\mathscr{H}}(1)$. We define in Section 6.3 the arithmetic Chow ring of the Hilbert modular variety without level structure to be the inverse limit of the arithmetic Chow rings associated to this tower. In this way, we obtain $\mathbb{R}$-valued arithmetic intersection numbers; even so, for every level $N$, we need only calculate the arithmetic intersection numbers up to contributions from the finite places dividing $N$.

We keep our assumption that $K$ be a real quadratic field with prime discriminant $D$. Moreover, let $N$ be an integer at least 3 . Throughout this section, we mainly work over the arithmetic ring $\mathbb{Z}\left[\zeta_{N}, 1 / N\right]$. We put $S_{N}=\operatorname{Spec} \mathbb{Z}\left[\zeta_{N}, 1 / N\right]$ and

$$
\mathbb{R}_{N}=\mathbb{R} /\left\langle\sum_{p \mid N} \mathbb{Q} \cdot \log (p)\right\rangle .
$$

Consequently, we have an arithmetic degree map

$$
\widehat{\operatorname{deg}}: \widehat{\mathrm{CH}}^{1}\left(S_{N}\right) \longrightarrow \mathbb{R}_{N} \text {. }
$$

We consider a toroidal compactification $h_{N}: \widetilde{\mathscr{H}}(N) \rightarrow S_{N}$ of the Hilbert modular variety $\mathscr{H}(N)$ for the principal congruence subgroup $\Gamma_{K}(N)$ as in Theorem 5.2. It is an arithmetic variety of dimension 2 over $\mathbb{Z}\left[\zeta_{N}, 1 / N\right]$ (see [BKK2, Definition 4.3]). If $\Sigma=\operatorname{Hom}\left(\mathbb{Q}\left(\zeta_{N}\right), \mathbb{C}\right)$ denotes the set of all embeddings from $\mathbb{Q}\left(\zeta_{N}\right)$ into $\mathbb{C}$, then the associated analytic space is

$$
\widetilde{\mathscr{H}}(N)_{\infty}=\widetilde{\mathscr{H}}(N)_{\Sigma}(\mathbb{C})=\coprod_{\sigma \in \Sigma} \widetilde{\mathscr{H}}(N)_{\sigma}(\mathbb{C}) .
$$

Analogously, we let $D_{\infty}=\coprod_{\sigma \in \Sigma}(\widetilde{\mathscr{H}}(N) \backslash \mathscr{H}(N))_{\sigma}(\mathbb{C})$. By construction, $D_{\infty}$ is a normal crossing divisor on $\widetilde{\mathscr{H}}(N)_{\infty}$ which is stable under $F_{\infty}$. We write $\mathscr{D}_{\text {pre }}$ for the 
Deligne algebra with pre-log-log forms along $D_{\infty}$. These data give rise to arithmetic Chow groups with pre-log-log forms denoted by $\widehat{\mathrm{CH}}^{*}\left(\widetilde{\mathscr{H}}(N), \mathscr{D}_{\text {pre }}\right)$.

We write

$$
d_{N}=\left[\mathbb{Q}\left(\zeta_{N}\right): \mathbb{Q}\right]\left[\Gamma_{K}: \Gamma_{K}(N)\right]
$$

for the total degree of the morphism $\widetilde{\mathscr{H}}(N) \rightarrow \widetilde{\mathscr{H}}$ of schemes over $\mathbb{Z}[1 / N]$.

From Proposition 2.5, we deduce that the line bundle of modular forms of weight $k$ on $\widetilde{\mathscr{H}}(N)$ equipped with the Petersson metric is a pre-log singular hermitian line bundle, which we denote by $\overline{\mathscr{M}}_{k}\left(\Gamma_{K}(N)\right)$. Its first arithmetic Chern class defines a class

$$
\widehat{\mathrm{c}}_{1}\left(\overline{\mathscr{M}}_{k}\left(\Gamma_{K}(N)\right)\right) \in \widehat{\mathrm{CH}}^{1}\left(\widetilde{\mathscr{H}}(N), \mathscr{D}_{\mathrm{pre}}\right)
$$

which one may represent by $\left(\operatorname{div}(F), \mathfrak{g}_{N}(F)\right)$, where $F$ is a Hilbert modular form of weight $k$ with Fourier coefficients in $\mathbb{Q}\left(\zeta_{N}\right)$ and $\mathfrak{g}_{N}(F)=\left(2 \pi i k \cdot \omega,-\log \left\|F^{\sigma}\right\|\right)_{\sigma \in \Sigma}$. The first arithmetic Chern class $\widehat{\mathrm{c}}_{1}\left(\overline{\mathscr{M}}_{k}\left(\Gamma_{K}(N)\right)\right)$ is linear in the weight $k$.

\subsection{Arithmetic Hirzebruch-Zagier divisors}

If we consider $G_{m}\left(z_{1}, z_{2}\right)$ as a Green function on $\Gamma_{K}(N) \backslash \mathbb{H}^{2}$, it immediately follows from Proposition 2.17 that

$$
\mathfrak{g}_{N}(m)=\left(-2 \partial \bar{\partial} G_{m}\left(z_{1}, z_{2}\right), G_{m}\left(z_{1}, z_{2}\right)\right)_{\sigma \in \Sigma}
$$

is a Green object for the divisor $T_{N}(m)_{\infty}$ on $\widetilde{\mathscr{H}}(N)_{\infty}$. We obtain the following arithmetic Hirzebruch-Zagier divisors:

$$
\widehat{\mathscr{T}}_{N}(m)=\left(\mathscr{T}_{N}(m), \mathfrak{g}_{N}(m)\right) \in \widehat{\mathrm{CH}}^{1}\left(\widetilde{\mathscr{H}}(N), \mathscr{D}_{\mathrm{pre}}\right) .
$$

Moreover, we define, for $k$ sufficiently divisible,

$$
\widehat{\mathrm{c}}_{1}\left(\overline{\mathscr{M}}_{1 / 2}^{\vee}\right)=-\frac{1}{2 k} \widehat{\mathrm{c}}_{1}\left(\overline{\mathscr{M}}_{k}\left(\Gamma_{K}(N)\right)\right) \in \widehat{\mathrm{CH}}^{1}\left(\widetilde{\mathscr{H}}(N), \mathscr{D}_{\mathrm{pre}}\right)_{\mathbb{Q}} .
$$

THEOREM 6.1

The subspace of $\widehat{\mathrm{CH}}^{1}\left(\widetilde{\mathscr{H}}(N), \mathscr{D}_{\text {pre }}\right)_{\mathbb{Q}}$ spanned by the $\widehat{\mathscr{T}}_{N}(m)$ has dimension $\operatorname{dim}_{\mathbb{C}} M_{2}^{+}\left(D, \chi_{D}\right)=[(D+19) / 24]$. It is already generated by $\left\{\widehat{\mathscr{T}}_{N}(p) ; p \in I\right\}$, where $I$ is any set consisting of all but finitely many primes that split in $\mathcal{O}_{K}$.

\section{Proof}

We choose for $\widehat{\mathscr{T}}_{N}(-n)$ the representatives given in (6.2). That the subspace of $\widehat{\mathrm{CH}}^{1}\left(\widetilde{\mathscr{H}}(N), \mathscr{D}_{\text {pre }}\right)_{\mathbb{Q}}$ spanned by the $\widehat{\mathscr{T}}_{N}(m)$ has dimension at most $\operatorname{dim}_{\mathbb{C}} M_{2}^{+}\left(D, \chi_{D}\right)$ 
follows by Theorem 4.1 arguing as in [B2, Lemma 4.4]. Here, one also needs Proposition 5.7 and its analytical counterpart Theorem 4.3(iv).

On the other hand, the dimension is at least $\operatorname{dim}_{\mathbb{C}} M_{2}^{+}\left(D, \chi_{D}\right)$ because of [Br1, Theorem 9], which says that any rational function on $X\left(\Gamma_{K}\right)$ whose divisor is supported on Hirzebruch-Zagier divisors is a Borcherds product.

Let $I$ be a set of primes as above, and let $T(m)$ be any Hirzebruch-Zagier divisor. By Theorem 4.15, there exist an integral Borcherds product of weight zero and thereby a rational function on $\widetilde{\mathscr{H}}(N)$ with divisor $\tilde{c}(m) T(m)+\sum_{p \in I} \tilde{c}(p) T(p)$ on $X\left(\Gamma_{K}\right)$ and $\tilde{c}(m) \neq 0$. We may conclude by Proposition 5.7 and Theorem 4.3(iv) that

$$
\widehat{\mathscr{T}}_{N}(m)=-\frac{1}{\tilde{c}(m)} \sum_{p \in I} \tilde{c}(p) \widehat{\mathscr{T}}_{N}(p) \in \widehat{\mathrm{CH}}^{1}\left(\widetilde{\mathscr{H}}(N), \mathscr{D}_{\mathrm{pre}}\right)_{\mathbb{Q}} \cdot
$$

This proves the theorem.

THEOREM 6.2

The arithmetic generating series

$$
\widehat{A}_{N}(\tau)=\widehat{\mathrm{c}}_{1}\left(\overline{\mathscr{M}}_{1 / 2}^{\vee}\right)+\sum_{m>0} \widehat{\mathscr{T}}_{N}(m) q^{m}
$$

is a modular form in $M_{2}^{+}\left(D, \chi_{D}\right)$ with values in $\widehat{\mathrm{CH}}^{1}\left(\widetilde{\mathscr{H}}(N), \mathscr{D}_{\mathrm{pre}}\right)_{\mathbb{Q}}$, that is, an element of $M_{2}^{+}\left(D, \chi_{D}\right) \otimes_{\mathbb{Q}} \widehat{C H}^{1}\left(\widetilde{\mathscr{H}}(N), \mathscr{D}_{\text {pre }}\right)_{\mathbb{Q}}$.

\section{Proof}

By Theorem 6.1 and in view of Corollary 4.2, it suffices to show that for any weakly holomorphic modular form $f=\sum_{n} c(n) q^{n}$ in $A_{0}^{+}\left(D, \chi_{D}\right)$, we have the relation

$$
\tilde{c}(0) \widehat{\mathrm{c}}_{1}\left(\overline{\mathscr{M}}_{1 / 2}^{\vee}\right)+\sum_{n<0} \tilde{c}(n) \widehat{\mathscr{T}}_{N}(-n)=0
$$

in $\widehat{\mathrm{CH}}^{1}\left(\widetilde{\mathscr{H}}(N), \mathscr{D}_{\text {pre }}\right)_{\mathbb{Q}}$. Since $A_{0}^{+}\left(D, \chi_{D}\right)$ has a basis of modular forms with rational coefficients, it suffices to check (6.4) for those $f$ with $\tilde{c}(n) \in \mathbb{Z}$ for $n<0$. Then, by Theorem 4.3, there exists a Borcherds product $F$ of weight $c(0)$ with divisor $\sum_{n<0} \tilde{c}(n) T(-n)$ on $X\left(\Gamma_{K}\right)$. We may assume that $F$ is an integral Borcherds product and therefore defines a section of $\mathscr{M}_{c(0)}\left(\Gamma_{K}(N)\right)$. If we choose for $\widehat{\mathscr{T}}_{N}(-n)$ the representatives of (6.2), we may conclude by Proposition 5.7 and Theorem 4.3(iv) that

$$
\sum_{n<0} \tilde{c}(n) \widehat{\mathscr{T}}_{N}(-n)=\sum_{n<0} \tilde{c}(n)\left(\mathscr{T}_{N}(-n), \mathfrak{g}_{N}(-n)\right)=\left(\operatorname{div}_{N}(F), \mathfrak{g}_{N}(F)\right) .
$$


By (1.12), the right-hand side of the latter equality equals $\widehat{\mathrm{c}}_{1}\left(\overline{\mathscr{M}}_{c(0)}\left(\Gamma_{K}(N)\right)\right)$. Using the linearity of the arithmetic Chow groups, we obtain (6.4) and hence the assertion.

\subsection{Arithmetic intersection numbers and Faltings heights}

Recall our convention that for an arithmetic cycle $\alpha \in \widehat{\mathrm{CH}}^{3}\left(\widetilde{\mathscr{H}}(N), \mathscr{D}_{\text {pre }}\right)_{\mathbb{Q}}$, we frequently write $\alpha$ instead of $\widehat{\operatorname{deg}}\left(\left(h_{N}\right)_{*} \alpha\right)$.

\section{LEMMA 6.3}

Let $k$ be a positive integer, and let $p$ be a prime that is split in $\mathcal{O}_{K}$ or $p=1$; then there is a $\gamma_{p, k} \in \mathbb{Q}$ such that we have, in $\mathbb{R}_{N}$,

$$
\begin{aligned}
& \widehat{\mathscr{T}}_{N}(p) \cdot \widehat{\mathrm{c}}_{1}\left(\overline{\mathscr{M}}_{k}\left(\Gamma_{K}(N)\right)\right)^{2} \\
& \quad=-k^{2} d_{N} \operatorname{vol}(T(p))\left(2 \frac{\zeta_{K}^{\prime}(-1)}{\zeta_{K}(-1)}+2 \frac{\zeta^{\prime}(-1)}{\zeta(-1)}+3+\log (D)\right)+\gamma_{p, k} \log (p) .
\end{aligned}
$$

In the proof of Theorem 6.4, we show that $\gamma_{p, k}=0$ when $(p, N)=1$.

\section{Proof}

Throughout the proof, all equalities that contain the image of the arithmetic degree map $\widehat{\operatorname{deg}}$ are equalities in $\mathbb{R}_{N}$.

Without loss of generality, we may assume $k$ sufficiently large. In order to prove the lemma, we represent $\widehat{\mathscr{T}}_{N}(p)$ by $\left(\mathscr{T}_{N}(p), \mathfrak{g}_{N}(p)\right)$. We also choose integral Borcherds products $F, G$ of nonzero weight $k$ so that $F(\kappa)=1$ at all cusps $\kappa$ and so that all possible intersections on $X\left(\Gamma_{K}\right)$ of $T(p), \operatorname{div}(F), \operatorname{div}(G)$ are proper. Such Borcherds products exist by Theorem 4.12. We take the pairs $\left(\operatorname{div}_{N}(F), \mathfrak{g}_{N}(F)\right)$ and $\left(\operatorname{div}_{N}(G), \mathfrak{g}_{N}(G)\right)$ as representatives for $\widehat{\mathrm{c}}_{1}\left(\overline{\mathscr{M}}_{k}\left(\Gamma_{K}(N)\right)\right)$, where we have put $\mathfrak{g}_{N}(F)=\left(2 \pi i k \omega,-\log \left\|F^{\sigma}\left(z_{1}, z_{2}\right)\right\|\right)_{\sigma \in \Sigma}$ and $\mathfrak{g}_{N}(G)$ analogously. With our convention on arithmetic intersection numbers, we have

$$
\begin{aligned}
\widehat{\mathscr{T}}_{N}(p) \cdot \widehat{\mathrm{c}}_{1}\left(\overline{\mathscr{M}}_{k}\left(\Gamma_{K}(N)\right)\right)^{2} \\
=\widehat{\operatorname{deg}}\left(\left(h_{N}\right)_{*}\left(\mathscr{T}_{N}(p) \cdot \operatorname{div}_{N}(F) \cdot \operatorname{div}_{N}(G)\right)\right) \\
\quad+\frac{1}{(2 \pi i)^{2}} \int_{\widetilde{H}_{(N)_{\infty}}} \mathfrak{g}_{N}(p) * \mathfrak{g}_{N}(F) * \mathfrak{g}_{N}(G) .
\end{aligned}
$$

For the first summand of (6.5), we obtain, by Proposition 5.15,

$$
\begin{aligned}
& \widehat{\operatorname{deg}}\left(\left(h_{N}\right)_{*}\left(\mathscr{T}_{N}(p) \cdot \operatorname{div}_{N}(F) \cdot \operatorname{div}_{N}(G)\right)\right) \\
& \quad=\operatorname{deg}\left(\pi_{N}\right) \cdot \widehat{\operatorname{deg}}\left(g_{*}\left(\operatorname{div}\left(\varphi^{*} F\right) \cdot \operatorname{div}\left(\varphi^{*} G\right)\right)\right)+\gamma_{p, k}^{\prime} \log (p)
\end{aligned}
$$

for some $\gamma_{p, k}^{\prime} \in \mathbb{Q}$. 
Since $F$ and $G$ are integral Borcherds products, they are invariant under $\operatorname{Aut}(\mathbb{C} / \mathbb{Q})$. Therefore, by means of Remark 3.5, we find, for the second summand of (6.5),

$$
\int_{\widetilde{\mathscr{H}}(N)_{\infty}} \mathfrak{g}_{N}(p) * \mathfrak{g}_{N}(F) * \mathfrak{g}_{N}(G)=\left[\mathbb{Q}\left(\zeta_{N}\right): \mathbb{Q}\right]\left[\Gamma_{K}: \Gamma_{K}(N)\right] \int_{\widetilde{X}\left(\Gamma_{K}\right)} \mathfrak{g}(p) * \mathfrak{g}(F) * \mathfrak{g}(G) .
$$

The latter integral was calculated in Theorem 3.14:

$$
\begin{aligned}
\frac{1}{(2 \pi i)^{2}} \int_{\widetilde{X}_{\left(\Gamma_{K}\right)}} \mathfrak{g}(p) * \mathfrak{g}(F) * \mathfrak{g}(G) \\
=-k^{2} \operatorname{vol}(T(p))\left(2 \frac{\zeta_{K}^{\prime}(-1)}{\zeta_{K}(-1)}+2 \frac{\zeta^{\prime}(-1)}{\zeta(-1)}+3+\log (D)\right) \\
\quad-k^{2} \operatorname{vol}(T(p)) \frac{p-1}{p+1} \log (p)-\left(\operatorname{div}\left(\varphi^{*} F\right), \operatorname{div}\left(\varphi^{*} G\right)\right)_{X_{0}(p), \text { fin }} .
\end{aligned}
$$

Observe that in this formula, the contribution of the finite primes is calculated with respect to a regular model of $\mathscr{X}_{0}(p)$ over $\operatorname{Spec} \mathbb{Z}$. Since $\operatorname{deg}\left(\pi_{N}\right)=\left[\Gamma_{K}: \Gamma_{K}(N)\right]$, and because

$$
\widehat{\operatorname{deg}}\left(g_{*}\left(\operatorname{div}\left(\varphi^{*} F\right) \cdot \operatorname{div}\left(\varphi^{*} G\right)\right)\right)=\left[\mathbb{Q}\left(\zeta_{N}\right): \mathbb{Q}\right]\left(\operatorname{div}\left(\varphi^{*} F\right), \operatorname{div}\left(\varphi^{*} G\right)\right)_{\mathscr{X}_{0}(p), \operatorname{fin}},
$$

we obtain the assertion.

\section{THEOREM 6.4}

Let $\overline{\mathscr{M}}_{k}\left(\Gamma_{K}(N)\right)$ be the line bundle of modular forms of weight $k$ on $\widetilde{\mathscr{H}}(N)$ equipped with the Petersson metric as in Definition 2.4. Then in $\mathbb{R}_{N}$, we have the following identities of arithmetic intersection numbers:

$$
\widehat{A}_{N}(\tau) \cdot \widehat{\mathrm{c}}_{1}\left(\overline{\mathscr{M}}_{k}\left(\Gamma_{K}(N)\right)\right)^{2}=\frac{k^{2}}{2} d_{N} \zeta_{K}(-1)\left(\frac{\zeta_{K}^{\prime}(-1)}{\zeta_{K}(-1)}+\frac{\zeta^{\prime}(-1)}{\zeta(-1)}+\frac{3}{2}+\frac{1}{2} \log (D)\right) \cdot E(\tau) .
$$

Here, $\widehat{A}_{N}(\tau)$ is the arithmetic generating series (6.3), and $E(\tau)$ is the holomorphic Eisenstein series of weight 2 (see (4.2)). In particular, for the arithmetic self-intersection number of $\overline{\mathscr{M}}_{k}\left(\Gamma_{K}(N)\right)$, we have, in $\mathbb{R}_{N}$,

$$
\overline{\mathscr{M}}_{k}\left(\Gamma_{K}(N)\right)^{3}=-k^{3} d_{N} \zeta_{K}(-1)\left(\frac{\zeta_{K}^{\prime}(-1)}{\zeta_{K}(-1)}+\frac{\zeta^{\prime}(-1)}{\zeta(-1)}+\frac{3}{2}+\frac{1}{2} \log (D)\right) .
$$

\section{Proof}

Throughout the proof, all equalities that contain the image of the arithmetic degree map $\widehat{\operatorname{deg}}$ are equalities in $\mathbb{R}_{N}$. 
Let us start by proving (6.6). By definition, we have

$$
\overline{\mathscr{M}}_{k}\left(\Gamma_{K}(N)\right)^{3}=\widehat{\mathrm{c}}_{1}\left(\overline{\mathscr{M}}_{k}\left(\Gamma_{K}(N)\right)\right)^{3}=\widehat{\operatorname{deg}}\left(\left(h_{N}\right)_{*}\left(\widehat{\mathrm{c}}_{1}\left(\overline{\mathscr{M}}_{k}\left(\Gamma_{K}(N)\right)\right)^{3}\right)\right) .
$$

By the multilinearity of the intersection product, we may assume $k$ to be sufficiently large. In view of Corollary 4.16, there exists a Borcherds product $F$ of positive weight $k$ with $\operatorname{divisor} \operatorname{div}(F)=\sum_{p} a(p) T(p)$, where $a(p) \neq 0$ only for primes $p$ with $\chi_{D}(p)=1$. (Apply Corollary 4.16 with $m$ equal to any given prime $p_{0}$ with $\chi_{D}\left(p_{0}\right)=1$.) We have shown in the proof of Theorem 6.2 that any such Borcherds product gives rise to a decomposition

$$
\widehat{\mathrm{c}}_{1}\left(\overline{\mathscr{M}}_{k}\left(\Gamma_{K}(N)\right)\right)=\sum_{p} a(p) \widehat{\mathscr{T}}_{N}(p) .
$$

Hence, in view of Lemma 6.3, we obtain the equality

$$
\begin{aligned}
\widehat{\mathrm{c}}_{1} & \left(\overline{\mathscr{M}}_{k}\left(\Gamma_{K}(N)\right)\right)^{3} \\
= & -k^{2} d_{N} \sum_{p} a(p) \operatorname{vol}(T(p))\left(2 \frac{\zeta_{K}^{\prime}(-1)}{\zeta_{K}(-1)}+2 \frac{\zeta^{\prime}(-1)}{\zeta(-1)}+3+\log (D)\right) \\
& +\sum_{\substack{p \\
a(p) \neq 0}} \gamma_{p, k} \log (p)
\end{aligned}
$$

for some $\gamma_{p, k} \in \mathbb{Q}$. There also exists a Borcherds product $G$ with $\operatorname{divisor} \operatorname{div}(G)=$ $\sum_{p} b(p) T(p)$, where $b(p) \neq 0$ only for primes $p$ with $\chi_{D}(p)=1$ and $a(p)=0$. By means of the well-definedness of the arithmetic intersection numbers, we find

$$
\sum_{\substack{p \text { prime } \\ a(p) \neq 0}} \gamma_{p, k} \log (p)=\sum_{\substack{p \text { prime } \\ b(p) \neq 0}} \gamma_{p, k} \log (p) \in \mathbb{R}_{N},
$$

which by the unique factorization in $\mathbb{Z}$, in turn, implies that all $\gamma_{p, k}$ vanish. This shows that the formula of Lemma 6.3 actually holds with $\gamma_{p, k}=0$. Since $F$ is a Borcherds product of weight $k$, we have the relation

$$
\frac{1}{2} \zeta_{K}(-1) k=\sum_{p} a(p) \operatorname{vol}(T(p)) .
$$

This concludes the proof of (6.6).

Now, let $T(m)$ be any Hirzebruch-Zagier divisor. In view of Corollary 4.16, there exists a Borcherds product $F$ whose divisor is equal to $a(m) T(m)+\sum_{p} a(p) T(p)$, where $a(m) \neq 0$, the $a(p)$ are integral coefficients, and $a(p) \neq 0$ only for primes $p$ 
with $\chi_{D}(p)=1$. Therefore

$$
\begin{aligned}
& \widehat{\mathscr{T}}_{N}(m) \cdot \widehat{\mathrm{c}}_{1}\left(\overline{\mathscr{M}}_{k}\left(\Gamma_{K}(N)\right)\right)^{2} \\
& =\frac{1}{a(m)}\left(\widehat{\mathrm{c}}_{1}\left(\overline{\mathscr{M}}_{k}\left(\Gamma_{K}(N)\right)\right)^{3}-\sum_{p} a(p) \widehat{\mathscr{T}}_{N}(p) \cdot \widehat{\mathrm{c}}_{1}\left(\overline{\mathscr{M}}_{k}\left(\Gamma_{K}(N)\right)\right)^{2}\right) \\
& \quad=-k^{2} d_{N} \operatorname{vol}(T(m))\left(2 \frac{\zeta_{K}^{\prime}(-1)}{\zeta_{K}(-1)}+2 \frac{\zeta^{\prime}(-1)}{\zeta(-1)}+3+\log (D)\right) .
\end{aligned}
$$

In the last equality, we have used the relation $a(m) \operatorname{vol}(T(m))=\left(\zeta_{K}(-1) / 2\right) k-$ $\sum_{p} a(p) \operatorname{vol}(T(p))$. Hence the claim follows from (4.3).

\section{THEOREM 6.5}

If $T(m)$ is an anisotropic Hirzebruch-Zagier divisor, then the Faltings height in $\mathbb{R}_{N}$ of its model $\mathscr{T}_{N}(m) \in \mathrm{Z}_{U}^{1}(\widetilde{\mathscr{H}}(N))$ is given by

$$
\mathrm{ht}_{\overline{\mathscr{M}}_{k}\left(\Gamma_{K}(N)\right)}\left(\mathscr{T}_{N}(m)\right)=-(2 k)^{2} d_{N} \operatorname{vol}(T(m))\left(\frac{\zeta^{\prime}(-1)}{\zeta(-1)}+\frac{1}{2}+\frac{1}{2} \frac{\sigma_{m}^{\prime}(-1)}{\sigma_{m}(-1)}\right) .
$$

\section{Proof}

By means of formula (1.16), the Faltings height equals

$$
\begin{aligned}
& \mathrm{ht}_{\overline{\mathscr{M}}_{k}\left(\Gamma_{K}(N)\right)}\left(\mathscr{T}_{N}(m)\right) \\
& \quad=\widehat{\mathscr{T}}_{N}(m) \cdot \widehat{\mathrm{c}}_{1}\left(\overline{\mathscr{M}}_{k}\left(\Gamma_{K}(N)\right)\right)^{2}-\sum_{\sigma \in \Sigma} \frac{1}{(2 \pi i)^{2}} \int_{\widetilde{\mathscr{H}}_{(N)_{\sigma}}} G_{m} \wedge \mathrm{c}_{1}\left(\overline{\mathscr{M}}_{k}\left(\Gamma_{K}(N)\right)\right)^{2} .
\end{aligned}
$$

The first term is computed in Theorem 6.4. The integral is, by functoriality of the intersection product, equal to $d_{N}$ times the quantity from (3.18) of Corollary 3.9.

\section{Remark 6.6}

(i) Observe that in view of Lemma 3.1, we may calculate formula (6.7) for all $T(\mathrm{~m})$ and, in particular, also for isotropic Hirzebruch-Zagier divisors. We may take this quantity as an ad hoc definition for the Faltings height of $\mathscr{T}_{N}(m)$ with respect to $\overline{\mathscr{M}}_{k}\left(\Gamma_{K}(N)\right)$. For example, if $m$ is square free and $\chi_{D}(p)=1$ for all primes $p$ dividing $m$, then up to boundary components, the normalization of $T_{N}(m)$ is isomorphic to some modular curve (see Remark 2.16). Although $\mathscr{T}_{N}(m) \notin \mathrm{Z}_{U}^{1}(\widetilde{\mathscr{H}}(N))$, we obtain, in $\mathbb{R}_{N}$,

$$
\mathrm{ht}_{\overline{\mathscr{M}}_{k}\left(\Gamma_{K}(N)\right)}\left(\mathscr{T}_{N}(m)\right)=-(2 k)^{2} \operatorname{vol}\left(T_{N}(m)\right)\left(\frac{\zeta^{\prime}(-1)}{\zeta(-1)}+\frac{1}{2}-\frac{1}{4} \sum_{p \mid m} \frac{p-1}{p+1} \log (p)\right) .
$$

Up to the sum over primes dividing the level $m$, this equals the arithmetic selfintersection number of the line bundle of modular forms on that modular curve as 
computed in [Kü2]. It would be interesting to obtain a geometric interpretation of the difference using the change of moduli problems over $\mathbb{Z}$ extending Proposition 5.11. At the primes dividing $m$, the morphism of line bundles (5.6) is not necessarily an isomorphism anymore.

(ii) Recall that if $T(m) \subset X\left(\Gamma_{K}\right)$ is anisotropic, then its normalization is isomorphic to some Shimura curve of discriminant $m$. For example, if $m$ is square free with an even number of prime factors, and $\chi_{D}(p)=-1$ for all primes $p$ dividing $m$, then $T(m)$ is anisotropic and our formula gives, in $\mathbb{R}_{N}$,

$$
\mathrm{ht}_{\overline{\mathscr{M}}_{k}\left(\Gamma_{K}(N)\right)}\left(\mathscr{T}_{N}(m)\right)=-(2 k)^{2} \operatorname{vol}\left(T_{N}(m)\right)\left(\frac{\zeta^{\prime}(-1)}{\zeta(-1)}+\frac{1}{2}-\frac{1}{4} \sum_{p \mid m} \frac{p+1}{p-1} \log (p)\right) .
$$

Related formulas have been obtained by Maillot and Roessler (see [MR1, Proposition 2.3]) using completely different techniques.

In recent work, Kudla, Rapoport, and Yang stated a conjecture for the arithmetic self-intersection number of the Hodge bundle on a Shimura curve (see [KRY, (0.17), p. 891]). In the forthcoming work [KK], it will be shown that (6.8), together with versions of Propositions 3.11 and 5.11 for Shimura curves, can be used to obtain a proof of their conjecture.

\section{3. $\mathbb{R}$-valued arithmetic intersection theory}

We now choose a family of toroidal compactifications $\widetilde{\mathscr{H}}(N), N \geq 3$, provided with a system of morphisms $\pi_{M, N}: \widetilde{\mathscr{H}}(M) \rightarrow \widetilde{\mathscr{H}}(N)$ given for all $N \mid M$ such that $\pi_{M, N} \circ \pi_{L, M}=\pi_{L, N}$.

Then we define the arithmetic Chow groups

$$
\widehat{\mathrm{CH}}^{*}\left(\widetilde{\mathscr{H}}, \mathscr{D}_{\text {pre }}\right)=\lim _{N \geq 3} \widehat{\mathrm{CH}}^{*}\left(\widetilde{\mathscr{H}}(N), \mathscr{D}_{\text {pre }}\right)
$$

The group $\widehat{\mathrm{CH}}^{*}\left(\widetilde{\mathscr{H}}, \mathscr{D}_{\text {pre }}\right)$ has a commutative and associative product defined componentwise.

An element $x \in \widehat{\mathrm{CH}}^{*}\left(\widetilde{\mathscr{H}}, \mathscr{D}_{\text {pre }}\right)$ is a family $\left(x_{N}\right)_{N \geq 3}$ with $x_{N} \in \widehat{\mathrm{CH}}^{*}\left(\widetilde{\mathscr{H}}(N), \mathscr{D}_{\text {pre }}\right)$ such that for all $N \mid M$, we have $\pi_{M, N}^{*} x_{N}=x_{M}$. Therefore

$$
\widehat{\mathscr{T}}(m):=\left(\widehat{\mathscr{T}}_{N}(m)\right)_{N \geq 3} \in \widehat{\mathrm{CH}}^{1}\left(\widetilde{\mathscr{H}}, \mathscr{D}_{\mathrm{pre}}\right) .
$$

Analogously, we define the arithmetic characteristic class $\widehat{\mathrm{c}}_{1}\left(\overline{\mathscr{M}}_{k}\right)$ of the line bundle of modular forms and the generating series $\widehat{A}(\tau)$. 


\section{PROPOSITION 6.7}

There exists a well-defined arithmetic degree map

$$
\widehat{\operatorname{deg}}: \widehat{\mathrm{CH}}^{3}\left(\widetilde{\mathscr{H}}, \mathscr{D}_{\text {pre }}\right) \rightarrow \mathbb{R} .
$$

\section{Proof}

The arithmetic degree maps $\widehat{\operatorname{deg}}: \widehat{\mathrm{CH}}^{3}\left(\widetilde{\mathscr{H}}(N), \mathscr{D}_{\text {pre }}\right) \rightarrow \mathbb{R}_{N}$ induce an arithmetic degree map

$$
\widehat{\operatorname{deg}}: \widehat{\mathrm{CH}}^{3}\left(\widetilde{\mathscr{H}}, \mathscr{D}_{\text {pre }}\right) \rightarrow \lim _{N \geq 3} \mathbb{R}_{N}
$$

given in each stage by $\widehat{\operatorname{deg}}(x)_{N}=\left(1 / d_{N}\right) \widehat{\operatorname{deg}}\left(x_{N}\right)$. But now, for any two integers $N, M \geq 3$ with $(N, M)=1$, by the unique factorization in $\mathbb{Z}$, the sequence

$$
0 \longrightarrow \mathbb{R} \longrightarrow \mathbb{R}_{N} \oplus \mathbb{R}_{M} \longrightarrow \mathbb{R}_{N M},
$$

where the first map is the diagonal and the second is the difference, is exact. Thus we have an isomorphism $\lim _{N \geq 3} \mathbb{R}_{N} \cong \mathbb{R}$.

By abuse of notation, given an element $x \in \widehat{\mathrm{CH}}^{3}\left(\widetilde{\mathscr{H}}, \mathscr{D}_{\text {pre }}\right)$, we denote also by $x$ its image $\widehat{\operatorname{deg}}(x) \in \mathbb{R}$ under the arithmetic degree map.

Then Theorem 6.4 implies the following.

\section{THEOREM 6.8}

We have the following identity of modular forms:

$$
\widehat{A}(\tau) \cdot \widehat{\mathrm{c}}_{1}\left(\overline{\mathscr{M}}_{k}\right)^{2}=\frac{k^{2}}{2} \zeta_{K}(-1)\left(\frac{\zeta_{K}^{\prime}(-1)}{\zeta_{K}(-1)}+\frac{\zeta^{\prime}(-1)}{\zeta(-1)}+\frac{3}{2}+\frac{1}{2} \log (D)\right) \cdot E(\tau) .
$$

Analogously, we can define a height pairing

$$
(\cdot \mid \cdot): \widehat{\mathrm{CH}}^{2}\left(\widetilde{\mathscr{H}}, \mathscr{D}_{\text {pre }}\right) \otimes \lim _{N \geq 3} \mathrm{Z}_{U}^{1}(\widetilde{\mathscr{H}}(N)) \longrightarrow \mathbb{R}
$$

and the associated Faltings height $\mathrm{ht} \overline{\bar{M}}_{k}(Z)=\left(\widehat{\mathrm{c}}_{1}\left(\overline{\mathscr{M}}_{k}\right)^{2} \mid Z\right)$. The sequence $\mathscr{T}(m)=\left(\mathscr{T}_{N}(m)\right)_{N \geq 3}$ belongs to $\lim _{N \geq 3} \mathrm{Z}_{U}^{1}(\widetilde{\mathscr{H}}(N))$. Theorem 6.5 now implies the following. 
THEOREM 6.9

If $T(m)$ is an anisotropic Hirzebruch-Zagier divisor, then the Faltings height of $\mathscr{T}(m)$ is given by

$$
\mathrm{ht} \overline{\mathscr{M}}_{k}(\mathscr{T}(m))=-(2 k)^{2} \operatorname{vol}(T(m))\left(\frac{\zeta^{\prime}(-1)}{\zeta(-1)}+\frac{1}{2}+\frac{1}{2} \frac{\sigma_{m}^{\prime}(-1)}{\sigma_{m}(-1)}\right) .
$$

\section{Remark 6.10}

Although it is more canonical to work with the whole tower $\{\widetilde{\mathscr{H}}(N)\}_{N \geq 3}$, the proof of Proposition 6.7 shows that it is already enough to pick up two integers $N, M \geq 3$ with $(N, M)=1$ and define arithmetic Chow groups $\widehat{\mathrm{CH}}^{*}\left(\widetilde{\mathscr{H}}_{N, M}, \mathscr{D}_{\text {pre }}\right)$ as the kernel of the morphism

$$
\left.\widehat{\mathrm{CH}}^{*}\left(\widetilde{\mathscr{H}}(N), \mathscr{Q}_{\mathrm{pre}}\right) \oplus \widehat{\mathrm{CH}}^{*}\left(\widetilde{\mathscr{H}}(M), \mathscr{Q}_{\mathrm{pre}}\right) \stackrel{\pi_{N M . N}^{*}-\pi_{N M M}^{*}}{\longrightarrow} \widehat{\mathrm{CH}} * \widetilde{\mathscr{H}}(N M), \mathscr{D}_{\mathrm{pre}}\right)
$$

Again, this group $\widehat{\mathrm{CH}}^{*}\left(\widetilde{\mathscr{H}}_{N, M}, \mathscr{D}_{\text {pre }}\right)_{\mathbb{Q}}$ has a commutative and associative product defined componentwise and an $\mathbb{R}$-valued arithmetic degree map. Similarly, we can define the arithmetic Hirzebruch-Zagier divisors, the arithmetic first Chern class of the line bundles of modular forms, and the arithmetic generating series in this group.

\section{Remark 6.11}

Finally, we note that if there were an arithmetic Chow ring for the stack $\widetilde{\mathscr{H}}(1)$ satisfying the usual functorialities, then it would map to $\widehat{\mathrm{CH}}^{*}\left(\widetilde{\mathscr{H}}, \mathscr{D}_{\text {pre }}\right)$, and the arithmetic degrees, as well as the values of the height pairing, would coincide.

One can also follow different strategies to obtain $\mathbb{R}$-valued arithmetic intersection numbers. First, we note that the proofs of Theorems 6.8 and 6.9 would directly carry over to other level structures. For instance, Pappas constructed regular models over $\mathbb{Z}$ of Hilbert modular surfaces associated to congruence subgroups $\Gamma_{00}(\mathscr{A}) \subset \Gamma_{K}$ (see $\left.[\mathrm{P}]\right)$. If there exists a toroidal compactification over $\mathbb{Z}$ of $\mathscr{H}_{00}^{\mathrm{Fil}}(\mathscr{A})($ see $[\mathrm{P}$, p. 51$])$, one can work on that arithmetic variety and derive formulas as in Theorems B and C. Alternatively, one can try to work directly on the coarse moduli space. This requires arithmetic Chow groups for singular arithmetic varieties of some kind (e.g., for arithmetic Baily-Borel compactifications of Hilbert modular surfaces), equipped with arithmetic Chern-class operations for hermitian line bundles.

Acknowledgments. While working on this article, we benefited from encouraging and stimulating discussions with many colleagues. We thank them all. In particular, we thank E. Freitag, E. Goren, W. Gubler, J. Kramer, S. Kudla, K. Ono, G. Pappas, and T. Wedhorn for their help. Finally, we thank the referees for their detailed comments, which improved the content of this article. 


\section{References}

[AS] M. ABRAMOWITZ and I. A. STEGUN, eds., Pocketbook of Mathematical Functions, Harri Deutsch, Thun, Switzerland, 1984. MR 076893130

[AG] F. ANDREATTA and E. Z. GOREN, Geometry of Hilbert modular varieties over totally ramified primes, Int. Math. Res. Not. 2003, no. 33, 1786-1835. MR 1987504 70

[B1] R. E. BORCHERDS, Automorphic forms with singularities on Grassmannians, Invent. Math. 132 (1998), 491-562. MR 1625724 3, 33, 54, 55

[B2] - The Gross-Kohnen-Zagier theorem in higher dimensions, Duke Math. J. 97 (1999), 219-233. MR 1682249 2, 3, 6, 53, 57, 77

[BGS] J.-B. BOST, H. GILLET, and C. SOULÉ, Heights of projective varieties and positive Green forms, J. Amer. Math. Soc. 7 (1994), 903-1027. MR 126010621

[Br1] J. H. BRUINIER, Borcherds products and Chern classes of Hirzebruch-Zagier divisors, Invent. Math. 138 (1999), 51 - 83. MR 1714336 3, 4, 5, 30, 31, 34, 35, 36, 37, $38,52,54,55,77$

[Br2] - Borcherds Products on $\mathrm{O}(2, l)$ and Chern Classes of Heegner Divisors, Lecture Notes in Math. 1780, Springer, Berlin, 2002. MR 1903920 3, 55

[Br3] - Two applications of the curve lemma for orthogonal groups, Math. Nachr. 274/275 (2004), 19-31. MR 20923227

[BB] J. H. BRUINIER and M. BUNDSCHUH, On Borcherds products associated with lattices of prime discriminant, Ramanujan J. 7 (2003), 49-61. MR 2035791 52, 53, $54,55,56$

[BF] J. H. BRUINIER and E. FREITAG, Local Borcherds products, Ann. Inst. Fourier (Grenoble) 51 (2001), 1-26. MR 182106536

[BFu] J. H. BRUINIER and J. FUNKE, On two geometric theta lifts, Duke Math. J. 125 (2004), 45-90. MR 209735738

[BK] J. H. BRUINIER and U. KÜHN, Integrals of automorphic Green's functions associated to Heegner divisors, Int. Math. Res. Not. 2003, no. 31, 1687-1729. MR 1981482 7

[Bu] J. I. BURGOS [GIL], Arithmetic Chow rings and Deligne-Beilinson cohomology, J. Algebraic Geom. 6 (1997), 335-377. MR 148911914

[BKK1] J. I. BURGOS GIL, J. KRAMER, and U. KÜHN, Arithmetic characteristic classes of automorphic vector bundles, Doc. Math. 10 (2005), 619-716. MR 22184022 , 7

[BKK2] - Cohomological arithmetic Chow rings, J. Inst. Math. Jussieu 6 (2007), 1-172. MR 2285241 2, 3, 4, 7, 9, 11, 12, 13, 14, 16, 17, 18, 19, 20, 21, 75

[C] C.-L. CHAI, Arithmetic minimal compactification of the Hilbert-Blumenthal moduli spaces, Ann. of Math. (2) 131 (1990), 541-554. MR 1053489 3, 27, 64, 65, 67,73

[DP] P. DELIGNE and G. PAPPAS, Singularités des espaces de modules de Hilbert, en les caractéristiques divisant le discriminant, Compositio Math. 90 (1994), 59-79. MR 1266495 3, 63, 66, 67 
[DR] P. DELIGNE and M. RAPOPORT, "Les schémas de modules de courbes elliptiques" in Modular Functions of One Variable, II (Antwerp, Belgium, 1972), Lecture Notes in Math. 349, Springer, Berlin, 1973, 143-316. MR 0337993

[F] H.-G. FRANKE, Kurven in Hilbertschen Modulfächen und Humbertschen Flächen im Siegel-Raum, Bonner Math. Schriften 104, Univ. Bonn, Mathematisches Institut, Bonn, 1977. MR 05168102

[Fr1] E. FREITAG, Eine Bemerkung zur Theorie der Hilbertschen Modulmannigfaltigkeiten hoher Stufe, Math. Z. 171 (1980), 27-35. MR 056648124

[Fr2] , Siegelsche Modulfunktionen, Grundlehren Math. Wiss. 254, Springer, Berlin, 1983. MR 087106725

[Fr3] - Hilbert Modular Forms, Springer, Berlin, 1990. MR 105076322

[Fu] W. FULTON, Intersection Theory, 2nd ed., Ergeb. Math. Grenzgeb. (3) 2, Springer,

Berlin, 1998. MR 164432374

[G] G. VAN DER GEER, Hilbert Modular Surfaces, Ergeb. Math. Grenzgeb. (3) 16,

Springer, Berlin, 1988. MR 0930101 4, 22, 23, 26, 27, 29, 44, 48, 57, 66

[GS1] H. GILLET and C. SOULÉ, Characteristic classes for algebraic vector bundles with Hermitian metric, I, Ann. of Math. (2) 131 (1990), 163-203; II, Ann. of Math.

(2) 131 (1990), 205-238. MR 1038362 ; MR 1043268 7, 16

[GS2] - Arithmetic intersection theory, Inst. Hautes Études Sci. Publ. Math. 72 (1990), 931 - 174. MR 10873947

[Go] E. Z. GOREN, Lectures on Hilbert Modular Varieties and Modular Forms, CRM Monogr. Ser. 14, Amer. Math. Soc., Providence, 2002. MR 1863355 64, 71

[HLR] G. HARDER, R. P. LANGLANDS, and M. RAPOPORT, Algebraische Zyklen auf Hilbert-Blumenthal-Flächen, J. Reine Angew. Math. 366 (1986), 53 - 120. MR 083301366

[H] R. HARTSHORNE, Algebraic Geometry, Grad. Texts in Math. 52, Springer, New York, 1977. MR 046315773

[Ha] W. HAUSMANN, Kurven auf Hilbertschen Modulfächen, Bonner Math. Schriften 123, Univ. Bonn, Mathematisches Institut, Bonn, 1979. MR 05988142

[He] D. A. HEJHAL, The Selberg Trace Formula for PSL(2, $\mathbb{R})$, Vol. 2, Lecture Notes in Math. 1001, Springer, Berlin, 1983. MR 0711197

[Hi] H. HIRONAKA, Resolution of singularities of an algebraic variety over a field of characteristic zero, I, II, Ann. of Math. (2) 79 (1964), 109-203, 205-326.

MR 019918410

[HZ] F. HIRZEBRUCH and D. ZAGIER, Intersection numbers of curves on Hilbert modular surfaces and modular forms of Nebentypus, Invent. Math. 36 (1976), 57 - 113. MR 0453649 2, 6, 29, 37, 48

[I] H. IWANIEC, Introduction to the Spectral Theory of Automorphic Forms, Bibl. Rev. Math. Iberoamericana, Univ. Autónoma de Madrid, Madrid, 1995. MR 1325466

[K] J. KRAMER, An arithmetic theory of Siegel-Jacobi forms, Habilitationsschrift, ETH Zürich, Zürich, 1992. 3

[Ku1] S. S. KUDLA, Algebraic cycles on Shimura varieties of orthogonal type, Duke Math. J. 86 (1997), 39-78. MR 14278457 
[Ku2] Central derivatives of Eisenstein series and height pairings, Ann. of Math. (2) 146 (1997), 545-646. MR 14914482

[Ku3] - "Derivatives of Eisenstein series and arithmetic geometry" in Proceedings of the International Congress of Mathematicians, Vol. II (Beijing, 2002), Higher Ed. Press, Beijing, 2002, 173-183. MR 1957031 2, 3

[Ku4] - Derivatives of Eisenstein series and generating functions for arithmetic cycles, Astérisque 276 (2002), 341 - 368, Séminaire Bourbaki 1999/2000. MR 1886765 2, 3

[Ku5] - Integrals of Borcherds forms, Compositio Math. 137 (2003), 293 - 349. MR 198850155

[Ku6] _ "Special cycles and derivatives of Eisenstein series" in Heegner Points and Rankin L-Series, Math. Sci. Res. Inst. Publ. 49, Cambridge Univ. Press, Cambridge, 2004, 243-270. MR 2083214 2, 3, 7

[KK] S. S. KUDLA and U. KÜHN, Arithmetic intersection numbers of Shimura curves, in preparation. 3, 82

[KR] S. S. KUDLA and M. RAPOPORT, Arithmetic Hirzebruch-Zagier cycles, J. Reine Angew. Math. 515 (1999), 155-244. MR 1717613

[KRY] S. S. KUDLA, M. RAPOPORT, and T. YANG, Derivatives of Eisenstein series and Faltings heights, Compos. Math. 140 (2004), 887-951. MR 2059224 3, 82

[Kü1] U. KÜHN, Generalized arithmetic intersection numbers, J. Reine Angew. Math. 534 (2001), 209-236. MR 1831639 3, 50, 51

[Kü2] _ Über die arithmetischen Selbstschnittzahlen zu Modulkurven und Hilbertschen Modulfächen, Dissertation, Humboldt-Universität zu Berlin, Berlin, 1999. 4, 48, 50, 82

[L] S. LANG, $\mathrm{SL}_{2}(\mathbb{R})$, Addison-Wesley, Reading, Mass., 1975. MR 0430163

[La] A. LANGER, Zero-cycles on Hilbert-Blumenthal surfaces, Duke Math. J. 103 (2000), 131-163. MR 1758242

[MR1] V. MAILLOT and D. ROESSLER, Conjectures sur les dérivées logarithmiques des fonctions L d'Artin aux entiers négatifs, Math. Res. Lett. 9 (2002), 715 - 724. MR 1906073 3, 82

[MR2] - On the periods of motives with complex multiplication and a conjecture of Gross-Deligne, Ann. of Math. (2) 160 (2004), 727-754. MR 21239373

[M] D. MUMFORD, Hirzebruch's proportionality theorem in the noncompact case, Invent. Math. 42 (1977), 239-272. MR 0471627 2, 12

[OT] T. ODA and M. TSUZUKI, Automorphic Green functions associated with the secondary spherical functions, Publ. Res. Inst. Math. Sci. 39 (2003), 451-533.

MR 200118538

[O] A. OGG, "Survey of modular functions of one variable" in Modular Functions of One Variable, I (Antwerp, Belgium, 1972), Lecture Notes in Math. 320, Springer, Berlin, 1973, 1-35. MR 0337785

[OS] K. ONO and C. SKINNER, Fourier coefficients of half-integral weight modular forms modulo $\ell$, Ann. of Math. (2) 147 (1998), 453-470. MR 1626761 3, 61

[P] G. PAPPAS, Arithmetic models for Hilbert modular varieties, Compositio Math. 98 (1995), 43-76. MR 1353285 3, 63, 84 
[R] M. RAPOPORT, Compactifications de l' espace de modules de Hilbert-Blumenthal, Compositio Math. 36 (1978), 255-335. MR 0515050 3, 63, 64, 65

[SABK] C. SOULÉ, D. ABRAMOVICH, J.-F. BURNOL, and J. KRAMER, Lectures on Arakelov Geometry, Cambridge Stud. Adv. Math. 33, Cambridge Univ. Press, Cambridge, 1992. MR 1208731 3, 18, 19

[V] I. VOLLAARD, On the Hilbert-Blumenthal moduli problem, J. Inst. Math. Jussieu 4 (2005), 653-683. MR $217173363,64,70$

[W] T. WEDHORN, personal communication, June 2003. 67

[Z1] D. B. ZAGIER, Modular forms associated to real quadratic fields, Invent. Math. 30 (1975), 1-46. MR 0382174 31, 32, 44

[Z2] Zetafunktionen und quadratische Körper, Hochschultext, Springer, Berlin, 1981. MR 0631688

[Z3] _ " Modular points, modular curves, modular surfaces and modular forms" in Workshop Bonn 1984 (Bonn, 1984), Lecture Notes in Math. 1111, Springer, Berlin, 1985, 225-248. MR 0797423

\section{Bruinier}

Fachbereich Mathematik, Technische Universität Darmstadt, D-64289 Darmstadt, Germany; bruinier@mathematik.tu-darmstadt.de

\section{Burgos Gil}

Facultat de Matemàtiques, Universitat de Barcelona, E-08007 Barcelona, Spain;

burgos@ub.edu

\section{Kühn}

Department Mathematik, Universität Hamburg, D-20146 Hamburg, Germany; kuehn@math.uni-hamburg.de 